Aus der Klinik für Urologie und Kinderurologie

(Chefarzt a. D. Prof. Dr. med. H.-W. Wechsel)

des Klinikums Wilhelmshaven

\title{
Diagnostische und therapeutische Aspekte des Narrow Band Imaging beim \\ Harnblasenkarzinom
}

\author{
INAUGURAL-DISSERTATION \\ zur Erlangung des Doktorgrades \\ der Medizinischen Fakultät der \\ Georg-August-Universität zu Göttingen
}

vorgelegt von

Christoph Zerrenner

aus

Crivitz

Göttingen 2019 
Die vorliegende Dissertation wurde am Klinikum in Wilhelmshaven im Zeitraum von 2014 bis 2019 unter der Betreuung von Prof. Dr. H.-W. Wechsel angefertigt.

Dekan:

Referent:

Ko-Referent:

Drittreferent:
Prof. Dr. rer. nat. H. K. Kroemer

Prof. Dr. med. H.-W. Wechsel

Prof. Dr. med. J. Gaedcke

Prof. Dr. mult. T. Meyer

Datum der mündlichen Prüfung: 06.02.2020 
Hiermit erkläre ich, die Dissertation mit dem Titel „Diagnostische und therapeutische Aspekte des Narrow Band Imaging" eigenständig angefertigt und keine anderen als die von mir angegebenen Quellen und Hilfsmittel verwendet zu haben.

Göttingen, den 


\section{Inhaltsverzeichnis}

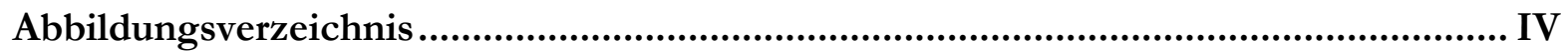

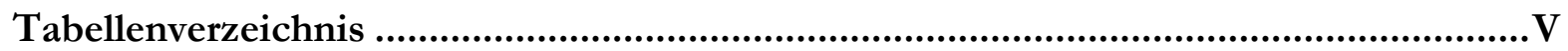

Abkürzungsverzeichnis ............................................................................................... VI

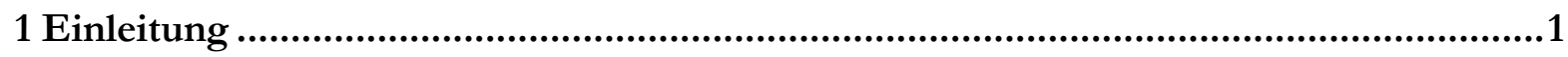

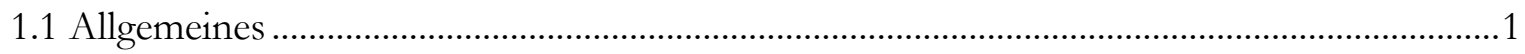

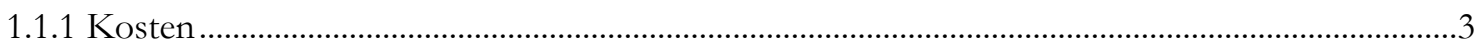

1.2 Definition und Klassifikation .............................................................................................

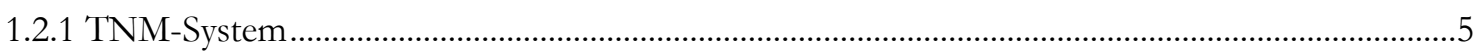

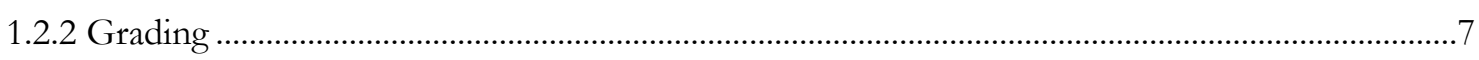

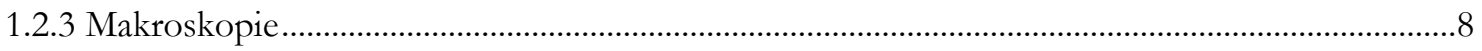

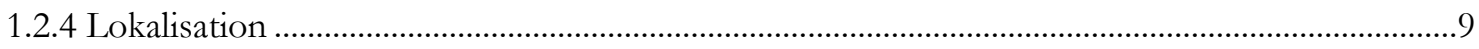

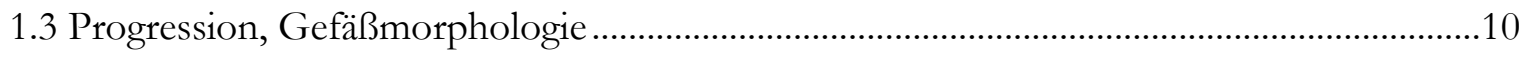

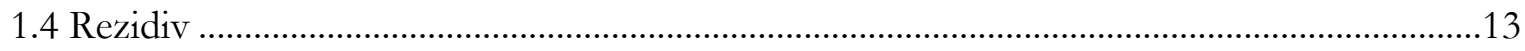

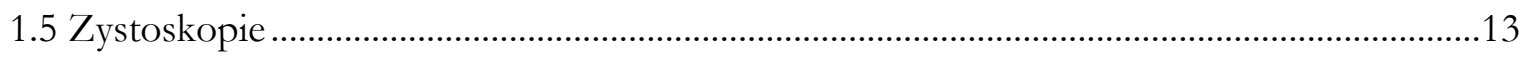

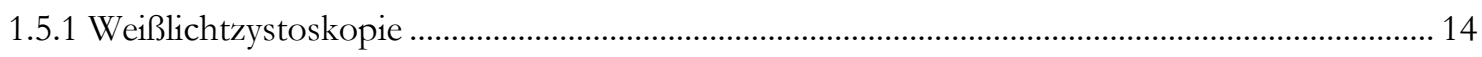

1.5.2 Blaulichtzystoskopie; Photodynamische Diagnostik (PDD) ...................................................... 15

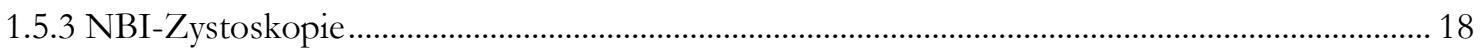

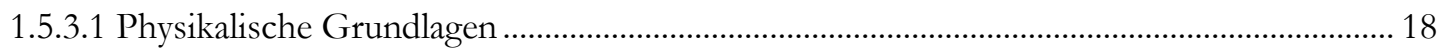

1.5.3.2 Qualitative Beeinträchtigung durch Hexaminolävulinat ....................................................... 20

1.5.3.3 NICE-Kriterien von Iwatate bei kolorektalen Tumoren...................................................... 21

1.6 Primärtherapie des Blasenkarzinoms - die TUR-Blase ............................................................23

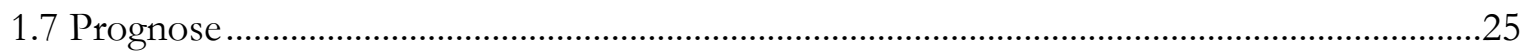

2 Wissenschaftliche Fragestellungen .......................................................................26

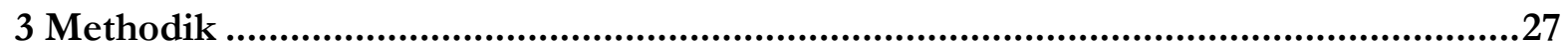

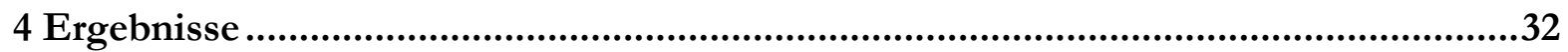




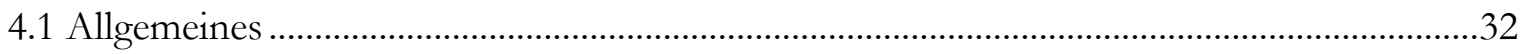

4.2 NBI-Morphologie von Blasenkarzinomen ..............................................................................3

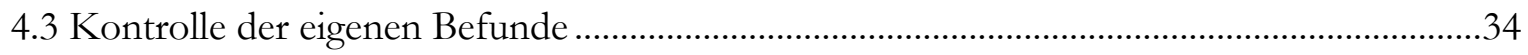

4.4 Untersuchungsergebnisse für Weißlicht und PDD ...................................................................

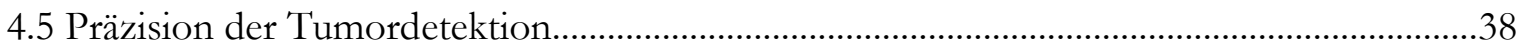

4.6 Präzision der Dignitäts- und Stadienvorhersage .........................................................................39

4.7 Präzision der Ausdehnungsbestimmung des Blasenkarzinoms..............................................40

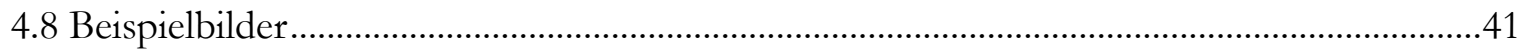

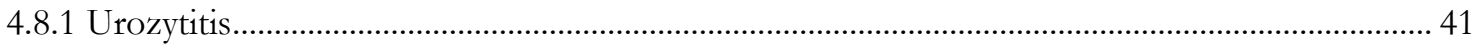

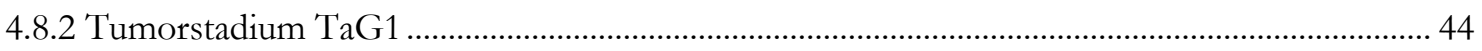



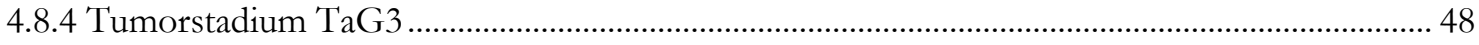

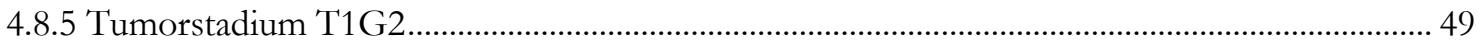



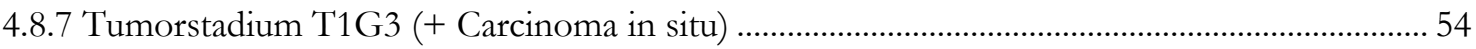

4.8.8 Muskelinvasive Tumoren: T2G2 high grade ............................................................................ 57

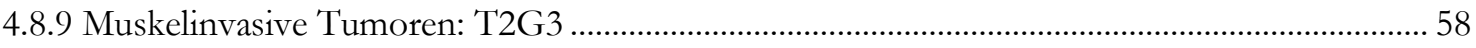

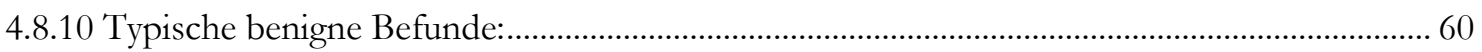

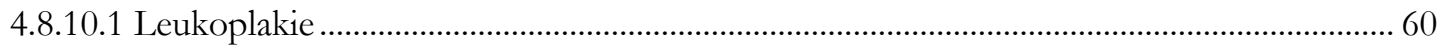

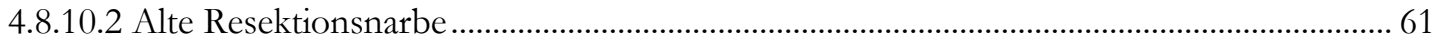

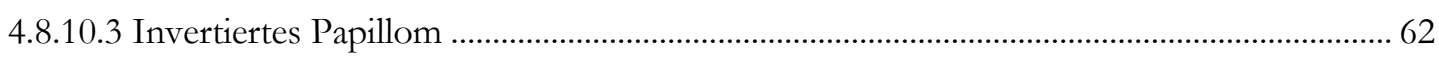

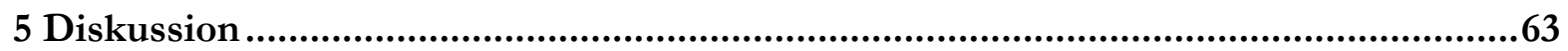

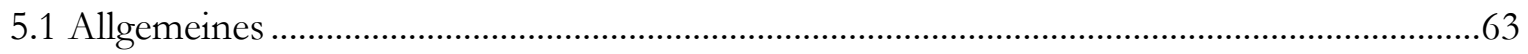

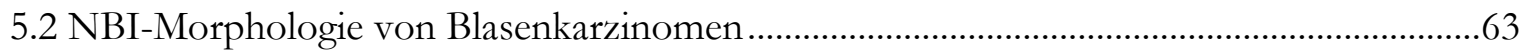

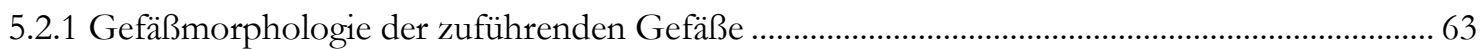

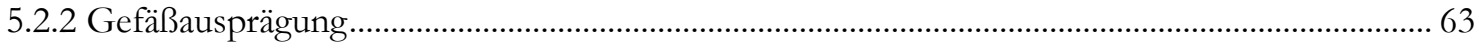

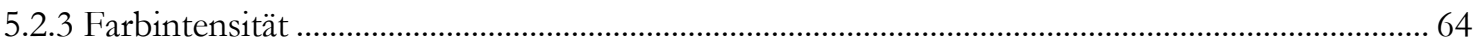

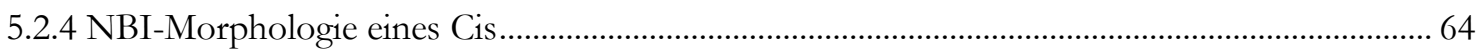


5.3 Vergleich der drei Zystoskopietechniken: Weißlicht, PDD und NBI-Tumordetektion ....65

5.3.1 Vorhersage der Histologie

5.4. Präzision der Ausdehnungsbestimmung des Blasenkarzinoms...............................................69

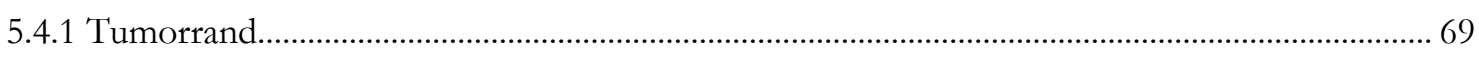

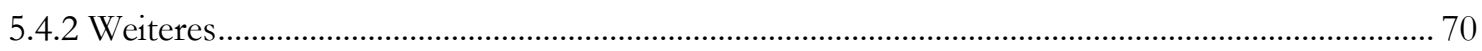

5.5 Diskussion von Material und Methoden .................................................................................

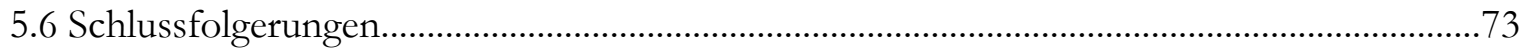

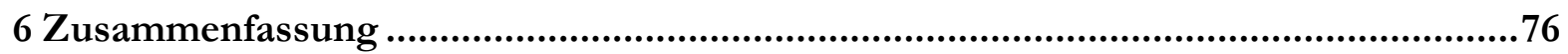

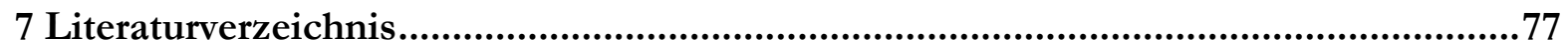




\section{Abbildungsverzeichnis}

Abbildung 1 Inzidenz der Neuerkrankungen der Harnblase.

Abbildung 2: Schematische Darstellung der Tumorstadien......................................................... 6

Abbildung 3: Papilläres Blasenkarzinom in NBI .................................................................... 8

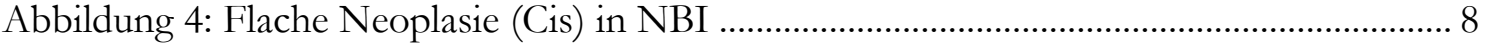

Abbildung 5: Schematische Darstellung der Entwicklung zum Blasenkarzinom ................... 11

Abbildung 6: Fiberoskop zur flexiblen Zystoskopie: Typ CYF-VH ........................................ 14

Abbildung 7: Starres Resektoskop mit beweglicher Resektionsschlinge................................... 14

Abbildung 8: Schematische Darstellung einer Zystoskopie........................................................ 15

Abbildung 9: Prinzip der Fluoreszenzzystoskopie ...................................................................... 17

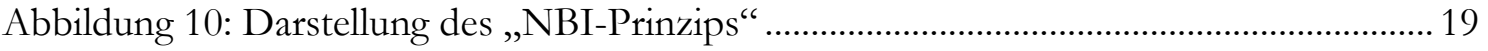

Abbildung 11: Vergleich Blasenschleimhaut in Weißlicht mit NBI-Technik........................... 20

Abbildung 12: NICE-Klassifikation in der Gastroenterologie................................................. 22

Abbildung 13: Die drei verfolgten Ziele einer TUR der Blase .................................................. 24

Abbildung 14: Ablaufschema der Blasenkarzinom-/Hämaturieabklärung............................... 28

Abbildung 15: Schema zur Technik der differenzierenden TUR-Blase .................................... 30

Abbildung 16: SOP zum Ablauf und Aufbau der TUR-Blase in den verschiedenen

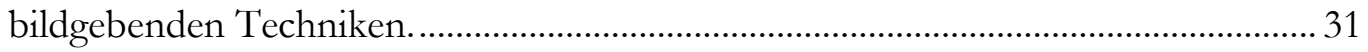

Abbildung 17: Schematische Zeichnungen der Morphologie einer Urozystitis, eines low gradeTumors und eines high grade-Tumors. (von links nach rechts) 


\section{Tabellenverzeichnis}

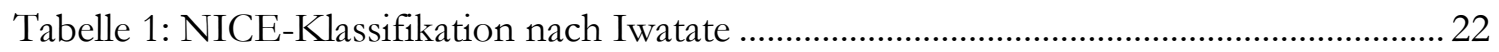

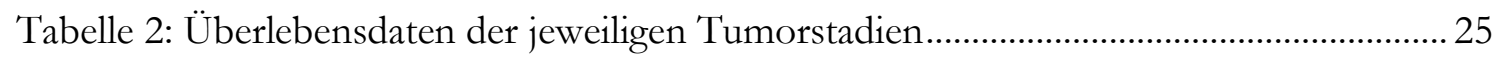

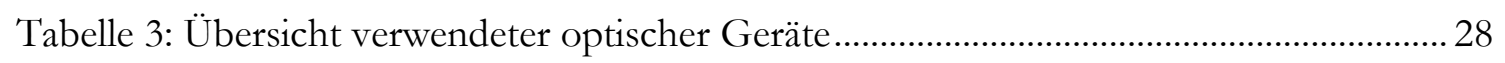

Tabelle 4: Statistische Übersicht zu den Residualtumorraten nach TUR-B ............................. 29

Tabelle 5: Schema für die Bewertung von Blasentumoren in der NBI-Zystoskopie............. 33

Tabelle 6: Ergebnisse hinsichtlich der NBI-Prognose und der Tumorrandbestimmung..... 34

Tabelle 7: Ergebnisse Weißlicht- und PDD-Resektion in Tumordetektion, Unterscheidung benigner Befunde und Bestimmung der Tumorgrenzen ............................................... 37

Tabelle 8: Vorgeschlagenes Schema für eine Anschlussstudie zur Beurteilung der bildgebenden Techniken und ihrer optimalen Sequenz. 


\section{Abkürzungsverzeichnis}

\begin{tabular}{|c|c|}
\hline BCG & Bacillus Calmette-Guérin \\
\hline $\mathrm{BN}$ & bösartige Neubildungen \\
\hline Cis & Carcinoma in situ \\
\hline CK20 & Cytokeratin 20 \\
\hline CMCI & Zytostatikum-Mikrosphären-Karzinom-Infusion \\
\hline DGU & Deutsche Gesellschaft für Urologie \\
\hline DRG & disease-related groups \\
\hline GEKID & Gesellschaft der epidemiologischen Krebsregister in Deutschland \\
\hline $\mathrm{HD} / \mathrm{UHD}$ & bigh definition/ultra high definition \\
\hline ICD-10 & International Statistical Classification of Diseases and Related Health Problems \\
\hline NBI & Narrow Band Imaging \\
\hline NICE & NBI International Colorectal Endoscopy \\
\hline NMIBC & non-muscle-invasive bladder cancer \\
\hline NAT & N-Acetyltransferase \\
\hline P53 & Protein 53 (Tumorsuppressorprotein) \\
\hline PDD & photodynamische Diagnostik \\
\hline PD-ECGF & platelet-derived endothelial cell growth factor \\
\hline PUNLMP & $\begin{array}{l}\text { papillary urothelial neoplasia of low malignant potential (Papilläre } \\
\text { Urothelneoplasie mit niedrigmalignem Potential) }\end{array}$ \\
\hline RFS & recurrence-free survival \\
\hline SOP & standard operating procedure (Standardvorgehen; bspw. im OP-Saal) \\
\hline TP53 & Tumorsuppressorgen 53 \\
\hline TUR-B & Transurethrale Resektion der Blase \\
\hline VEGF & vascular endothelial growth factor \\
\hline Z. n. & Zustand nach (beispielsweise einer Operation) \\
\hline
\end{tabular}




\section{Einleitung}

\subsection{Allgemeines}

Das Harnblasenkarzinom gehört mit einer Inzidenz von circa 15.000 Neuerkrankungen (lediglich ICD-10: C67; ausgenommen ICD-10: D41.4 und D09.0) pro Jahr zu den häufigsten bösartigen Tumoren und ist somit der zweithäufigste maligne Urogenitaltumor. Die Inzidenz des Harnblasentumors (mit D41.4und D09.0) liegt mit ca. 27.000 Neuerkrankungen noch weitaus höher. Sie korreliert zudem mit dem Alter (Gekid.de 2011-2014).

Männer sind hierbei mit durchschnittlich knapp drei Viertel der Fälle deutlich häufiger betroffen als Frauen. Jeder 25. westliche Mann und jede 80. Frau wird einmal im Leben ein Blasenkarzinom diagnostiziert bekommen (Ploeg et al. 2009). Etwa zwei Drittel der Fälle sind dabei nicht muskelinvasiv. Die Inzidenz ist in den letzten Jahren nahezu stabil (Abbildung 1), in Europa und den USA sogar leicht abnehmend. Dies ist wahrscheinlich auf die veränderten Rauchgewohnheiten und einen besseren Berufsschutz zurückzuführen, da alle anderen Risikofaktoren stabil sind. Entsprechend ist die Inzidenz in Entwicklungsländern entgegengesetzt (Ploeg et al. 2009).

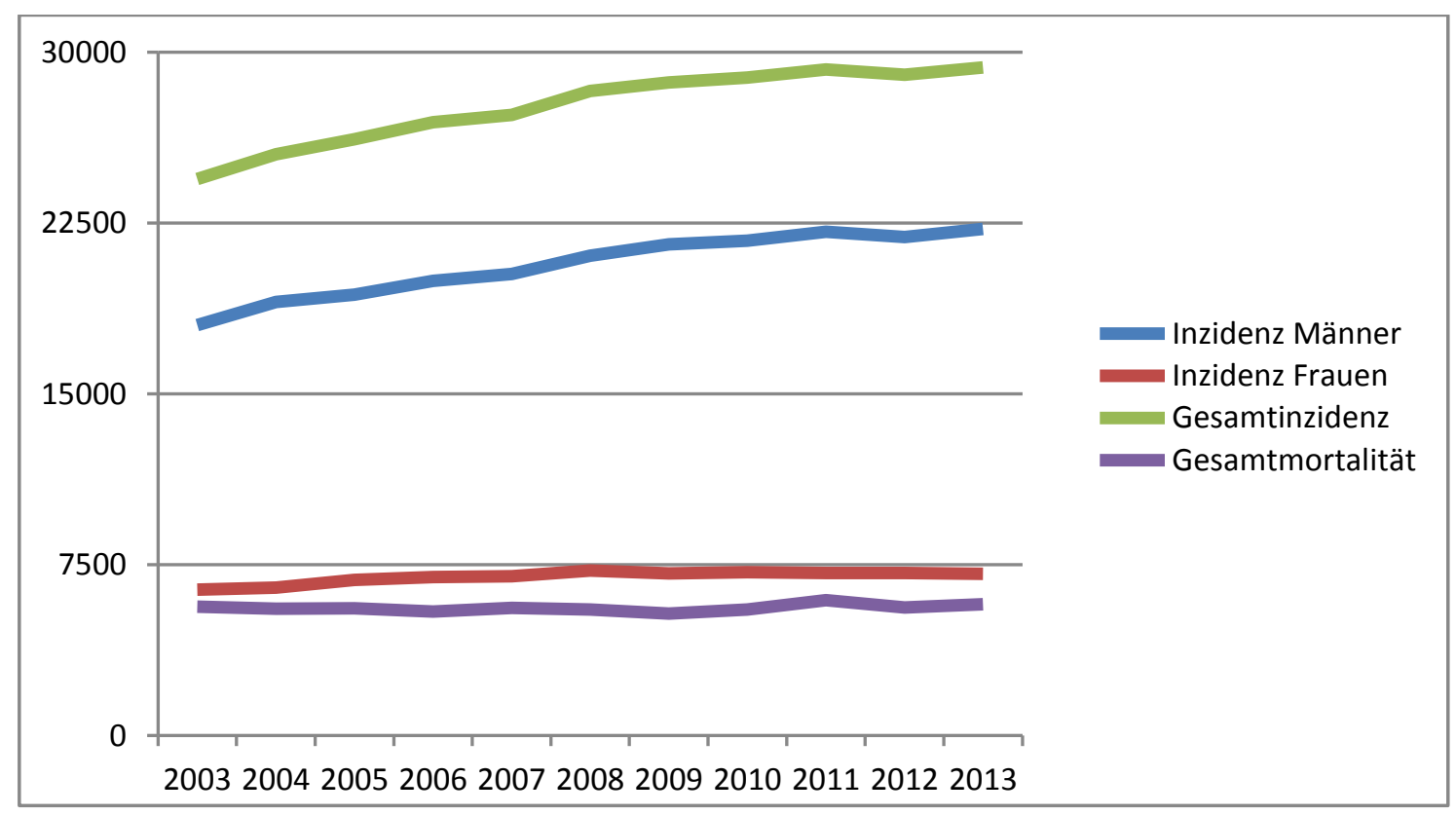

Abbildung 1 Inzidenz der Neuerkrankungen der Harnblase (ICD-10: C67+D09.0+D41.4) nach Geschlecht, sowie Gesamtmortalität des Blasenkarzinoms (ICD-10: C67) in Deutschland (modifiziert nach Meyer 2016) 
Aufgrund der hohen Rezidivrate des Blasenkarzinoms von über 70 Prozent (Chamie et al. 2013) und des damit verbundenen Nachsorgeaufwands entstehen erhebliche Folgekosten. Somit ist die Therapie des Blasenkarzinoms insgesamt relativ teuer (s. auch 1.1.1 Kosten) (Racioppi et al. 2007).

Der Verdacht auf ein Harnblasenkarzinom ergibt sich häufig aufgrund einer Makrohämaturie oder als Zufallsbefund vor allem in der Abklärung einer Mikrohämaturie (Manski 2017).

Bei Verdacht auf ein Harnblasenkarzinom können verschiedene, in ihrer Aussagekraft jedoch stark eingeschränkte nichtinvasive Diagnostika eingesetzt werden (Khadra et al. 2000):

- Zytologie des nativen Urins, bei hochdifferenzierten Tumoren (Frühstadium, s. u. Grading) jedoch nur in ca. $20 \%$ bis $50 \%$ der Fälle richtig positiv. Dafür erreicht die Zytologie mit ca. $95 \%$ eine hohe Spezifität (Turco et al. 2011).

- Sonographie des Urogenitaltraktes mit einer ebenfalls eingeschränkten Sensitivität und Spezifität (20\% für Tumoren kleiner als 5 mm) (Nicolau et al. 2011)

- Untersuchung des Urins mittels verschiedener industriell angebotener Tests, z. B. NMP 22. Alle diese Testverfahren zeigen eine nicht ausreichende Sensitivität und Spezifität und werden somit in der Standarddiagnostik nicht von den Leitlinien gestützt ((EAU et al. ; Leitlinienprogramm Onkologie (Deutsche Krebsgesellschaft 2016)

- Zum Ausschluss eines Befalls des oberen Harntraktes erfolgt bei fortgeschrittenen Stadien (s. 1.2.1 TNM-System) oder Befall des Trigonums eine radiologische Abklärung vorwiegend in Form einer Computertomographie mit Abflusstopographie

Zentrales Diagnostikum ist die per se invasive Urethrozystoskopie mit ggf. Probenentnahme zur Dignitätssicherung oder die gleichzeitig in vielen Fällen kurative transurethrale Resektion (Hautmann und Huland 2006; Schmelz et al. 2007). Die Urethrozystoskopie ist damit der „Referenzstandard“.

- Im Rahmen der meistens ambulant durchgeführten Urethrozystoskopie wird der Blasentumorverdacht makroskopisch erhärtet, im weiteren Verlauf folgt die TUR-Blase mit verschiedenen Zielsetzungen (s. u. 1.6 Primärtherapie des Blasenkarzinoms). Ein sicherer Ausschluss eines malignen Wachstums bereits während der Urethrozystoskopie ist bisher nicht möglich, wäre aber zur Minimierung der Invasivität, Patientenbelastung und Kostenreduktion durch Einsparung weiterer invasiver Diagnostika und Therapien wünschenswert. 
- Bei oberflächlichen Tumoren können anhand des Gewebematerials die histologische Sicherung der Diagnose und eine exakte Stadieneinteilung erfolgen. Gelingt eine vollständige Resektion des Tumors, ist diese gleichzeitig in der Regel die Kuration. In diesem Zusammenhang ist die exakte Definition der Tumorgrenzen essentiell. Dieses wurde in den letzten Jahrzehnten z. B. durch Randproben, Proben vom Tumorgrund (mit der Frage der Infiltrationstiefe) und ggf. Nachresektionen erreicht (Bichler et al. 1998c).

- Bei fortgeschrittenen Tumoren kann anhand des histologischen Befundes und der radiologischen Ausbreitungsdiagnostik die weitere Therapie geplant werden. Hier wäre eine zystoskopische Zusatzinformation über die mögliche Infiltrationstiefe bzw. die Ausbreitung des Blasenkarzinoms wünschenswert.

Eine Verbesserung der bisher gepflegten Weißlichtzystoskopie wäre somit aus medizinischen, aber auch aus ökonomischen Gründen erstrebenswert.

\subsubsection{Kosten}

Das Harnblasenkarzinom ist prozentual die neunthäufigste Todesursache (ca. 52.000 Tote in der EU/Jahr). Da der Tumor zumeist in niedrigeren Stadien diagnostiziert wird, ist die Lebenserwartung gut und die Prävalenz somit erhöht. Hinzu kommt eine hohe Rezidivrate. Hiermit ist eine engmaschige Nachsorge verbunden. Die genannten Faktoren gehen mit einer deutlichen Kostenzunahme einher (ca. 760 Millionen Euro/Jahr in Deutschland), die Kostenzunahme ist stark steigend (GBE-Bund.de 2017).

In der europäischen Union werden jährlich etwa 5 Milliarden Euro für Diagnostik und Behandlung des Blasenkarzinoms ausgegeben (Kramer et al. 2015), damit sind drei Prozent aller onkologischen Kosten auf das Blasenkarzinom zurückzuführen (Leal et al. 2016). In den USA verursacht ein Blasenkrebspatient zwischen 96.000 und 187.000 Dollar Kosten (Botteman et al. 2003). Die Gesamtkosten belaufen sich auf insgesamt 4,5 Milliarden Dollar pro Jahr (Yeung et al. 2014). Circa $60 \%$ der Kosten entfallen dabei auf die Behandlung und ca. $30 \%$ auf die Komplikationen. Ein Patient, der in der Tumorprogression verstirbt, verursacht in etwa $20 \%$ weniger Kosten als ein Patient, der ohne Progression überlebt (Avritscher et al. 2006). 
Eine Option zur Kostenersparnis ergibt sich aus den vermuteten Möglichkeiten des NBIVerfahrens durch präzisere Grenzbestimmung des Tumors und eine verbesserte Tumordetektion mit der vermuteten Reduktion von Folgeeingriffen. 


\subsection{Definition und Klassifikation}

Das Harnblasenkarzinom ist eine von den Urothelzellen ausgehende bösartige Tumorerkrankung der Harnblase. Eingeteilt wird nach der TNM-Klassifikation (siehe unten: 1.2.1 TNM-System).

Andere in der Harnblase vorkommende Tumorformen sind selten, z. B. Adenokarzinome, Sarkome oder das Plattenepithelkarzinom, welches vorrangig bei Patienten mit chronischen Infektionen oder Schistosomiasis auftritt.

Klinisch-therapeutisch ist die Unterscheidung zwischen nicht-muskelinvasiven und muskelinvasiven Karzinomen wesentlich. Nicht-muskelinvasive Harnblasenkarzinome lassen sich ferner einteilen in Tumoren, welche die Lamina propria infiltrieren (Stadium T1) und solche, die dies nicht tun. Letztere gehören zum Stadium Ta und können zu $95 \%$ mit einer TUR-B geheilt werden (Bichler et al. 1998a). Muskelinvasive Harnblasenkarzinome haben generell eine schlechtere Prognose und erfordern in der Regel die radikalchirurgische Entfernung der Harnblase. Die tumorfreie 5-Jahres-Überlebensrate liegt bei $63 \%$ mit T2- und $50 \%$ mit T3Tumoren nach radikalchirurgischer Intervention (Shariat et al. 2006; Yuh et al. 2015).

\subsubsection{TNM-System}

Die Stadieneinteilung erfolgt klinisch relativ ungenau, z. B. nach Palpation des Tumors (mindestens T3) oder nach radiologischer Bildgebung, wesentlich genauer anhand der Histologie.

Mit dem T-Stadium wird die Infiltrationstiefe beschrieben. Abbildung 2 zeigt die verschiedenen Tumorstadien. Ta-Tumoren sind papilläre Tumoren. Hiervon gibt es mehrere Unterformen. Es wird zwischen seltenen gutartigen (beispielsweise das Papillom) und bösartigen Läsionen wie dem Urothelkarzinom unterschieden (Moch et al. 2016). In der Dignität liegt die PUNLMP zwischen dem Papillom und dem papillären Urothelkarzinom. Diese entarten im Prinzip nie bis zum NMIBC, haben aber eine hohe Rezidivrate (Al Bashir et al. 2014; Campbell et al. 2004). Das Wachstum ist auch beim Rezidiv meist auf das Urothel begrenzt. T1-Tumoren wachsen bereits infiltrativ in die subepitheliale Schicht. T2-Tumoren infiltrieren den Blasenmuskel (T2a: Muscularis propria innere Schicht; T2b: Muscularis propria äußere Schicht). Die Stadien T3, mit mikroskopischer (T3a) und makroskopischer (T3b) perivesicaler Tumorinfiltration sowie T4, mit Infiltration der Prostata, Vagina, Uterus (T4a) oder auch der Becken- oder Bauchwand (T4b) gelten als lokal fortgeschrittene Blasenkarzinome. 
Eine Besonderheit stellt das Carcinoma in situ (Cis) dar. Es bildet keine Metastasen und respektiert die Basallamina, es ist also nicht infiltrativ. Jedoch hat das Cis eine schlechte Differenzierung (immer high grade) und kann sich zu einem invasiven Karzinom entwickeln. Es ist assoziiert mit einer höheren Progressions- und Rezidivrate (Lamm et al. 1998; Stanisic et al. 1987; Utz et al. 1970). Da es eine flache, auf das Urothel beschränkte Läsion ist, gestaltet sich die zystoskopische Diagnosestellung im Verhältnis zu den papillären oder soliden Tumoren erschwert.

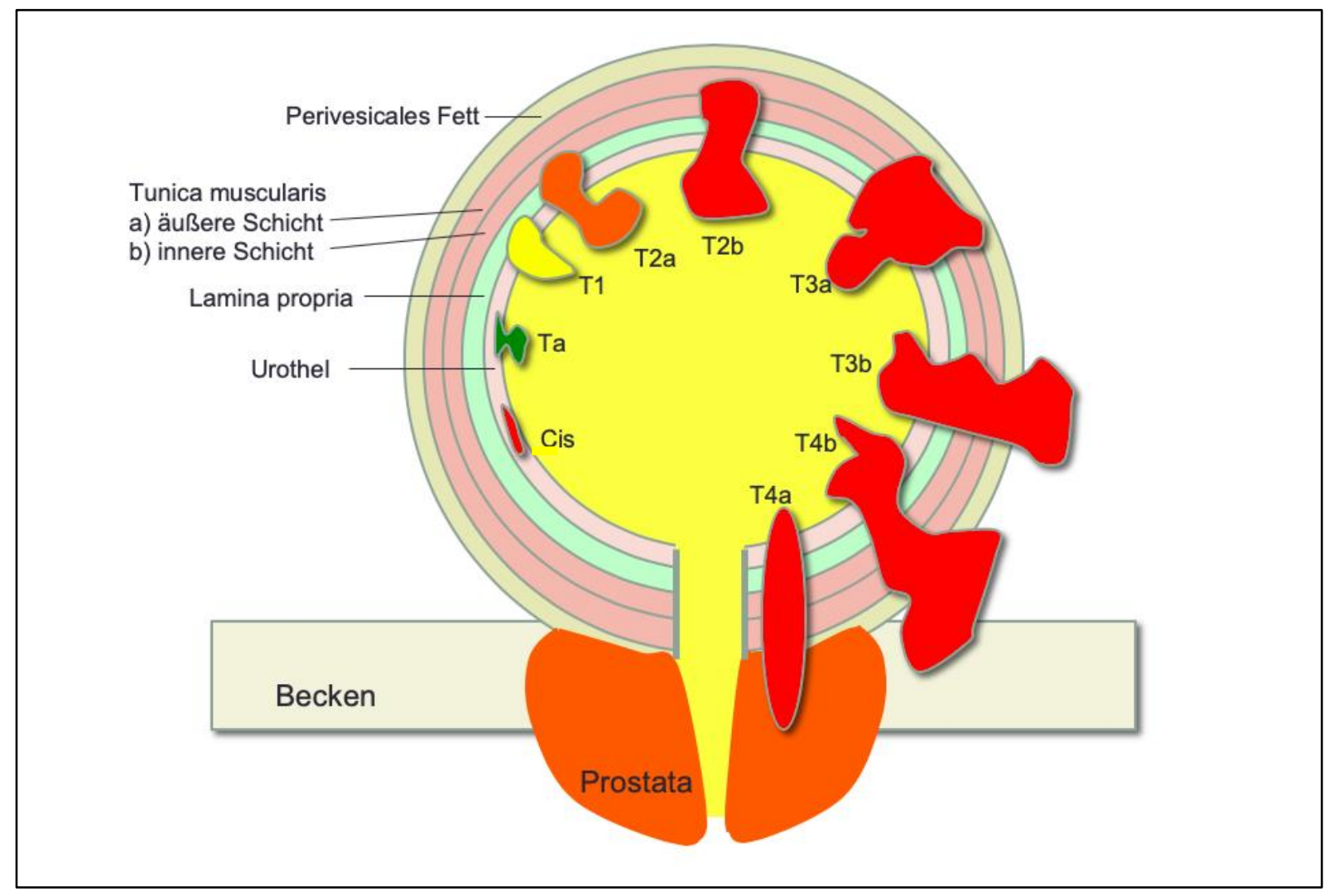

Abbildung 2: Schematische Darstellung der Tumorstadien

Das N- bzw. M-Stadium wird in erster Linie radiologisch definiert, das N-Stadium bei radikalchirurgischem Eingriff histologisch präzisiert.

Der Befall eines Lymphknotens entspricht dem Stadium N1, multiple Lymphknotenmetastasen entsprechen dem Stadium N2, das Stadium N3 bedeutet multiple Lymphknotenmetastasen entlang der Aa. iliacae communes. Das M-Stadium spiegelt die Fernmetastasierung wider. 


\subsubsection{Grading}

Die Entdifferenzierung der Tumorzelle von der normalen Urothelzelle wird durch das Grading beschrieben. Je geringer die Ausprägung der Aufreihung von Basalzellen auf der Bindegewebsschicht, des sogenannten palisading, und je mehr Kernatypien vorhanden sind, desto geringer ist der Differenzierungsgrad des Tumors und desto höher sein malignes Potential.

2004 definierte die WHO im Gegensatz zur Klassifikation von 1973 erstmals lediglich Unterschiede zwischen bigh grade- und low grade-Tumoren. Die Klassifikation unterschied zur besseren Reproduzierbarkeit und wegen der gleichlautenden Verwendung für die Urinzytologie (Moch et al. 2016) in high- und low grade-Tumoren. Es kam zu einer Überschneidung der histologischen Angaben, sodass bei G2-Tumoren zusätzlich zwei Substufen in Form von low bzw. high grade unterschieden wurden (Helpap und Kollermann 2000). Die neue Blasenkarzinomleitlinie empfiehlt neben der Unterscheidung von high und low grade weiterhin die zusätzliche Angabe von G1; G2 oder G3 nach alter WHO-Klassifikation, da dies gerade bei T1Tumoren von prognostischer Bedeutung ist.

Zudem unterscheidet die WHO-Klassifikation von 2016 vor allem invasive Tumoren (ab T1) histologisch noch genauer. Grund hierfür ist ebenfalls die unterschiedliche Prognose- (bspw. 3fach höheres Progressionsrisiko des netzartigen Infiltrationstyps gegenüber dem plumpen Infiltrationstyps und Therapieansätze der verschiedenen Infiltrationsmuster (Wittekind und Meyer 2010). Die Datenlage ist insbesondere bei mischdifferenzierten Tumoren noch unzureichend. In der exakteren histologischen Einteilung liegt ein großes Entwicklungspotential hinsichtlich einer Therapieanpassung und -optimierung. 


\subsubsection{Makroskopie}

Neben der Histologie und dem Grading erfolgt auch eine rein zystoskopisch-makroskopische Unterteilung des Blasenkarzinoms. Hier spielt neben der Frage nach dem singulären bzw. multilokulären Auftreten des Tumors ebenso die Tumorgröße eine Rolle. Multilokulärer Befall der Harnblase ist wie die zunehmende Tumorgröße $(>3 \mathrm{~cm}$ ) prognostisch ungünstig (Eble et al. 2004).

Weiterhin werden Blasentumoren makroskopisch in flache und papilläre Neoplasien unterteilt. Mit Letztgenanntem ist eine hohe Rezidiv- und Progressionsrate assoziiert. Das Dilemma ist eine zystoskopisch deutlich erschwerte Detektion (Jocham et al. 2008). Abbildung 3 und Abbildung 4 zeigen den zystoskopischen Unterschied zwischen einem gut sichtbaren papillären Tumor und einer flachen Neoplasie in NBI-Technik.

Invasive Tumoren haben makroskopisch häufig einen soliden Aspekt. Sie wachsen zumeist -wie die papillären Tumoren- in das Blasenlumen. Die Detektion ist somit leichter als beim Cis, jedoch ist der Tumorrand schwieriger festzulegen als bei papillären Tumoren.

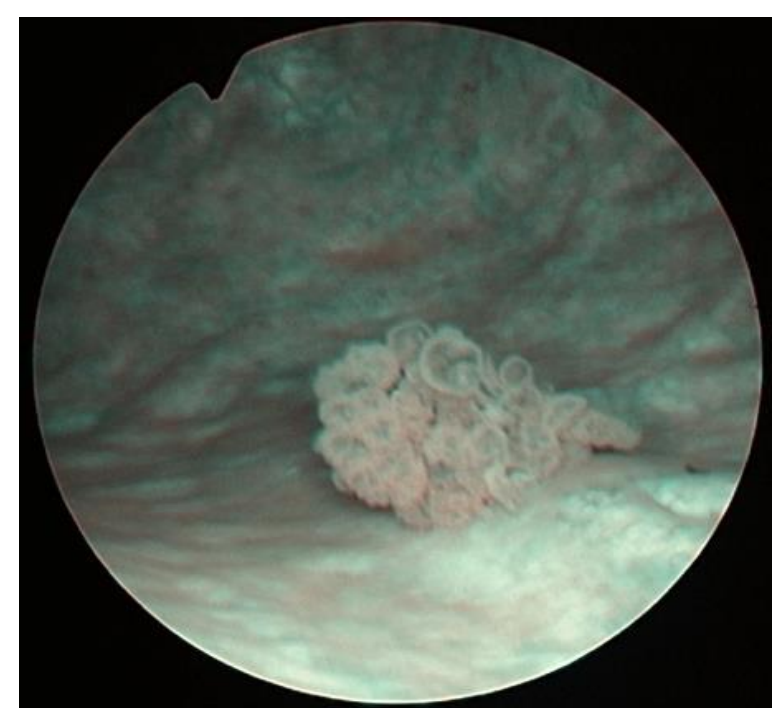

Abbildung 3: Papilläres Blasenkarzinom in NBI

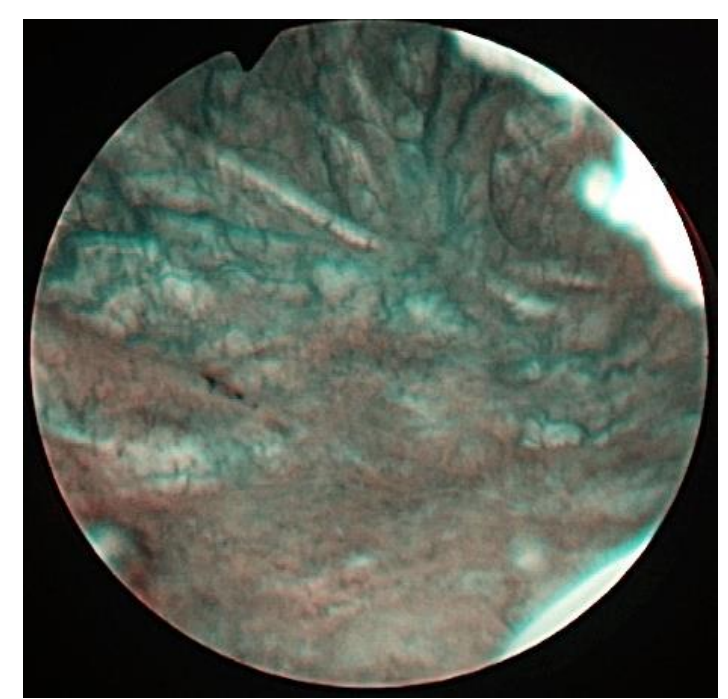

Abbildung 4: Flache Neoplasie (Cis) in NBI 


\subsubsection{Lokalisation}

Makroskopisch ist in circa 70\% der Fälle die Seiten- oder Hinterwand vom Blasenkarzinom befallen, nur in $10 \%$ die Vorderwand. Die Ursachen für diese Inkongruenz sind letztlich nicht geklärt. Eine iatrogene Komponente wird noch untersucht, eine Studie von Adiyat et al. (2010) lässt zumindest darauf schließen.

Liegt ein Tumor im Trigonumbereich, sollte eine Untersuchung des oberen Harntraktes (bestenfalls eine CT-Urographie) erfolgen, da synchrone Urothelkarzinome mit $7 \%$ dann in etwa doppelt so häufig vorkommen (Goessl et al. 1997; Herranz-Amo et al. 1999; Palou et al. 2005). 


\subsection{Progression, Gefäßmorphologie}

Die Progressionstendenz des Harnblasenkarzinoms wird vorrangig über eine Veränderung der Expression des Tumorsuppressorgens TP53 erklärt, die wiederum über chromosomale Veränderungen (hier: Chromosom 17) nach exogener oder endogener Schädigung entsteht (Adiyat et al. 2010). Durch Mutationen des p53 Tumorsuppressorproteins kommt es zu einer Häufung des Proteins bei infiltrativen, schlecht differenzierten Tumoren.

Dahingegen ist der Verlust des langen Arms von Chromosom 9 nicht nur bei flachen Neoplasien, sondern auch bei papillären Neoplasien ( $\mathrm{Ta}$ ) nachweisbar. Der Verlust einer Heterozygotie für das Chromosom 9 wird als einer der entscheidenden Mechanismen bei der Entstehung eines papillären Tumors angesehen (Droller 1998; Sarkis et al. 1994).

Des Weiteren wurden bei Urothelkarzinomen eine erhöhte VEGF-Expression und MMP-9Expression festgestellt. Die Überexpression dieser Tumorpromotoren ist ein wesentlicher Faktor der Tumorangiogenese und proportional zur Invasionstiefe und Aggressivität (Donmez et al. 2009). Es resultiert die Erwartung einer vermehrten Gefäßeinsprossung bei zunehmend aggressiveren Tumoren. Zudem besteht eine Assoziation mit der Metastasenbildung (Brandau und Bohle 2001). Die Beeinflussung der Angiogenese ist ein Gegenstand moderner Tumortherapien (Cao et al. 2011).

Auch PD-ECGF (platelet-derived endothelial cell growth factor) ist ein Angiogenesefaktor, der vor allem in bigh grade-Tumoren, bei physkalischem oder chemischen Stress vermehrt exprimiert wird.

FGFR3 (fibroblast growth factor receptor-3) Genmutationen treten vor allem in NMIBC auf. Sie treten außer bei T1 Blasenkarzinomen nicht simultan mit p53-Mutationen auf (Apolo et al. 2015). 


\section{Blasenkarzinom: Entwicklung}

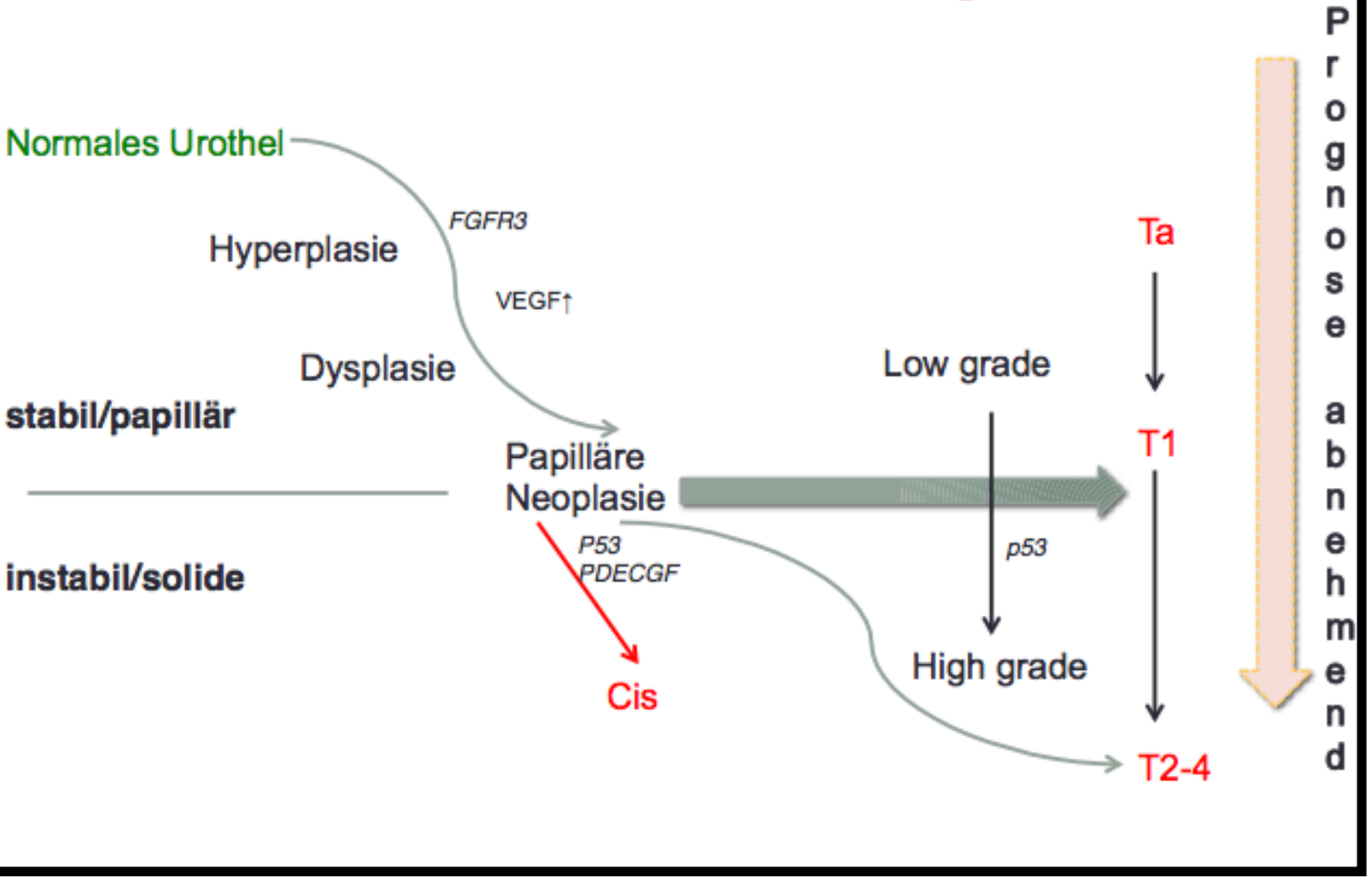

Abbildung 5: Schematische Darstellung der Entwicklung zum Blasenkarzinom (eigene Bildersammlung; Erläuterungen siehe Fließtext)

Die Abbildung 5 stellt ein Modell der wesentlichen genomischen Veränderungen in der Karzinogenese des Harnblasenkarzinoms dar. Über die Transformation erlangen die pathologisch veränderten Zellen einen Wachstumsvorteil gegenüber dem gesunden Urothel, da die Kontrollpunkte der Zellproliferation inaktiviert werden (Deppert 1999). Während sich papilläre Formen über eine Hochregulierung von VEGF sowie Cyclin D Amplifikation und den Verlust der Heterozygotie von Chromosom 9 entwickeln, kommt es bei Cis oder invasiven Blasenkarzinomen $\mathrm{zu}$ einer p53 Inaktivierung und PDECGF Expression. Aus der unterschiedlichen Expression angiogenetischer Faktoren ist ein unterschiedliches Vaskularisierungsmuster in beiden potentiellen Entwicklungsreihen des Tumors zu erwarten. Die Progressions- und Rezidivtendenz steigt mit zunehmender Expression von p53 und CK20, eine prospektive Analyse steht hierzu noch aus. 
Die Gefäßmorphologie für prädiktive Aussagen zu benutzen wurde bereits 1993 von Folberg et al. (1993) bei Ziliarkörpermelanomen untersucht. Neben histologisch sichtbaren Unterschieden wie einer erhöhten Leckage der Gefäße oder einer unterbrochenen Basalmembran gibt es auch makroskopische Unterschiede. Mehrere Untersuchungen zeigten, dass Tumorgefäße, verglichen mit physiologischen oder gutartig veränderten Gefäßen, einen größeren Durchmesser haben (Abramsson et al. 2003; Dennie et al. 1998). Weiterhin zeigt sich die Verzweigung der Gefäße oftmals nicht hierarchisch, sondern stark irregulär, teilweise auch ohne Bezug zueinander (Dennie et al. 1998). Da es starke Unterschiede in der Angiogenese menschlicher Tumoren gibt, kann es auch Unterschiede und Gemeinsamkeiten in der makroskopischen Gefäßdarstellung der jeweiligen Tumorentität geben. Dies wurde für Blasenkarzinome noch in keiner der vorliegenden Studien untersucht.

Eine makroskopisch-zystoskopische Darstellung der postulierten Gefäßmorphologie wäre letztlich ein möglicher weiterer Prognosefaktor für die Gesamtbeurteilung des Blasenkarzinoms. 


\subsection{Rezidiv}

Das Blasenkarzinom hat eine hohe Progressions- und Rezidivtendenz. $70 \%$ der Blasentumoren rezidivieren nach erfolgreicher Initialtherapie (Sylvester et al. 2006). Eine entscheidende Bedeutung kommt der Beurteilung des Schnittrandes zu. Bei positivem Resektionsrand ist das Rezidivrisiko deutlich erhöht (Gakis et al. 2011; Gakis et al. 2013). Weitere Risikofaktoren sind Mulitlokularität, begleitendes Cis, Tumorgröße, Rezidivrate in der Anamnese und das Grading.

\subsection{Zystoskopie}

Die (Urethro)zystoskopie (kýste „die Blase“; skopé „die Schau“) ist zentrales Element in der Diagnostik eines vermuteten Blasenkarzinoms. Abbildung 8 demonstriert die Zystoskopie bei Mann und Frau. Sie ist einfach anzuwenden und besitzt eine hohe Detektionsrate (Cina et al. 2001; Hautmann und Huland 2006) und ist damit der „Referenzstandard“. Weitere diagnostische Maßnahmen können hinzugenommen werden, ersetzen aber letztlich nicht die Urethrozystoskopie. Den Grundstein für die heutige Zystoskopie legte Maximlian Nitze bereits Ende des 19. Jahrhunderts (Schultheiss et al. 1999). In den letzten Jahrzehnten wurde die Technik nur noch wenig verbessert.

Verwendet werden entweder starre oder flexible Geräte (s. Abbildung 6 und Abbildung 7), die über ein Kaltlichtkabel ausreichend Licht in die Harnröhre bzw. Harnblase projizieren, um mit einer $0^{\circ}$ - bzw. $12^{\circ}$-Optik (sog. Geradeausoptik) bzw. einer angeschrägten $70^{\circ}$-Optik (starre Optik) die gesamte Harnröhre und alle Anteile der Harnblase zu inspizieren. Mit dem flexiblen Zystoskop ist das Sichtfeld sogar bis zu $120^{\circ}$ weit. Zudem ist das flexible Zystoskop aufgrund des geringeren Außendurchmessers und seiner Flexibilität bei gleicher Detektionsrate (Walker et al. 1993) für den Patienten angenehmer (Denholm et al. 1990; Michaud et al. 1999). Der Sterilisationsaufwand ist aber bei geringerer Lebensdauer erhöht. Starres und flexibles Zystoskop bieten eine optische Vergrößerung. Zusätzlich kann eine weitere Vergrößerung mittels Bildschirmprojektion elektronisch erfolgen. Standard ist die Weißlichtzystoskopie. 


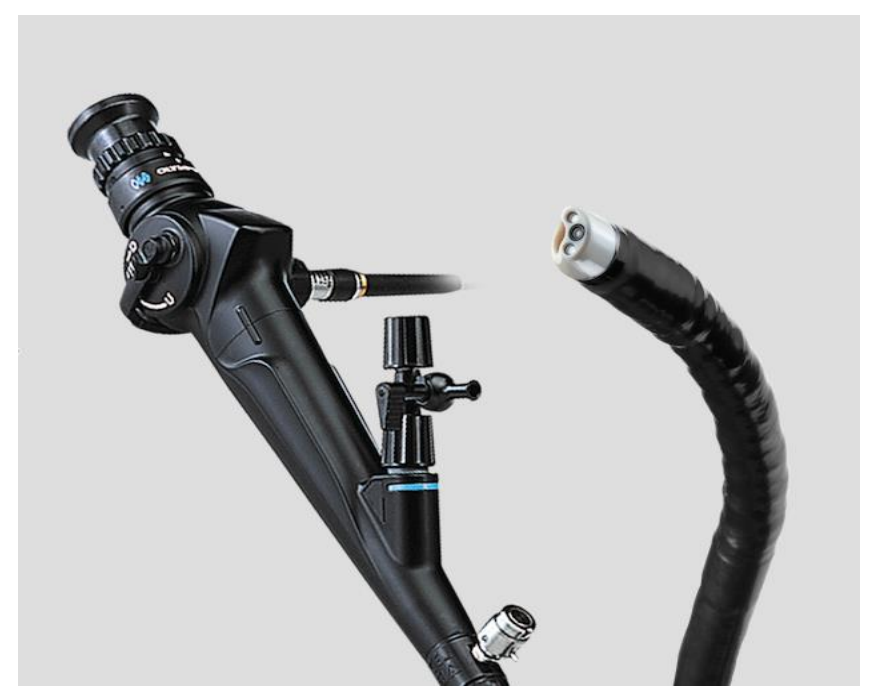

Abbildung 6: Fiberoskop zur flexiblen Zystoskopie: Typ CYF-VH bereitgestellt von Olympus Deutschland GmbH

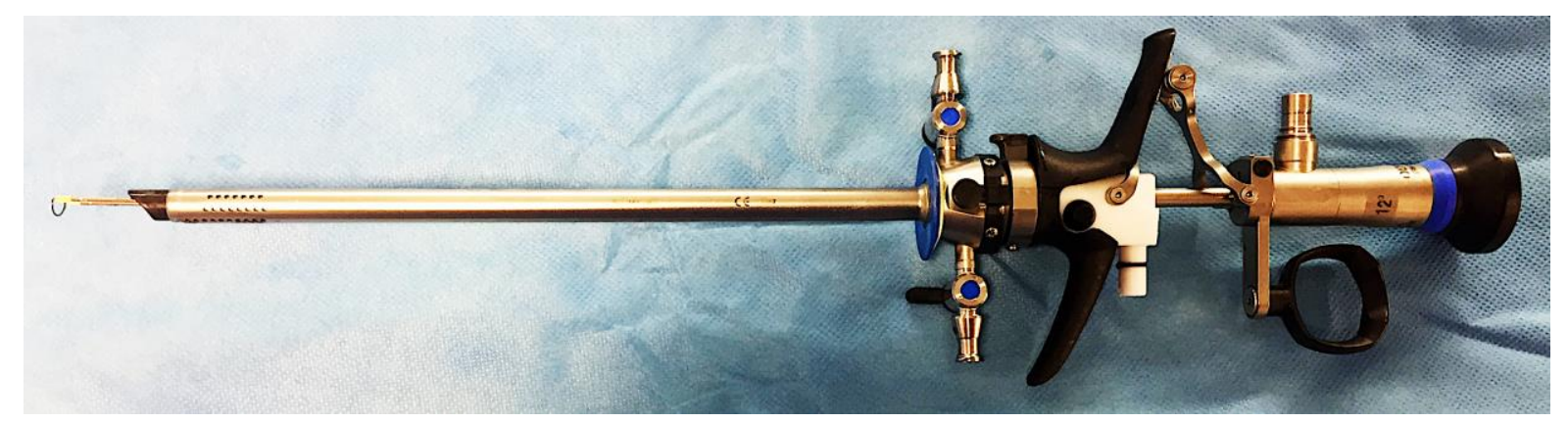

Abbildung 7: Starres Resektoskop mit beweglicher Resektionsschlinge Firma Olympus (eigene Bildersammlung)

\subsubsection{Weißlichtzystoskopie}

Spezielle Zusatzgeräte oder Spülungen sind bei der Weißlichtzystoskopie nicht erforderlich. Diese entspricht seit über 100 Jahren dem Goldstandard und wird sowohl im ambulanten Bereich als auch unter stationären Bedingungen durchgeführt. Die zystoskopische Detektionsrate von Blasenkarzinomen liegt bei knapp $80 \%$ und ist erfahrungsabhängig (Bichler et al. 1998b). Probleme in der Diagnostik bereiten Tumoren in anatomisch schlecht einsehbaren Arealen der Harnblase,

z. B. Divertikel und Tumoren mit einer flachen, strukturell dem normalen Urothel ähnlichen Textur, z. B. das Carcinoma in situ (Millan-Rodriguez et al. 2000; Rink et al. 2013). Zusätzlich beschreibt die Histologie noch zu oft residuales Tumorgewebe, sodass eine Nachresektion 
angestrebt werden muss. Ursächlich ist vermutlich die schlechte Beurteilbarkeit der Tumorausläufer am Resektionsrand (Brauers et al. 2001; Schwaibold et al. 2006).

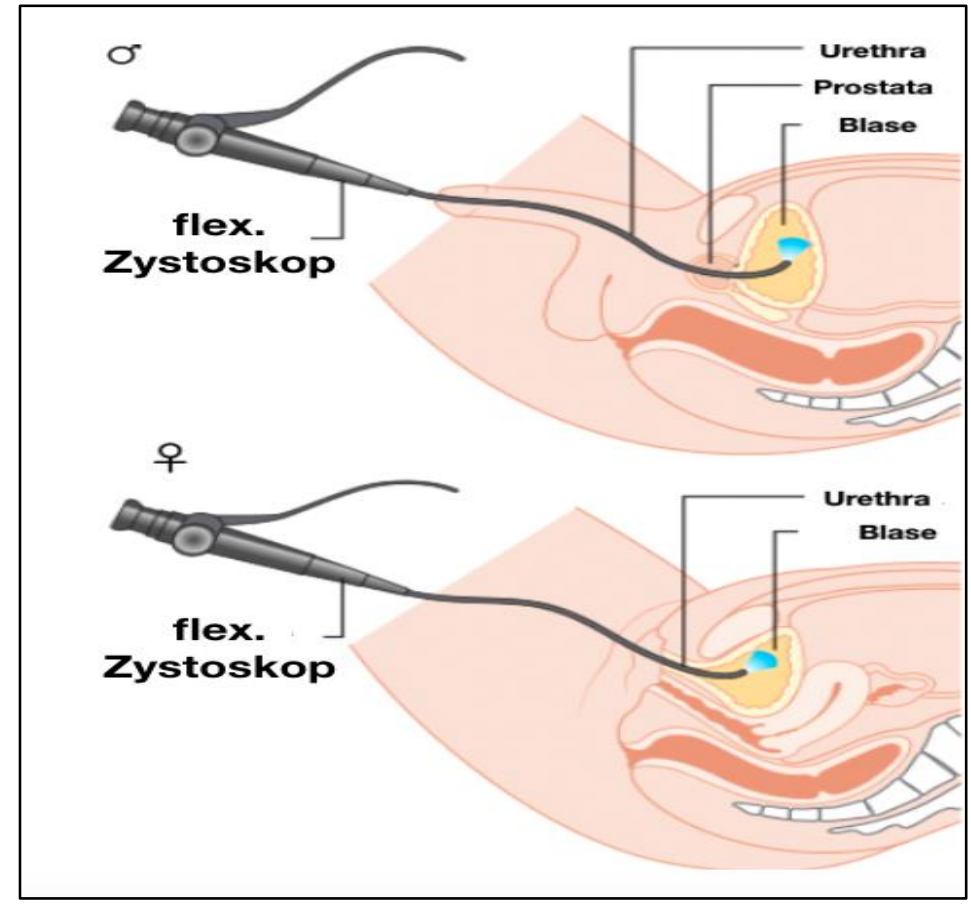

Abbildung 8: Schematische Darstellung einer Zystoskopie

(oben: beim Mann; unten: bei der Frau) (bereitgestellt von Cancer Research UK /Wikimedia Commons)

\subsubsection{Blaulichtzystoskopie; Photodynamische Diagnostik (PDD)}

Eine Fluoreszenzmarkierung urothelialer Tumoren wurde bereits 1965 erfolgreich mit Tetracyclinen durchgeführt (Whitmore und Bush 1965). Kriegmair et al. beschrieben 1994 erstmals die Anfärbung mit 5-ALA, einem Produkt der Hämbiosynthese. Problem war die schlechtere Aufnahme in die Zelle über die geringe Lipophilie. Eine Verbesserung war der Hexylester Hexylaminolävulinat (Hexvix ${ }^{\circledR}$ ) (Burger et al. 2009). Aufgrund der höheren Lipophilie kam es zu einer besseren Aufnahme in die Zelle über Diffusion. Da Tumorzellwände durchlässiger sind als Zellwände normaler Urothelien (vermutlich über eine Up-Regulation von transmembranösen Transporterproteinen) und die Aktivität der Ferrochelatase dort vermindert ist, reichert sich Hexvix ${ }^{\circledR}$ vermehrt an (Nakai et al. 2016). Circa eine bis drei Stunden präoperativ wird Hexaminolävulinat in die Blase instilliert. Die Moleküle werden über eine Bestrahlung mit Licht der Wellenlängen 380-450 nm über eine Absorption zum Fluoreszieren gebracht (Aussenden von einem schmalen Lichtband um $635 \mathrm{~nm}$, Abbildung 9) und über ein passendes Blaulichtzystoskop sichtbar gemacht (Bichler et al. 1998b). Hierdurch gelingt es selbst flache Läsionen wie Cis zu detektieren. Die Rate an zusätzlich detektierten Blasenkarzinomen liegt nach 
Studienlage bei knapp über 20 Prozent (Burger et al. 2013; Yuan et al. 2013). Seit Jahren wird PDD von der EAU zur Blasenkarzinomdiagnose empfohlen, obwohl eine anerkannte Schwäche die relativ geringe Spezifität ist (Fritsch und Ruzicka 2006). Die zusätzliche Detektionsrate von Cis liegt bei ca. 40 \% (O'Brien et al. 2013; Yang 2014). Hieraus ergibt sich paradoxerweise keine Verbesserung der Progressionsrate oder des Langzeitüberlebens (Rink et al. 2013; Yang 2014; Yuan et al. 2013).

Besonders bei Nachresektionen oder nach Instillationstherapien mit BCG oder Mitomycin ist die Spezifität stark vermindert (Stenzl et al. 2010). Hieraus ergibt sich die Zulassungsbeschränkung von Hexvix ${ }^{\circledR}$ auf mindestens 90 Tage nach vorheriger BCGBehandlung. Neben einer zusätzlichen Invasivität durch die präoperative Instillation von Hexaminolävulinat spielen insbesondere die Zusatzkosten durch eine spezielle Lichtquelle, ein spezielles Lichtleitkabel sowie Zusatzoptiken eine Rolle. Die Kosten von circa $500 €$ für Hexaminolävulinat werden durch eine Zusatzcodierung erstattet. Für den ambulanten Bereich wurde bereits eine flexible Variante vonseiten der Industrie entwickelt. Die Detektionsrate scheint in kleineren Fallserien aber nicht an die rigide PDD-Diagnostik heranzukommen (Witjes et al. 2005).

Aus diesen Gründen sowie der zusätzlichen Invasivität wird das PDD-Verfahren trotz der Empfehlung sowohl der Europäischen als auch der Deutschen Gesellschaft für Urologie fast ausschließlich im stationären Bereich eingesetzt. 


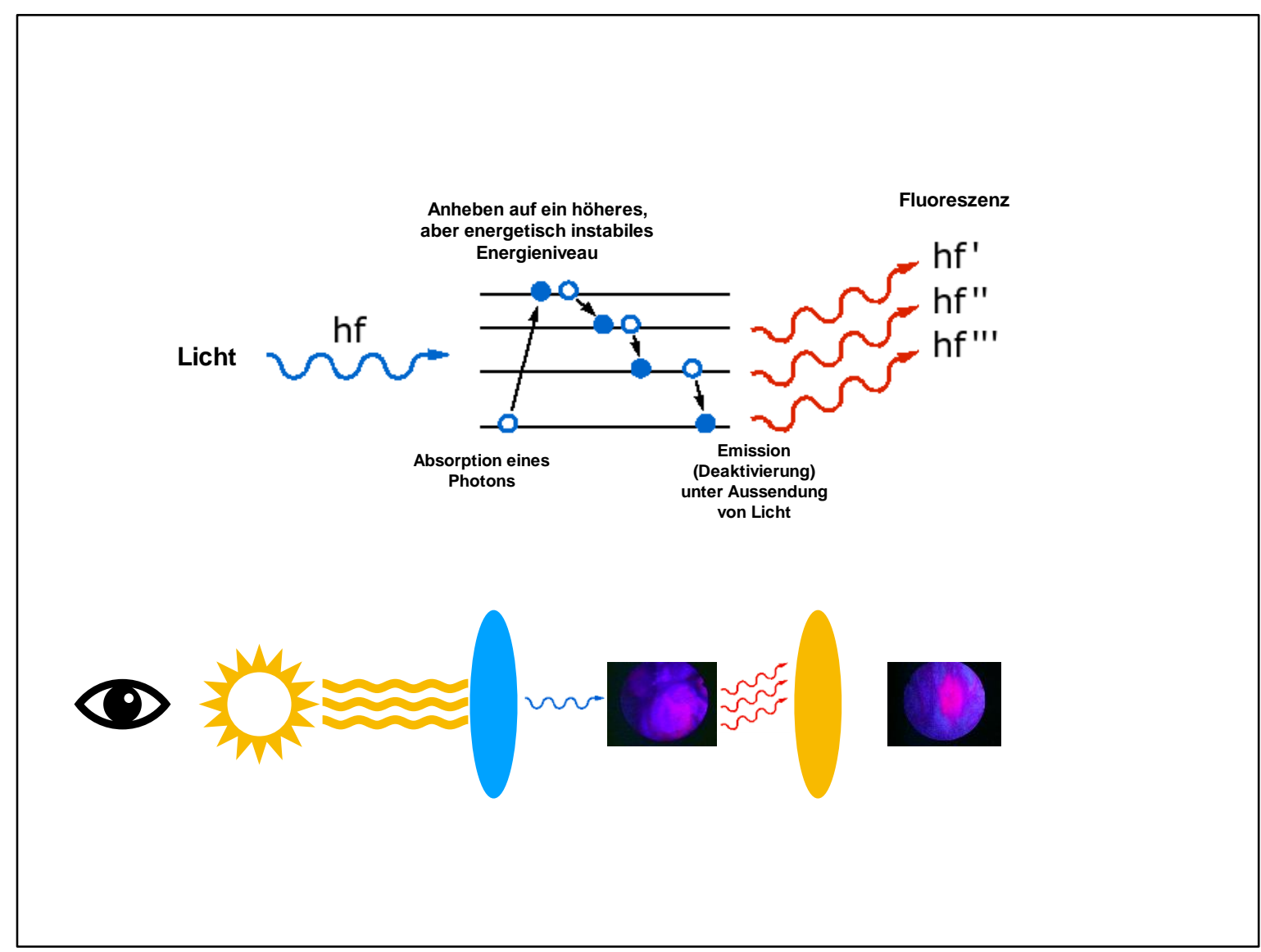

Abbildung 9: Prinzip der Fluoreszenzzystoskopie

Im Fluoreszenzmodus gibt es 2 Filter. Der erste filtert blaues Licht aus einem weißen Spektrum, dieses induziert die Fluoreszenz im proliferierenden Gewebe. Über den zweiten Filter wird die rote Fluoreszenz dann sichtbar gemacht. (eigene Bildersammlung) 


\subsubsection{NBI-Zystoskopie}

Die ersten Ideen zur NBI-Bildgebung entstanden um 1999. Mit einem NBI-Endoskop-Prototyp, entwickelt im National Cancer Center Hospital East, Japan, unter dem Einfluss von Dr. Sano, wurden ab Dezember 1999 erste Studien im Labor durchgeführt (Stein et al. 2001). Über eingeführte optische Fasern wurden gleichzeitig Qualität und Quantität des reflektierten Lichtes aufgezeichnet und ausgewertet. Die Forschungsgruppe fand, dass die definierte Wellenlänge um $415 \mathrm{~nm}$ eine Kontrasterhöhung zwischen Gefäßen und Schleimhaut bewirkt. Während die Erstgenerationsgeräte Probleme in der Helligkeit offenbarten, erlangte die NBI-Endoskopie ab der zweiten Generation vor allem in der Gastroskopie und später in der Koloskopie Aufmerksamkeit. Es konnte in einigen unkontrollierten Studien eine Überlegenheit insbesondere bei flachen Läsionen und intraepithelialen Neoplasien gezeigt werden (Chiu et al. 2007; Dekker et al. 2007; Su et al. 2006). In der Urologie hielt die NBI-Endoskopie erst ab circa 2008 Einzug. Es gibt bereits Studien, die eine Überlegenheit hinsichtlich der Detektionsrate ( $\mathrm{Li}$ et al. 2013; Zheng et al. 2012) gegenüber der Weißlichtzystoskopie zeigen. Naselli et al. (2012) konnten in einer prospektiv angelegten Studie sogar eine signifikant erhöhte Rezidivfreiheit nach einem Jahr bei Verwendung der NBI-Technik nachweisen. Insgesamt scheint eine genaue Beurteilung der Technik und somit eine Übernahme in die Leitlinien aufgrund der schmalen Datenlage und fehlender Vergleiche mit PDD noch zu früh.

\subsubsection{Physikalische Grundlagen}

Die physikalischen Grundlagen der NBI-Technik gehen zurück auf die Lehren der Optik. Licht wird, wenn es auf einen Körper trifft, teilweise von der Oberfläche zum Auge des Betrachters reflektiert und dort über die Retina in eine Farbinformation umgewandelt. Partiell gelangt es in den Körper und wird dort entweder absorbiert oder gestreut. Absorbiertes Licht wird dann in Wärme umgewandelt. 


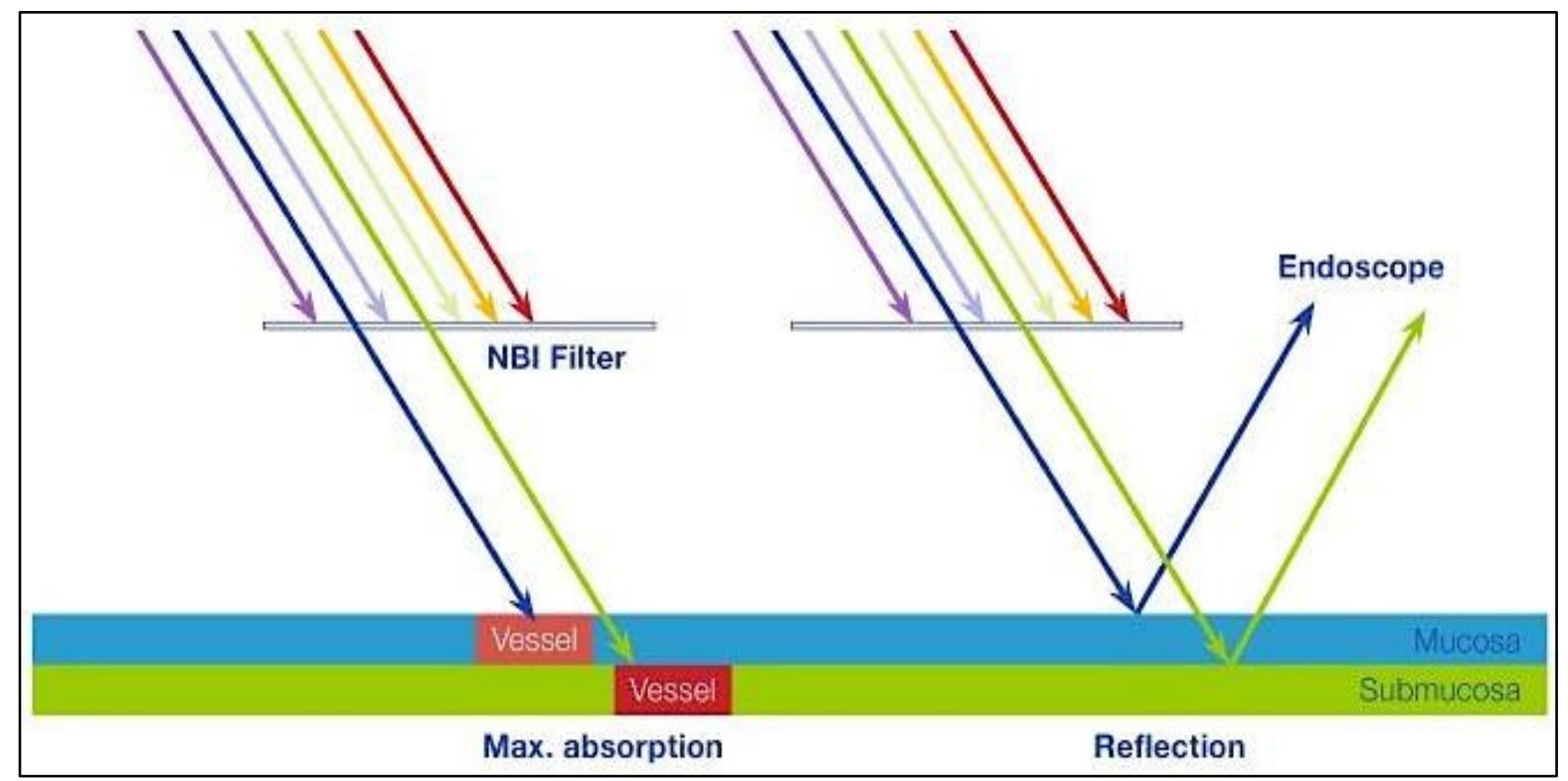

Abbildung 10: Darstellung des „NBI-Prinzips“

Im NBI-Modus wird durch das Vorlegen einer Blende der blaue (um $415 \mathrm{~nm}$ ) und grüne (um $540 \mathrm{~nm}$ ) Wellenlängenanteil herausgefiltert. (mit freundlicher Genehmigung von Olympus Deutschland GmbH)

NBI-Endoskope bedienen sich über einen rotierenden Filter definierter Spektren des Lichtes. Sichtbares Licht zwischen circa $380 \mathrm{~nm}$ und $780 \mathrm{~nm}$ Wellenlänge wird auf blaues Licht um 415 $\mathrm{nm}$ und grünes Licht um $540 \mathrm{~nm}$ reduziert (Abbildung 10). Das kurzwellige blaue Licht penetriert dabei nur bis in die oberflächliche Mucosa, die Schleimhautkapillaren werden hier in hohem Kontrast dargestellt. Das grüne Licht hat aufgrund der längeren Wellenlänge auch eine größere Gewebeeindringtiefe und kann somit tiefer liegende Gefäße in der Submucosa sichtbar machen. Beide Lichtspektren sind Absorptionsmaxima des Hämoglobins. Somit gelingt es, dass superfizielle Kapillaren über das kurzwellige blaue Licht als braun erscheinen, während das langwellige grüne Licht Gefäße, vor allem Tumorvenen, tief in der Mucosa und Submucosa in etwas bläulichem Farbton wiedergibt. Somit sollen oberflächliche, stark vaskularisierte, aber auch tiefer gelegene infiltrativ wachsende Tumoren identifiziert und aufgrund der unterschiedlichen Optik bestenfalls einer bestimmten Tiefeninvasion zugeordnet werden. 

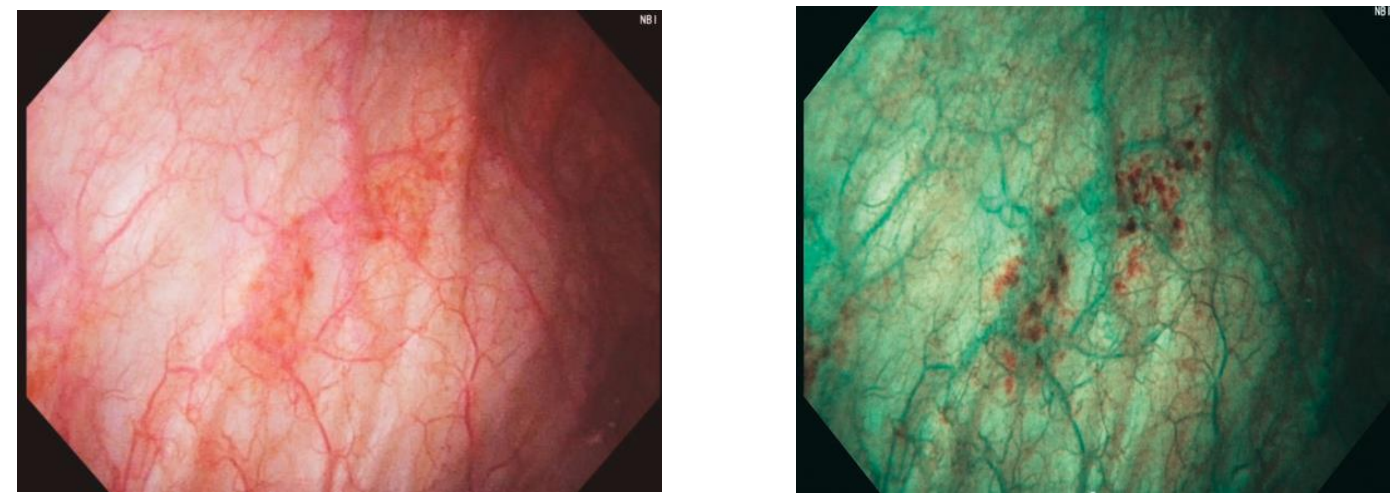

Abbildung 11: Vergleich Blasenschleimhaut in Weißlicht mit NBI-Technik.

Die malignomsuspekte Konfiguration wird lediglich in NBI deutlich sichtbar. Histologisch bestätigt sich ein Carcinoma in situ. (mit freundlicher Genehmigung durch Olympus Deutschland GmbH)

Die Kosten für die NBI-Technik beschränken sich auf eine zusätzliche Lichtquelle und NBIfähige Optik. Eine zusätzliche Invasivität ergibt sich nicht.

Die möglichen Vorteile dieser Technik sind derzeit Gegenstand verschiedener Untersuchungen. Ein Einsatz in der Primärresektion ist sinnvoll ((Herr 2015; Naselli et al. 2012). Es werden folgende Verbesserungen gegenüber der Weißlichttechnik postuliert:

- Erhöhte Sensitivität bei Tumoren (94,3 \% vs. 84,8 \% (Zheng et al. 2012)

- Reduktion der Rezidivhäufigkeit (51,4 \% vs. 32,9\% (Naselli et al. 2012)

- Verlängerung des rezidivfreien Überlebens um 16 Monate (Herr und Donat 2011)

- Bessere Erkennung flacher Neoplasien (Li et al. 2013)

\subsubsection{Qualitative Beeinträchtigung durch Hexaminolävulinat}

Bereits im Vorfeld dieser Arbeit erfolgte eine präliminäre Untersuchung [ $n=14$ Männer $+n=5$ Frauen] um eine, wenn auch nur theoretisch denkbare Veränderung des Bildes der NBIZystoskopie durch Hexvix ${ }^{\circledR}$ auszuschließen. Bei hochgradigem Verdacht auf ein Blasenkarzinom erfolgte die diagnostisch-endoskopische Untersuchung in Weißlicht und NBI. Nach makroskopischer Bestätigung der Diagnose wurde die Zystoskopie im Rahmen der Resektion nach Hexaminolävulinat-Instillation in NBI und PDD durchgeführt. Im Vergleich der beiden NBI-Bilder (diagnostische und therapeutische Endoskopie) ergaben sich keine Unterschiede. Damit ist eine Veränderung des NBI-Bildes durch Hexvix ${ }^{\circledR}$ nahezu ausgeschlossen [Datensammlung Prof. Wechsel, unveröffentlicht]. Es bestehen jedoch Hinweise für eine erniedrigte Spezifität nach vorheriger Instillationstherapie (Cauberg et al. 2010). 


\subsubsection{NICE-Kriterien von Iwatate bei kolorektalen Tumoren}

Die NICE-Kriterien (NBI International Colorectal Endoscopic Classification) von Iwatate gehen zurück auf die Zeiten der ausschließlich gastroenterologischen Verwendung von NBI. NarrowBand Imaging wurde 1999 in Japan entwickelt und hat die Chromoendoskopie (Anfärben der Schleimhaut mit Indigo-Carmin) aufgrund ihrer einfachen Verwendung und leichten Verfügbarkeit teilweise abgelöst.

Iwatate klassifizierte kolorektale Polypen in der Gastroenterologie in drei Typen und unterschied somit nicht nur zwischen benignen, adenomatösen und kanzerösen Läsionen, sondern gab gleichzeitig eine detailgenaue Angabe zur Notwendigkeit einer Resektion (resect and discard-Strategie) (Iwatate et al. 2012) und zur Tiefe der Invasion (Hattori et al. 2014). Hattori und Iwatate gaben mit ihren Untersuchungen den Anstoß zu einer international anerkannten Klassifikation kolorektaler Polypen. Diese werden in drei Grade hinsichtlich ihrer Farbe, Gefäße und Oberfläche unterschieden. Anhand dessen wird zwischen hyperplastischen Läsionen, benignen Adenomen oder bösartigen Karzinomen differenziert und intraoperativ ein Procedere festgelegt (s.u. Tabelle 1 und Abbildung 12) Die Differenzierungsqualität zwischen den einzelnen Typen lag jeweils bei über 90 \% (Utsumi et al. 2015), wobei bei Gruppe III noch zwischen IIIa und IIIb unterschieden wurde.

Die neu auf den Markt gebrachten, hochauflösenden Geräte haben die Detektionsrate und vor allem die Konfidenzangabe (bigh confidence vs. low confidence) in der Gastroenterologie nochmals verbessert (Iwatate et al. 2015; Utsumi et al. 2015). Ein kapilläres Muster analog den NICEKriterien für die Blase zu finden steht noch aus. Anspruch sollte es sein, eine ähnlich gute und anwendbare Differenzierung der Tumorstadien auf das Blasenkarzinom zu übertragen. 


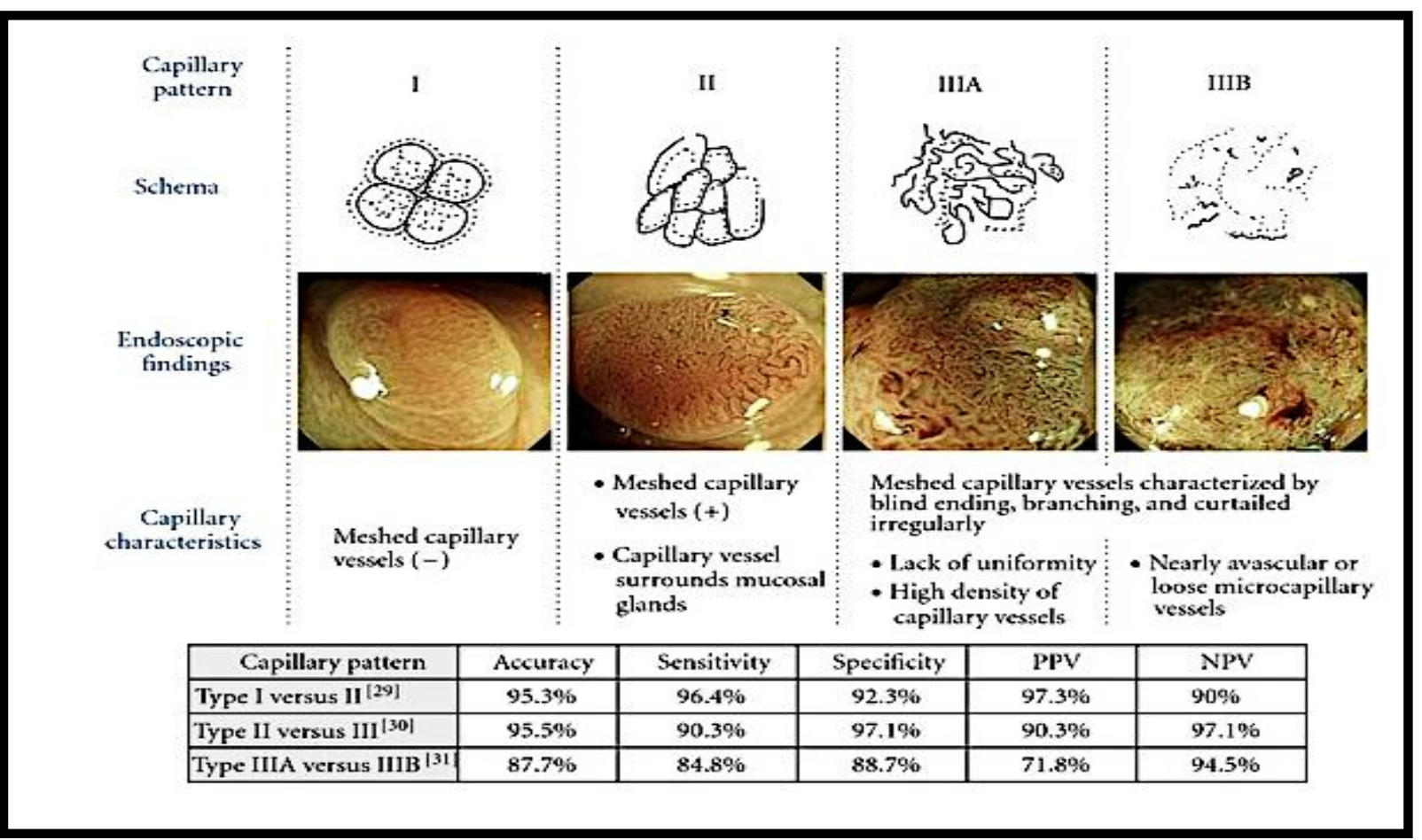

Abbildung 12: NICE-Klassifikation in der Gastroenterologie

Schematische Zeichnung mit dazugehörigen endoskopischen Befunden sowie den entsprechenden Gefäßmustern. Diagnostische Genauigkeit zwischen den einzelnen Gruppen der Klassifikation aus (Iwatate et al. 2012) mit Creative Commons-Lizenz.

Tabelle 1: NICE-Klassifikation nach Iwatate

\begin{tabular}{|c|c|c|c|}
\hline \multicolumn{4}{|c|}{ NICE classification. } \\
\hline & Type 1 & Type 2 & Type 3 \\
\hline Color & Same or lighter than background & $\begin{array}{l}\text { Browner relative to background (verify } \\
\text { color arises from vessels) }\end{array}$ & $\begin{array}{l}\text { Brown to dark brown relative to } \\
\text { background; sometimes patchy whiter } \\
\text { areas }\end{array}$ \\
\hline Vessels & $\begin{array}{l}\text { None, or isolated lacy vessels may be } \\
\text { present coursing across the lesion }\end{array}$ & $\begin{array}{l}\text { Brown vessels surrounding white } \\
\text { structures** }\end{array}$ & Has area(s) of disrupted or missing vessels \\
\hline $\begin{array}{l}\text { Surface } \\
\text { pattern }\end{array}$ & $\begin{array}{l}\text { Dark or white spots of uniform size, or } \\
\text { homogeneous absence of pattern }\end{array}$ & $\begin{array}{l}\text { Oval, tubular or branched white } \\
\text { structures** surrounded by brown yessels }\end{array}$ & Amorphous or absent surface pattern \\
\hline $\begin{array}{l}\text { Most likely. } \\
\text { pathology }\end{array}$ & Hyperplastic & Adenoma*** & Deep submucosalinvasive cancer \\
\hline Treatment & Follownp & Polypectomy/EMR/ESD & Surgery \\
\hline \multicolumn{4}{|c|}{$\begin{array}{l}\text { *Can be applied using colonoscopes with/without optical (zoom) magnification. } \\
\text { ** These structures (regular or irregular) may represent the pits and the epithelium of the crypt opening. } \\
\text { ***Type } 2 \text { consists of Vienna classification types } 3,4 \text { and superficial } 5 \text { (all adenomas with either low or high grade dysplasia, or with superficial } \\
\text { submucosal carcinoma). The presence of high grade dysplasia or superficial submucosal carcinoma may be suggested by an irregular vessel or } \\
\text { surface pattern, and is often associated with atypical morphology (e.g., depressed area). }\end{array}$} \\
\hline
\end{tabular}




\subsection{Primärtherapie des Blasenkarzinoms - die TUR-Blase}

Im therapeutischen Zentrum des Blasenkarzinoms steht die TUR-Blase. Verwendet wird für diese endoskopische OP-Technik ein Resektoskop, welches über die Harnröhre in die Blase vorgeschoben wird. Vergleichbar der Zystoskopie erfolgt die Tumorlokalisation mittels einer zumeist leicht abgewinkelten Optik $\left(0^{\circ} ; 12^{\circ} ; 30^{\circ} ; 70^{\circ}\right)$ unter gleichzeitiger Spülung der Blase mit einer elektrolytfreien Lösung bei geplanter monopolarer Resektion oder mit einer Kochsalzlösung bei geplanter bipolarer Resektion (TURis=transurethrale Resektion in saline). Bei der monopolaren Resektion wird am Patienten eine Elektrode (Erdung) angebracht, die zweite Elektrode ist die Resektionsschlinge. Bei der bipolaren Resektion ist die Leitelektrode und die Neutralelektrode jeweils in der Schlinge. Die Verwendung eines Resektoskopes anstelle eines Zystoskopes und die gleichzeitig bestehende Resektionsbereitschaft kann die sonst im Vorfeld durchgeführte Zystoskopie ersetzen, wenn die Diagnose eines Blasenkarzinoms oder eines resektionswürdigen Befundes bereits anderweitig gestellt wurde.

An dem Resektoskop befindet sich eine elektrische Schlinge, die mittels eines Schlittens am Außenschaft des Gerätes vor- und zurückbewegt werden kann. Unter Strom schneidet diese Schlinge das Gewebe und führt gleichzeitig eine Koagulation durch. Die Gewebestücke werden anschließend über den Außenschaft nach Entfernung des Innenanteils des Resektionsgerätes aus der Blase ausgespült.

Die Technik der Tumorresektion wirft mehrere Probleme auf. Zum einen werden technisch bedingt die Tumoren in aller Regel quasi in Portionen geschnitten und entfernt, zum anderen sind die Resektionsgrenzen mangels sicher definierter Tumorgrenzen nicht immer sicher einzuhalten. Insbesondere die Frage nach der Resektionstiefe (in das Blasengewebe hinein) ist schwierig bei bestehendem Risiko einer Blasenperforation.

Ein mögliches Vorgehen gegen diese Limitationen ist die en-bloc-Resektion (Herrmann et al. 2017; Struck et al. 2017). Hierbei wird der Tumor zumeist mit einer Kochsalzlösung submukös unterspritzt. Hierdurch kommt es zu einer Abhebung vom Untergrund. Anschließend wird der Tumor umschnitten und allschichtig (en bloc) entfernt. Das Argument, dass die Tumorlokalisation an der Vorderwand eine Einschränkung darstellt, konnte größtenteils entkräftet werden (Abotaleb et al. 2017). Das Risiko einer Perforation wird durch die Stärkenerhöhung der Blasenwand minimiert. Die Rezidivrate soll durch die Minimierung der Tumorimplantation und Verhinderung einer inkompletten Resektion vermindert werden (Sureka et al. 2014). Bisher liegen vielversprechende Daten nur aus Metaanalysen vor (Wu et al. 2016). 
Am häufigsten wird die sogenannte differenzierende Resektion durchgeführt. Nach Abtragen des sichtbaren papillären, soliden oder breitbasigen Tumoranteils wird an den Resektionsrändern auf $3 \mathrm{Uhr}, 6 \mathrm{Uhr}, 9 \mathrm{Uhr}$ und $12 \mathrm{Uhr}$ eine weitere Probe entnommen. Zusätzlich wird am Tumorgrund tief reseziert (s. Abbildung 15; S. 30).

Bei nicht sicher freiem Tumorgrund oder bei höher aggressiven Tumoren erfolgt dann eine Nachresektion nach ca. 4-6 Wochen entweder zur Bestätigung der Tumorfreiheit oder zur Entfernung von Resttumorgewebe.

In Abhängigkeit von der histologischen Befundung war die Resektion kurativ, diagnostisch mit nachfolgenden Maßnahmen oder palliativ bei fortgeschrittenen und/oder inoperablen Tumoren zu bewerten (Abbildung 13).

Aus den Problemen der Resektion ergeben sich vor allem Verbesserungspotentiale in der Festlegung der Genauigkeit der Tumorgrenzen.

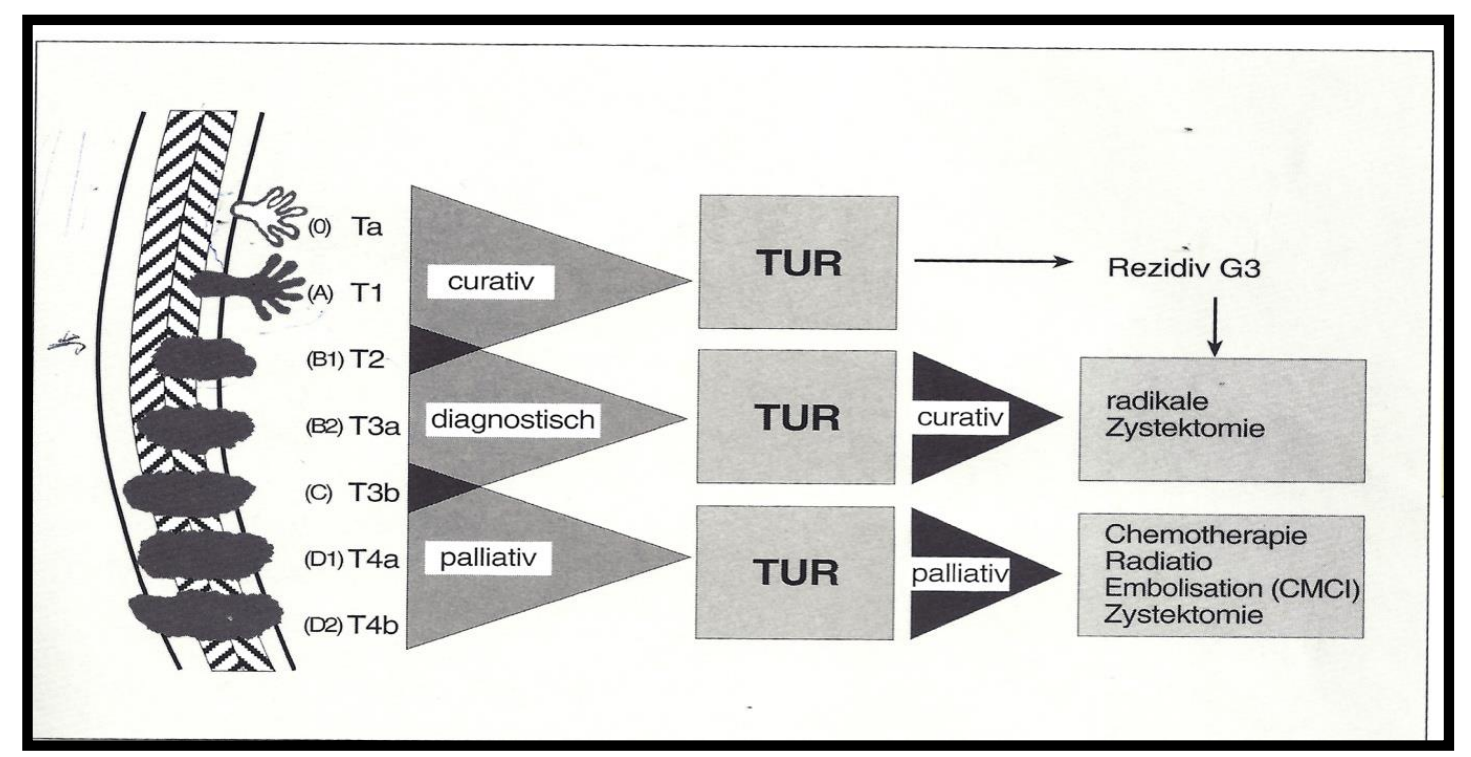

Abbildung 13: Die drei verfolgten Ziele einer TUR der Blase

Während niedrig maligne Tumoren so diagnostiziert und simultan kuriert werden können, dient die TUR-B bei fortgeschrittenen Stadien zusammen mit anderen Maßnahmen einer Palliation. (mit freundlicher Genehmigung von Bichler et al. 1998.) 


\subsection{Prognose}

Die Prognose des Blasenkarzinoms ist abhängig vom Stadium, Grading und der Therapie. Einen Aufschluss gibt Tabelle 2.

Tabelle 2: Überlebensdaten der jeweiligen Tumorstadien (adaptiert aus Bichler et al. (1998b)

\begin{tabular}{|c|c|c|c|}
\hline Jahre & Stadium & Therapie & Überleben \\
\hline 5 & pTaG1-pTaG2; N0, M0 & TUR-Blase (+PDD/NBI) & $96 \%$ \\
\hline 5 & pT1G3; N0; M0 & TUR-Blase & $64 \%$ \\
\hline 5 & pT1G3; N0; M0 & Zystektomie & $80 \%$ \\
\hline 5 & pT1G3; N0; M0 & Radiochemotherapie & $80 \%$ \\
\hline 5 & pT2; NX; MX & TUR-Blase & $45 \%-70 \%$ \\
\hline 5 & pT2; NO; MO & Zystektomie & $65 \%-75 \%$ \\
\hline 5 & pT3; NX; MX & Zystektomie & $20 \%-35 \%$ \\
\hline 5 & pT3; NX; MX & Radiochemotherapie & $30 \%$ \\
\hline 5 & pT4; NX; MX & Zystektomie & $6-25 \%$ \\
\hline 3 & pT4; NX; MX & $\mathrm{CMCl}^{*}$ & $15 \%$ \\
\hline 3 & M1 & MVAC** & $20 \%-25 \%$ \\
\hline 3 & M1 & Gemcitabine/Cisplatin & $35 \%$ \\
\hline
\end{tabular}




\section{Wissenschaftliche Fragestellungen}

In dieser präliminären Untersuchung sollen aufgrund des in der Einleitung beschriebenen Problemhintergrunds folgende Fragen hinsichtlich ihrer Möglichkeit zur endgültigen Beantwortung in größeren, multizentrischen Studien untersucht werden.

- Gibt es Kriterien in der Beurteilung des NBI-Bildes, die die folgenden Fragen beantworten?

- Können morphologische Aspekte des Tumors schematisiert werden?

- Gibt es morphologische Kriterien zur Bestimmung eines Cis?

- Können in der NBI-Technik nicht-maligne Tumoren sicher von malignen Tumoren unterschieden werden?

- Ist in der NBI-Technik eine Stadiendifferenzierung (Invasion/Grading) des Blasenkarzinoms möglich?

- Ist eine genaue Beurteilung des Tumorrandes, also des genauen Übergangs von pathologischem zu gesundem Gewebe möglich?

- Können alle tumorsuspekten Areale, die im Weißlicht oder der PDD-Technik detektiert werden, auch in der NBI-Technik gefunden werden?

- Ist die NBI-Technik in der Lage, zusätzliche Tumoren zur Weißlicht- oder der PDD-Technik qu detektieren?

Ziel der vorliegenden Pilotstudie ist eine grobe Evaluation der Wertigkeit und Praxisrelevanz des NBI-Verfahrens sowie die Erarbeitung in weiteren Studien zu prüfender Prämissen. Hieraus könnte sich mittelfristig eine kostengünstige, anwenderfreundliche und weniger belastende diagnostische Maßnahme vor allem für den niedergelassenen Bereich ergeben. 


\section{Methodik}

Die Studie wurde im Zeitraum vom Mai 2014 bis Dezember 2016 durchgeführt. Für die Untersuchung wurde ein positives Votum der Ethikkommission des Fachbereichs Medizin eingeholt. In die Untersuchung wurden Patienten als sogenanntes ,convenience sample inkludiert, bei denen eine Hämaturieabklärung mit hochgradigem Verdacht auf ein ursächlich vorliegendes Blasenkarzinom notwendig war oder die zu einer TUR-Blase durch einen niedergelassenen Kollegen nach ambulanter Detektion eines Blasentumors zugewiesen wurden.

Es wurden folgende Einschlusskriterien zugrunde gelegt:

- Z. n. Makrohämaturie mit Indikation zur Zystoskopie

- Z. n. Mikrohämaturie mit Indikation zur Zystoskopie

- Ambulanter Verdacht auf Blasenkarzinom mit Indikation zur TUR-Blase

- Multilokularität der Blasentumoren

Es wurden folgende Ausschlusskriterien zugrunde gelegt:

- Ablehnung zur Aufnahme in die Untersuchung

- Kontraindikation/fehlende Indikation für Hexvix®-Instillation

- Durchführung von $<$ drei bildgebenden Techniken

- Technische Fehlerquellen

- Intraoperativer Befund einer Tumorkompression von außen

- Nicht eingewiesener Operateur (Assistenzärzte in früher Ausbildung)

Alle im Rahmen der Studie untersuchten Patienten gaben im Zuge der operativen Aufklärung ihr Einverständnis zur Auswertung der intraoperativen Zystoskopiebefunde. Alle Operateure (mindestens Facharztstatus oder Studienleiter) wurden zur NBI-Technik durch theoretisches Material und Bildmaterial instruiert.

Bei zugewiesenen Patienten erfolgte die Zystoskopie nach entsprechender Aufklärung während der TUR-Blase in Weißlicht, PDD-Technik und NBI-Technik. Bei Patienten mit Verdacht auf ein Blasenkarzinom, welche entsprechend in der Klinik einer Primärdiagnostik unterzogen wurden, erfolgte die erforderliche Zystoskopie sowohl in Weißlicht als auch in NBI-Technik. 
Hier wurde die PDD-Technik erst im Rahmen der nachfolgenden TUR-Blase durchgeführt, um unnötige Instillationen des Photosensitizers zu vermeiden. Ein Ablaufalgorithmus zeigt Abbildung 14.

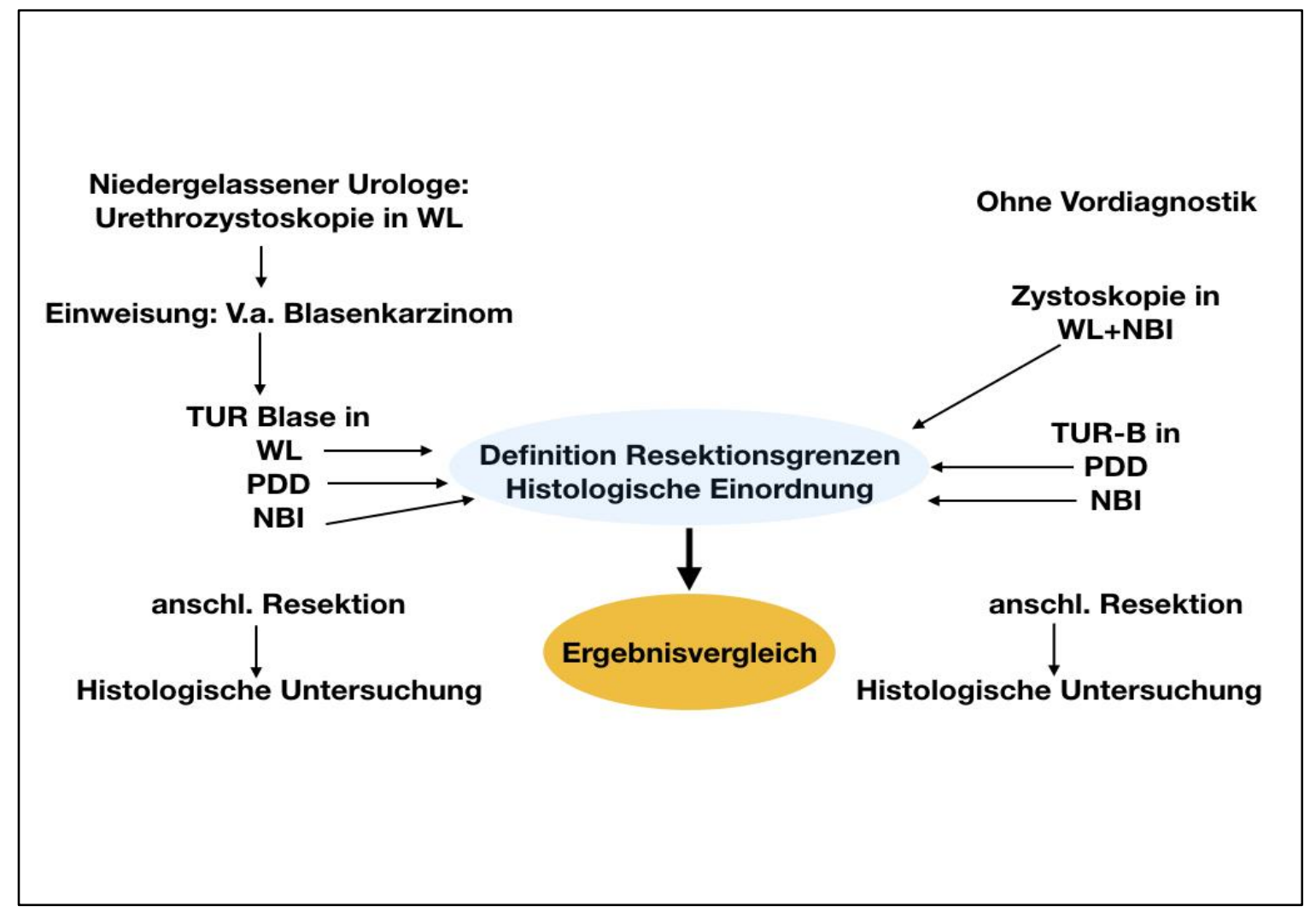

Abbildung 14: Ablaufschema der Blasenkarzinom-/Hämaturieabklärung

für Patienten mit ambulanter Vordiagnostik (links) und ohne Vordiagnostik (rechts) (eigene Bildersammlung)

Die Primärdiagnostik erfolgte mittels flexibler Zystoskopie (Weißlicht- und NBI-HD Zystoskop (flexibel) der Firma Olympus). Eine Übersicht der genauen Gerätebezeichnungen der verwendeten Instrumente zeigt folgende Tabelle:

Tabelle 3: Übersicht verwendeter optischer Geräte

\begin{tabular}{|c|c|c|}
\hline \multicolumn{2}{|r|}{ Funktion } & \multirow{2}{*}{$\begin{array}{l}\text { Gerätebezeichnung } \\
\text { WA03300A }\end{array}$} \\
\hline & Lichtleitkabel & \\
\hline \multirow[t]{6}{*}{ Weißlicht/NBI } & Zystoskop flexibel & CYF-5 \\
\hline & Videozystoskop HD flexibel & CYF VH \\
\hline & Starre Optik, $4 \mathrm{~mm}, 70^{\circ}$ & A22003A \\
\hline & Starre Optik, $4 \mathrm{~mm}, 12^{\circ}$ (Resektion) & A22001A \\
\hline & Kamerakopf & OTV-S7ProH-HD-L08 \\
\hline & Lichtleitkabel (PDD) & A93200A \\
\hline \multirow[t]{4}{*}{$P D D$} & Optik 4 mm, $70^{\circ}$ (PDD) & WA20018A \\
\hline & Optik, 4 mm, $12^{\circ}$ (PDD) (Resektion) & WA20016A \\
\hline & Kamerakopf & OTV-S7ProH-FD (PDD) \\
\hline & Videoadapter & AR-TF08E \\
\hline
\end{tabular}


Im Rahmen der Resektion wurden Randproben (s. Schema Abbildung 15) zum Nachweis residualen Tumorgewebes entnommen (differenzierende Resektion). Dabei wurde der Tumor makroskopisch mit einem ca. 2 bis $3 \mathrm{~mm}$ großen Sicherheitsareal reseziert. Kleine papilläre Tumoren wurden auf Verantwortung des Operateurs „mit einem Schlingenschlag“ sicher im Gesunden reseziert. Weiterhin wurden regelmäßig eine oder bei größeren Tumoren mehrere Proben aus dem Tumorgrund entnommen, ebenfalls mit der Frage nach residualer, ggf. muskulärer Infiltration. Es ergaben sich abhängig von der Histologie folgende residualen Tumorraten:

Tabelle 4: Statistische Übersicht zu den Residualtumorraten nach TUR-B nach Primärresektion, zweiter (Nachresektion) und dritter (Sicherheitsresektion) Resektion unterschieden nach Tumorstadien. Aus Bichler et al. (1998b)

\begin{tabular}{|cccccc|}
\hline $\begin{array}{c}\text { Primär- } \\
\text { resektion }\end{array}$ & $\begin{array}{c}\text { Nach- } \\
\text { resektion }\end{array}$ & $\begin{array}{r}\text { Sicherheits- } \\
\text { resektion }\end{array}$ \\
\hline TaG1 & 84 & 2 & $(2,4 \%)$ & 0 & \\
TaG2 & 57 & 7 & $(12,3 \%)$ & 2 & $(3,5 \%)$ \\
T1G1 & 11 & 6 & $(36,4 \%)$ & 2 & $(9,1 \%)$ \\
T1G2 & 29 & 5 & $(24,1 \%)$ & 3 & $(13,8 \%)$ \\
T2G2 & 15 & 10 & $(66,6 \%)$ & 5 & $(33,3 \%)$ \\
\hline & 196 & 30 & $(15,3 \%)$ & 12 & $(6,1 \%)$ \\
\hline
\end{tabular}

Im Rahmen dieser Untersuchung wurden die Randproben möglichst dicht am Resektionsrand genommen. Dieser wiederum wurde anhand des NBI-Bildes festgelegt. Eine negative Randprobe bedeutete eine erfolgreiche Tumorrandbeurteilung. 


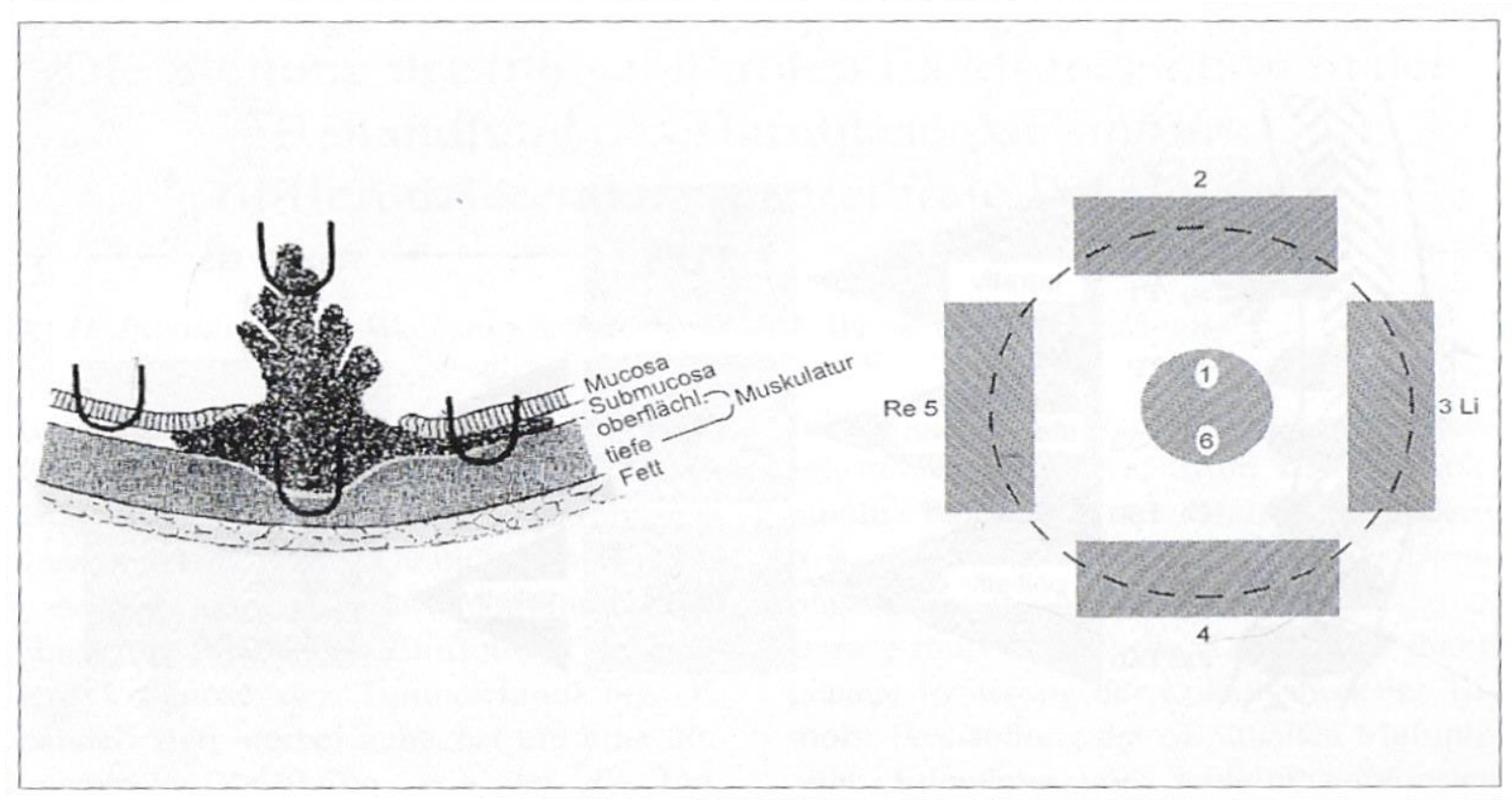

Abbildung 15: Schema zur Technik der differenzierenden TUR-Blase inklusive histologischer Veranschaulichung (links) aus Bichler et al. (1998b)

Die TUR-Blase wurde routinemäßig mit einem starren Resektoskop durchgeführt. Für das OPPflegepersonal und die jeweiligen Operateure wurde eine SOP inklusive schematischer Zeichnung entwickelt, anhand derer der Aufbau und die technischen Einstellungen präoperativ vorgenommen werden konnten .

Nach dem Sammeln von Bilddateien und Erfahrungen wurde versucht, die Histologiebefunde den intraoperativen Bildern zuzuordnen. Bei den ersten 40 Patienten wurde der Histologiebefund kognitiv mit den intraoperativen Bildern fusioniert. Gemeinsamkeiten und Unterschiede der Stadien wurden heraus- und schematisch aufgearbeitet (s. Tabelle 5) und Kriterien für die Detektion von Suspizien erfasst. Dabei orientierte sich die Kriterienerarbeitung an den Vorgaben der Gastroenterologie bei Darmtumoren.

Nach Abschluss dieser Lernphase wurden im weiteren Verlauf bei 24 Patienten (mit insgesamt 26 Tumorbefunden; multilokuläre Blasentumoren wurden getrennt beurteilt) Tumorstadium und Tumorkonfiguration vor Erhalt der Histologie prognostiziert und der Resektionsrand in NBITechnik festgelegt.

Die Tumorrandbeurteilung wurde über die Positivität oder Negativität (in Tabelle 7 als ,+‘ (erfolgreiche Tumorrandbeurteilung) oder ,-` (erfolglose Tumorrandbeurteilung)) der Randproben in einer NBI-gesteuerten TUR-Blase beurteilt. Waren alle Randproben tumorfrei, war die NBI gesteuerte TUR-Blase erfolgreich; war auch nur eine der Randproben positiv, wurde von einer ungenauen Tumorrandbeurteilung in NBI ausgegangen. Zudem wurden die 
unterschiedlichen Bildtechniken hinsichtlich Ihrer Genauigkeit bzgl. Tumordetektion und Tumorrandbeurteilung verglichen. Um von einer positiven Beurteilung in Weißlicht und PDD auszugehen, musste der Tumorrand lediglich klar vom normalen Urothel abzugrenzen sein. Da die endgültige Resektion in NBI durchgeführt wurde, liegt für die Tumorrandbeurteilung nur dieser optische Surrogatparameter und kein histologisches Ergebnis vor. Ähnlich verhält es sich bei der Tumordetektion. Ein klares Erkennen eines Tumors mit abschließender korrekter Dignitätseinschätzung wurde positiv $\left(,+^{`}=\right.$ richtig positiv/richtig negativ) bewertet. Eine falsch negative oder falsch positive Dignitätseinschätzung oder das „Übersehen“ eines Tumors wurde negativ (,- $)$ bewertet.

Eine feste Sequenz der Untersuchung wurde nicht festgelegt. Zumeist wurde mit PDD begonnen, da sich der Photosensitizer bei zunehmender OP-Dauer auswaschen kann.

Die Ergebnisse wurden deskriptiv und mittels einfacher prozentualer Bestimmungen verglichen. Fehlende Übereinstimmungen wurden einzeln analysiert. Aufgrund der geringen Datenmenge wurde auf eine Signifikanzbeurteilung verzichtet.

Von den 24 Patienten waren 13 männlich und 11 weiblich. Die Patienten waren zwischen 29 und 88 Jahre alt (Durchschnittsalter 71,5) Es wurde die WHO-Klassifikation von 1974 (s. 1.2.1 TNM-System) zugrunde gelegt.

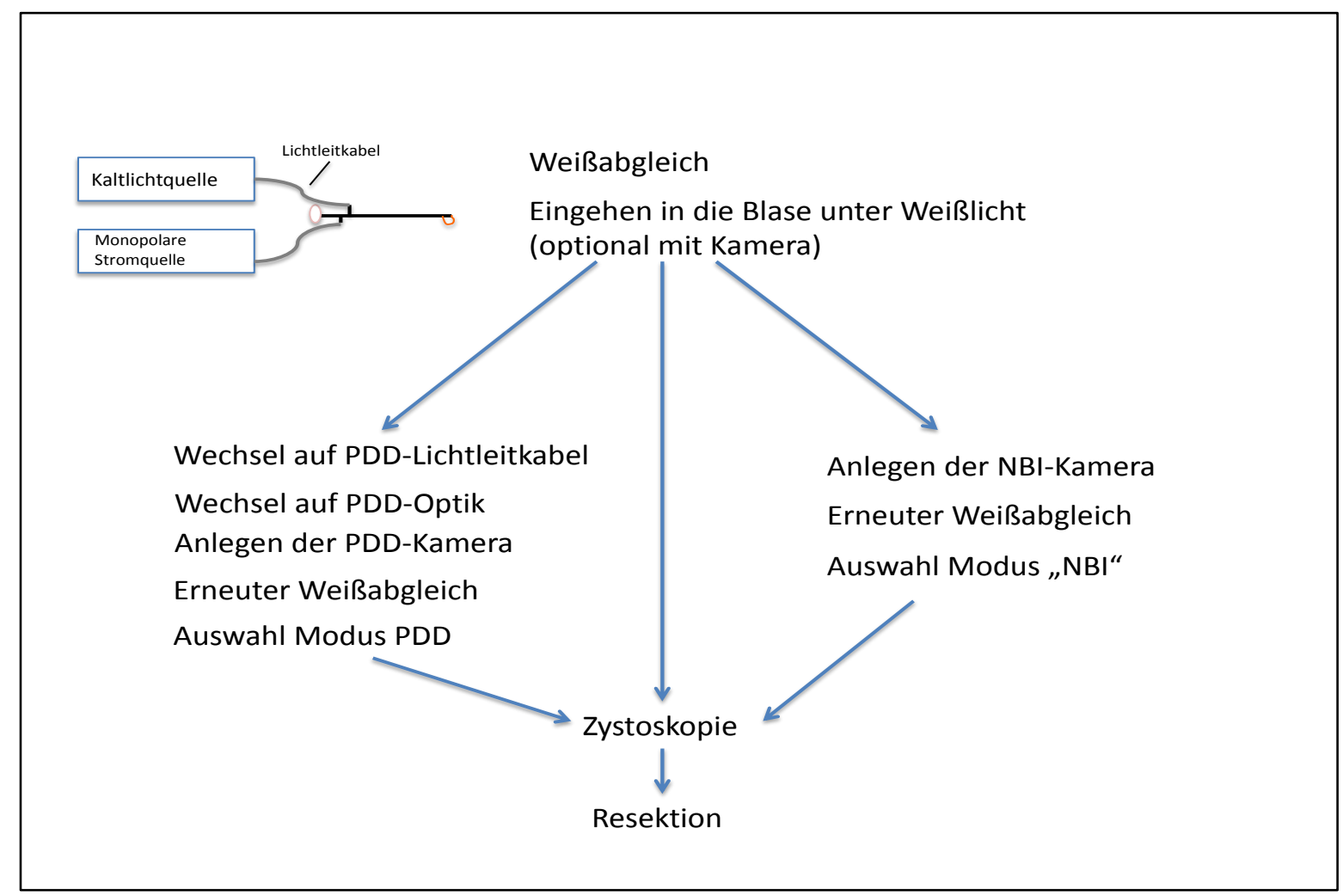

Abbildung 16: SOP zum Ablauf und Aufbau der TUR-Blase in den verschiedenen bildgebenden Techniken. 


\section{Ergebnisse}

\subsection{Allgemeines}

Im Zeitraum von Mai 2014 bis Dezember 2016 wurden insgesamt 648 TUR-Blasen durchgeführt. Nicht in die Auswertung wurden genommen:

- $\mathrm{n}=53$, da nur entweder eine Weißlichtzystoskopie mit PDD oder eine Weißlichtzystoskopie mit zusätzlicher NBI-Technik vorgenommen wurde. Die Kombination aller 3 Verfahren konnte bei je einem Geräteturm mit PDD oder NBI bei zeitgleichem Eingriff in 2 OP-Räumen nicht stattfinden.

- $\mathrm{n}=36$ mit technischen Fehlerquellen, z. B. Defekt des NBI- oder PDD-Gerätes.

- $\mathrm{n}=168$, da PDD nicht indiziert war

- $\mathrm{n}=230$, da diese TUR-Blase als Lehr-OP durch einen in Weiterbildung befindlichen Assistenten erfolgte

- $\mathrm{n}=32$, da nur unzureichende Befunderstellung

- $\mathrm{n}=65$, mit intraoperativer Feststellung, dass es sich entgegen der Einweisungsdiagnose nicht um ein Blasenkarzinom handelte, sondern ein von außen infiltrierender Tumor vorlag oder eine andere fehlerhafte Zuweisungsdiagnose

- $\mathrm{n}=40$ Patienten in der präliminären Untersuchung zur Erweiterung der NBI-Kriterien nach Iwatate

Letztlich konnten $\mathrm{n}=24$ Patienten mit insgesamt 26 Blasenkarzinomen (Multilokularität als Einschlusskriterium) in die Auswertung (Weißlicht, PDD und NBI) eingeschleust werden. Von den 24 Patienten waren $n=13$ männlich und $n=11$ weiblich. Die Patienten waren zwischen 29 und 88 Jahre alt (Durchschnittsalter 71,5). 


\subsection{NBI-Morphologie von Blasenkarzinomen}

\section{○ Können morphologische Aspekte des Tumors schematisiert werden?}

In einem ersten Schritt wurden bei 40 Patienten Kriterien der Morphologie für die NBI-Technik analog Iwatate erarbeitet. Dazu wurden die suspekten Areale in der NBI-Technik fotografiert und mit der histologischen Befundung verglichen. Unterschiede fanden sich in der Gefäßmorphologie. Diese sind in der folgenden Tabelle zusammengefasst, die Schemaskizze (

Abbildung 17) verdeutlicht den Befund. Es gelang, morphologische Aspekte des Tumors zu schematisieren.

Tabelle 5: Schema für die Bewertung von Blasentumoren in der NBI-Zystoskopie

\begin{tabular}{|l|l|l|l|}
\hline Tumormorphologie & $\begin{array}{l}\text { Urozystitis } \\
\text { Ummantelung } \\
\text { eines Gefäßes mit } \\
\text { glasiger, } \\
\text { ödematöser } \\
\text { Schleimhaut }\end{array}$ & $\begin{array}{l}\text { Läßig hoher } \\
\text { Gefäßanteil, ca. 1/3 des } \\
\text { exophytischen Anteils, } \\
\text { verzweigt, } \\
\text { „blumenkohlartig“ }\end{array}$ & $\begin{array}{l}\text { Kleiner, aber } \\
\text { unregelmäßiger } \\
\text { Gefäßanteil und } \\
\text { Gefäßverlauf } \\
\text { zuungunsten einer } \\
\text { soliden Formation } \\
\text { Geringer } \\
\text { Schleimhautanteil }\end{array}$ \\
\hline Umgebende Gefäße & Feinkapilllär & $\begin{array}{l}\text { Retikulär, feinkapilläre } \\
\text { bis mittelgroße Kaliber } \\
\text { (Kalibersprünge) }\end{array}$ & $\begin{array}{l}\text { Balkig, irregulär, tiefe } \\
\text { Gefäße (schwächer } \\
\text { kontrastreich) }\end{array}$ \\
\hline
\end{tabular}
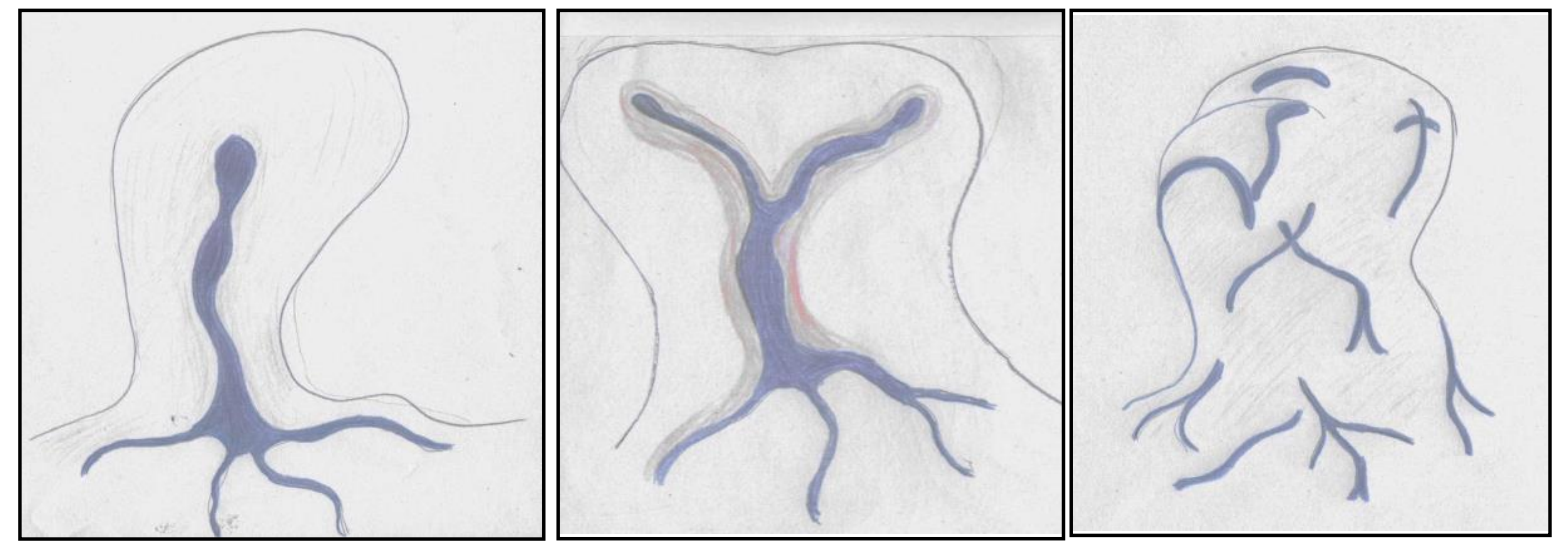

Abbildung 17: Schematische Zeichnungen der Morphologie einer Urozystitis, eines low grade-Tumors und eines bigh grade-Tumors. (von links nach rechts)

\section{$\circ$ Gibt es morphologische Kriterien zur Bestimmung eines Cis?}

Für ein Cis konnte aufgrund der geringen Häufung in der Studie keine Schematisierung erfolgen. 


\subsection{Kontrolle der eigenen Befunde}

Bei den folgenden $n=24$ Patienten wurde das durch den NBI-Befund prognostizierte Tumorstadium schriftlich festgelegt und später mit dem histologischen Befund verglichen.

Es ergab sich eine hohe Übereinstimmung zwischen NBI-basierter Prognose und dem tatsächlichen histologischen Ergebnis (s. Tabelle 6)

Tabelle 6: Ergebnisse hinsichtlich der NBI-Prognose und der Tumorrandbestimmung.

\begin{tabular}{|c|c|c|c|c|}
\hline Pat. Nr. & NBI-Prognose & $\begin{array}{l}\text { Histologischer } \\
\text { Befund }\end{array}$ & Randproben $*$ & Bemerkung \\
\hline 1 & Ta low grade & Ta low grade & tumorfrei & \\
\hline 2 & T2 high grade & T2 high grade & a) & \\
\hline 3 & Urozystitis & Urozystitis & b) & \\
\hline 4 & Urozystitis & Urozystitis & b) & \\
\hline 5 & $\begin{array}{l}\text { Tumor nicht urothelialen } \\
\text { Ursprungs, } \\
\text { Plattenepithelkarzinom? }\end{array}$ & $\begin{array}{l}\text { granulomatöse } \\
\text { Entzündung }\end{array}$ & b) & 1 \\
\hline 6 & Leukoplakie & Leukoplakie & b) & \\
\hline 7 & Urozystitis & Urozystitis & b) & \\
\hline 8 & Ta low grade & Ta low grade & tumorfrei & \\
\hline 9 & Urozystitis & Urozystitis & b) & \\
\hline 10 & Ta low grade & Ta low grade & $\begin{array}{l}\text { positiv in } \\
\text { Nachresektion }\end{array}$ & 2 \\
\hline 11 & Ta low grade & Ta low grade & tumorfrei & \\
\hline 12 & Urozystitis & Urozystitis & b) & \\
\hline 13 & Ta low grade & Urozystitis follicularis & b) & 3 \\
\hline 14 & Urozystitis nach BCG & Urozystitis nach BCG & b) & \\
\hline 15 & T2 high grade & T2 high grade & a) & \\
\hline 16 & Ta low grade & Ta low grade & tumorfrei & \\
\hline 17 & Ta low grade & Ta low grade & 3+9 Uhr positiv & 4 \\
\hline 18 & Ta low grade & Ta low grade & $\begin{array}{l}\text { Nachresektion } \\
\text { positiv }\end{array}$ & 5 \\
\hline 19 & Ta low grade & Ta low grade & tumorfrei & \\
\hline 20 & T1 high grade + Cis & $\begin{array}{l}\text { T1 high grade } \\
+ \text { Cis }\end{array}$ & a) & \\
\hline 21 & vager Cis Verdacht & Urozystitis & b) & 6 \\
\hline 22 & Cis & Cis & c) & \\
\hline 23 & Ta high grade & $\begin{array}{l}\text { T1 high grade } \\
+ \text { Cis }\end{array}$ & $\begin{array}{l}\text { Randproben } \\
\text { positiv }\end{array}$ & 7 \\
\hline 24 & $\mathrm{TaG} 2$ & $\mathrm{TaG} 2$ & tumorfrei & \\
\hline
\end{tabular}


7) Stark koagelbelegter Tumor, somit schwierige Beurteilung des teils soliden Tumors. Großkalibriges zuführendes Gefäß, unregelmäßiger Gefäßverlauf. Das Gefäß ist stark kontrastreich und liegt oberflächlich, deshalb wird ein Ta-Stadium prognostiziert. Anhand der soliden Tumorkonfiguration wäre retrospektiv die Vorhersage eines T1-Stadiums genauso möglich.

8) Der Operateur beschreibt einen gering suspekte Schleimhautaufwölbung, obwohl von einer Blutauflagerung ausgegangen wird. In Narkose war durch den vagen Tumorverdacht eine Sicherheitsresektion angezeigt, auch wenn sich dieser nicht bestätigte. 


\subsection{Untersuchungsergebnisse für Weißlicht und PDD}

Tabelle 7 zeigt die Ergebnisse bzgl. Tumordetektion und Tumorrandbestimmung für Weißlicht und PDD. Der Vergleich der Ergebnisse findet in den jeweiligen Abschnitten zu NBI statt. Die Diskussion erfolgt unter 5 Diskussion.

Tabelle 7: Ergebnisse Weißlicht- und PDD-Resektion in Tumordetektion, Unterscheidung benigner Befunde und

\begin{tabular}{|c|c|c|c|c|c|c|}
\hline $\begin{array}{l}\text { Pat. } \\
\text { Nr. }\end{array}$ & $\begin{array}{l}\text { WL- } \\
\text { Tumordetekt }\end{array}$ & $\begin{array}{l}\text { WL- } \\
\text { Zystitis? }\end{array}$ & $\begin{array}{l}\text { WL- } \\
\text { Tumorgren- } \\
\text { zen }\end{array}$ & $\begin{array}{l}\text { PDD- } \\
\text { Tumordetekt }\end{array}$ & $\begin{array}{l}\text { PDD- } \\
\text { Zystitis? }\end{array}$ & $\begin{array}{l}\text { PDD- } \\
\text { Tumorgren- } \\
\text { zen }\end{array}$ \\
\hline 1 & + & $\mathbf{E}$ & + & + & $\mathbf{E}$ & - \\
\hline 2 & + & $\mathbf{E}$ & + & + & $\mathbf{E}$ & - \\
\hline 3 & $\mathbf{E}$ & + & $\mathbf{E}$ & $\mathbf{E}$ & + & $\mathbf{E}$ \\
\hline 4 & $\mathbf{E}$ & + & $\mathbf{E}$ & $\mathbf{E}$ & - & $\mathbf{E}$ \\
\hline 5 & $\mathbf{E}$ & + & $\mathbf{E}$ & $\mathbf{E}$ & + & $\mathbf{E}$ \\
\hline 6 & $\mathbf{E}$ & + & $\mathbf{E}$ & $\mathbf{E}$ & + & $\mathbf{E}$ \\
\hline 7 & $\mathbf{E}$ & + & $\mathbf{E}$ & $\mathbf{E}$ & - & $\mathbf{E}$ \\
\hline 8 & + & $\mathbf{E}$ & + & + & $\mathbf{E}$ & + \\
\hline 9 & $\mathbf{E}$ & + & $\mathbf{E}$ & $\mathbf{E}$ & - & $\mathbf{E}$ \\
\hline 10 & + & $\mathbf{E}$ & + & + & $\mathbf{E}$ & + \\
\hline 11 & + & $\mathbf{E}$ & + & + & $\mathbf{E}$ & + \\
\hline 12 & $\mathbf{E}$ & + & $\mathbf{E}$ & $\mathbf{E}$ & + & $\mathbf{E}$ \\
\hline 13 & $\mathbf{E}$ & + & $\mathbf{E}$ & $\mathbf{E}$ & - & $\mathbf{E}$ \\
\hline 14 & $\mathbf{E}$ & + & $\mathbf{E}$ & $\mathbf{E}$ & - & $\mathbf{E}$ \\
\hline 15 & + & $\mathbf{E}$ & + & + & $\mathbf{E}$ & + \\
\hline 16 & + & $\mathbf{E}$ & + & + & $\mathbf{E}$ & + \\
\hline 17 & + & $\mathbf{E}$ & - & + & $\mathbf{E}$ & - \\
\hline 18 & + & $\mathbf{E}$ & + & + & $\mathbf{E}$ & + \\
\hline 19 & + & $\mathbf{E}$ & + & + & $\mathbf{E}$ & + \\
\hline 20 & + & $\mathbf{E}$ & + & + & $\mathbf{E}$ & + \\
\hline 21 & $\mathbf{E}$ & + & $\mathbf{E}$ & $\mathbf{E}$ & + & $\mathbf{E}$ \\
\hline 22 & + & $\mathbf{E}$ & - & + & $\mathbf{E}$ & - \\
\hline 23 & - & $\mathbf{E}$ & - & + & $\mathbf{E}$ & - \\
\hline 24 & + & $\mathbf{E}$ & + & + & $\mathbf{E}$ & + \\
\hline 25 & $\mathbf{E}$ & - & $\mathbf{E}$ & $\mathbf{E}$ & + & $\mathbf{E}$ \\
\hline 26 & $\mathbf{E}$ & + & $\mathbf{E}$ & $\mathbf{E}$ & + & $\mathbf{E}$ \\
\hline
\end{tabular}

- bei einer Urozystitis entfällt (E) die Tumorgrenzenbestimmung und die Tumordetektionsangabe aufgrund der Benignität

- bei einer Tumordetektion entfällt (E) die Angabe zur Urozystitis.

- ein „,+“ steht für eine korrekte Einschätzung, ein „,-“dagegen für eine Fehleinschätzung (bzgl. Tumorrand und Detektion) 


\subsection{Präzision der Tumordetektion}

○ Können alle tumorsuspekten Areale, die in Weißlicht- oder der PDD-Technik detektiert werden auch in der NBI-Technik gefunden werden?

In der NBI-Technik wurden keine Tumoren übersehen, die in anderen Bildtechniken detektiert worden sind.

○ Ist die NBI-Technik in der Lage, zusätzliche Tumoren zur Weißlicht- oder der PDDTechnik zu detektieren?

In der NBI-Technik konnte im Vergleich zur Weißlichttechnik ein zusätzlicher Tumor detektiert werden. Die PDD-Tumordetektion stimmte mit der Detektion in NBI überein. 


\subsection{Präzision der Dignitäts- und Stadienvorhersage}

○ Können in der NBI-Technik nicht-maligne Tumoren sicher von malignen Tumoren unterschieden werden?

Zusammengefasst können nicht maligne von malignen Tumoren mit einer hohen, trotzdem nicht ausreichenden Sicherheit durch die NBI-Technik unterschieden werden, allerdings mit dem Risiko einer falsch-positiven Bewertung. Unter den Aspekten der Patientensicherheit ist die Probenentnahme bei unklarer Dignität mit dem Risiko einer falsch positiven Bewertung jedoch unvermeidlich. Bei der Festlegung auf sicher benigne Befunde (richtig negativ) besteht ein zu hohes Risiko für einen unbehandelten Tumor (mit gleichzeitigem Risiko für eine Progression)

\section{○ Ist in der NBI-Technik eine Stadiendifferenzierung des Blasenkarzinoms möglich?}

Ist in der Histologie ein Tumor nachgewiesen worden, stimmte die NBI-Stadienprognose in 13/14 Fällen (= 92,9\%) (26 Tumoren, davon 14 maligne) überein.

Es konnte eine akkurate Unterscheidung zwischen low grade und high grade-Läsionen anhand des entwickelten Schemas und der nachfolgenden Ausführungen erfolgen. Bei infiltrierenden Tumoren ( $n=2)$ ergaben sich bei keinem der zwei Patienten positive Absetzungsränder in der Resektionstiefe, jedoch gelang es auch bei keinem Patienten, einen Aspekt für eine sichere Beurteilung zu eruieren. Zur Interpretation der Ergebnisse s. Absatz 5 Diskussion 


\subsection{Präzision der Ausdehnungsbestimmung des Blasenkarzinoms}

○ Ist eine genaue Beurteilung des Tumorrandes, also des genauen Übergangs von pathologischem zu gesundem Gewebe möglich?

Zur Beurteilung der Präzision der NBI-Technik in der Tumorausdehnung wurden Randproben direkt an der in der NBI-Technik festgelegten Grenze zwischen tumorverändertem Gewebe und sicher gesundem Gewebe entnommen. Das Sicherheitsmarginal im Rahmen der Tumorresektion wurde bewusst knapp gehalten (ca. $2 \mathrm{~mm}$ ) um eine möglichst genaue Aussage über eine korrekte oder inkorrekte Tumorrandbeurteilung zu erhalten. Es fanden sich bei 10/14 Patienten (71,4 \%) tumorfreie Randproben.

Bei 4/14 Randproben zeigte die histologische Untersuchung trotz NBI-makroskopischer Tumorfreiheit also ein residuales Tumorgeschehen.

Zusammengefasst ist eine Beurteilung des Tumorrandes in unserem Patientenklientel nur mit mäßiger Sicherheit gelungen. Die Infiltrationstiefe konnte nicht mit ausreichender Sicherheit eingeschätzt werden. 


\subsection{Beispielbilder}

\subsubsection{Urozytitis}
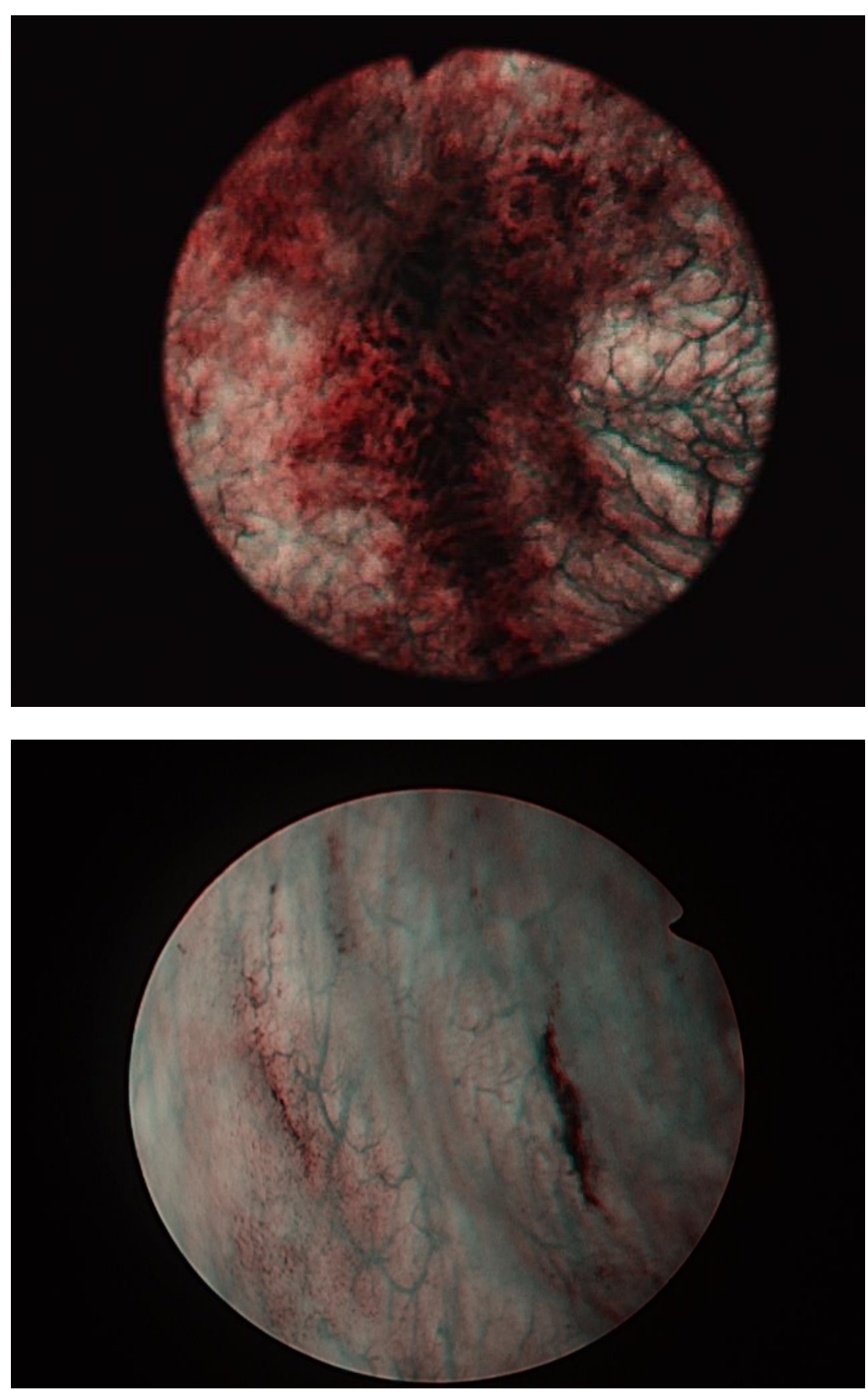

Beide Bilder zeigen eine Urozystits. Ein exophytischer Tumor kann ausgeschlossen werden. Ein Carcinoma in situ-Areal demarkiert sich augenscheinlich nicht. Es handelt sich um eine Urozystitis mit Blutauflagerungen (sehr kontrastreich) und feinen Kapillaren. 


\section{Urozystitis}
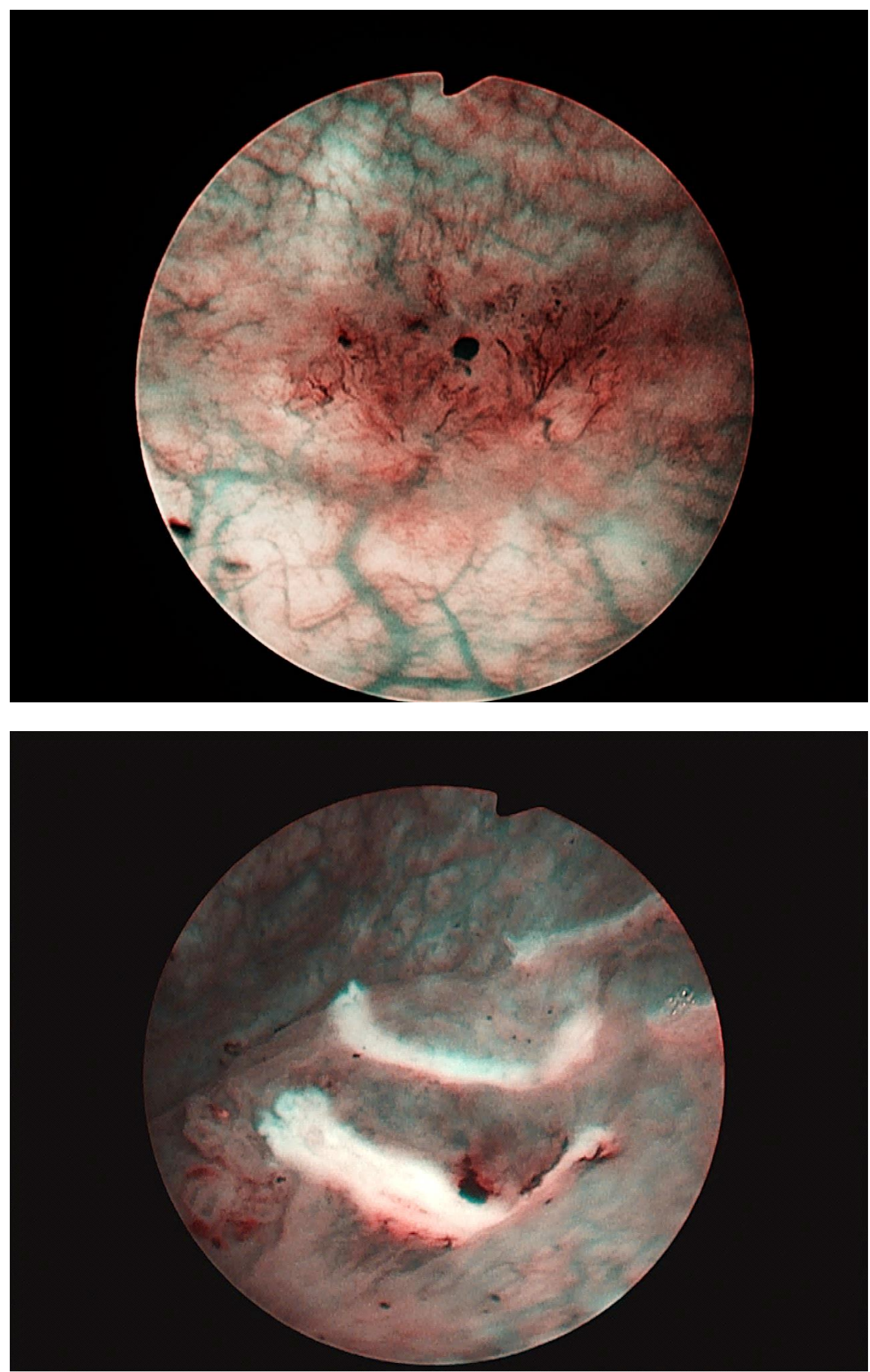

Chronisch rezidivierende Urozystitis. Es sind zwar papilläre Anteile erkennbar, doch in NBI gut zu sehen ist, wie das Gefäß im papillären Anteil von viel Schleimhaut ausgekleidet wird.

Bild unten: Markierung durch Resektionsschlingenabdruck 


\section{Urozystitis}

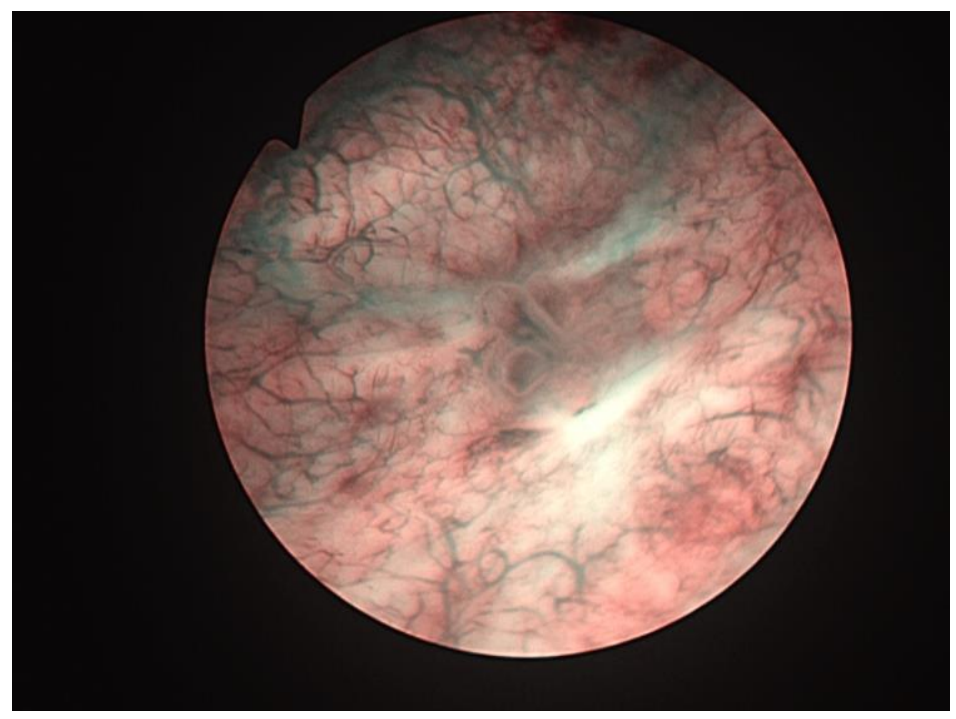

Der papilläre „Tumor“ besitzt keine Malignität, auch hier ist der Gefäßanteil im Tumor sehr gering, die Schleimhaut scheint in NBI lediglich aufgeworfen. Die Gefäßzeichnung ist feinkapillär.
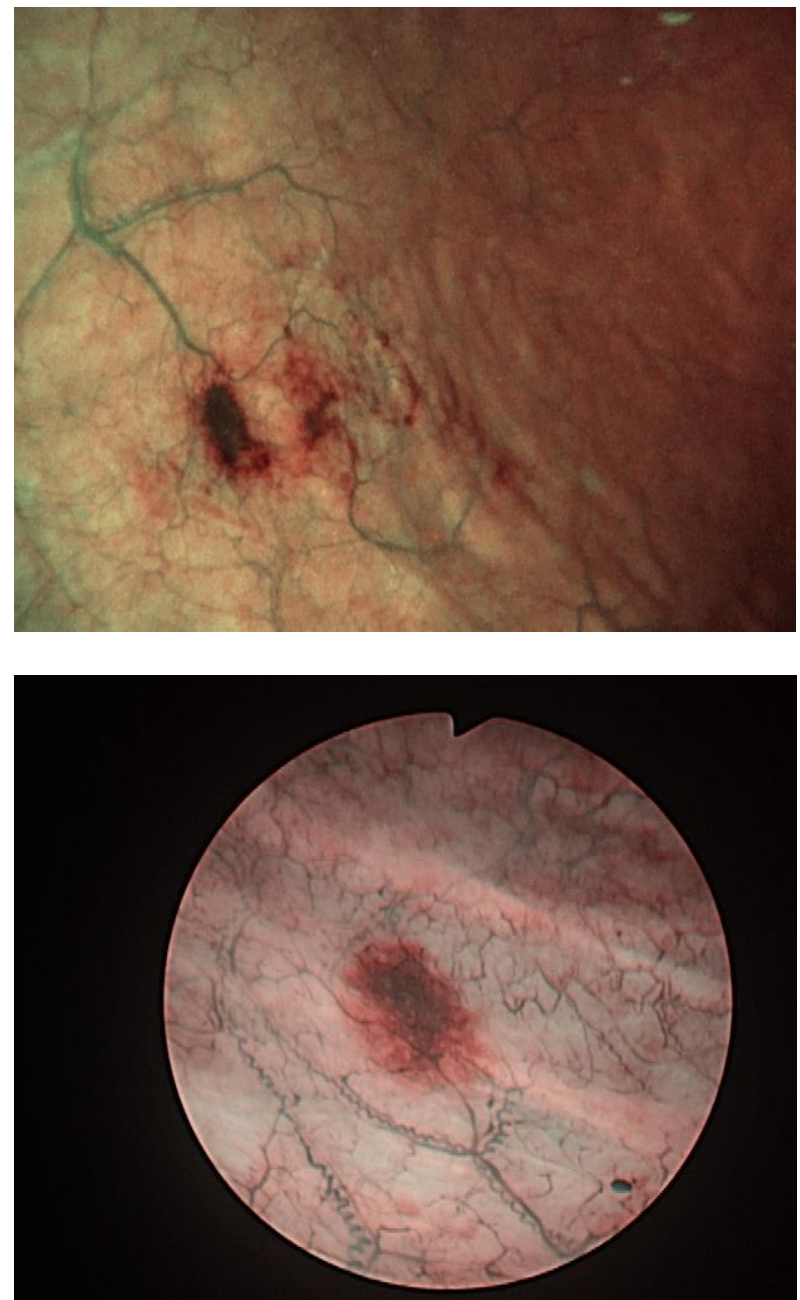

Die demarkierten Areale zeigen einen starken Kontrast (unten ein Bild in HD-Auflösung). Dies entspricht Blutauflagerungen. Durch die Lichtabsorption direkt an der Oberfläche ist dies sehr imponierend. Entsprechend ist eine Blutauflagerung von einem weniger kontrastreichen Cis zu unterscheiden, da dies erst unter der Schleimhaut Licht absorbiert und reflektiert. 


\subsubsection{Tumorstadium TaG1}

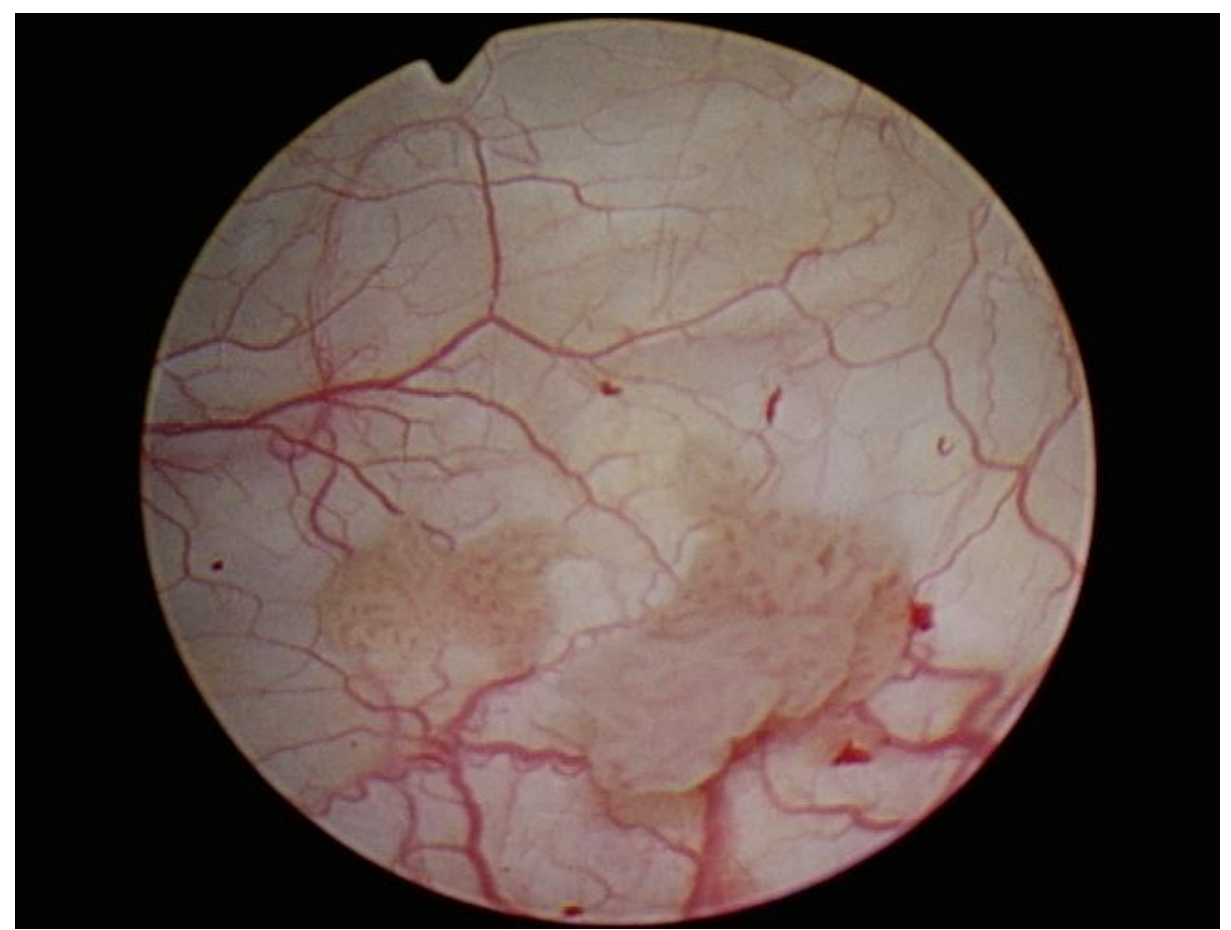

Im Vergleich zu den „Tumoren“ unter 4.8.1 Urozytitis erscheinen die low grade Karzinome TaG1 etwas gefäßreicher und nahezu immer exophytisch. Die Gefäße erscheinen retikulär, es gibt jedoch beginnend dezente Kaliberunterschiede.

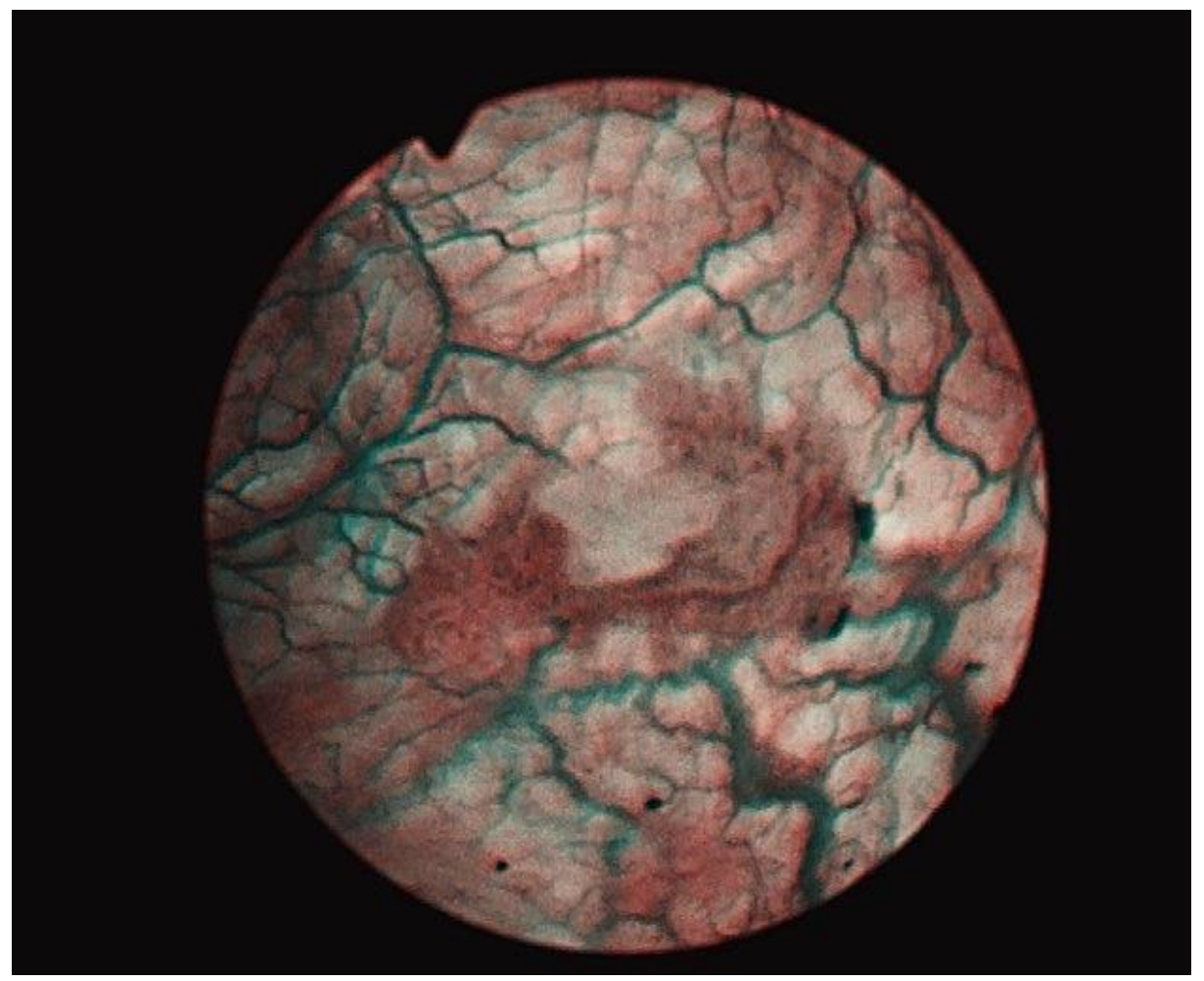




\section{Tumorstadium TaG1}
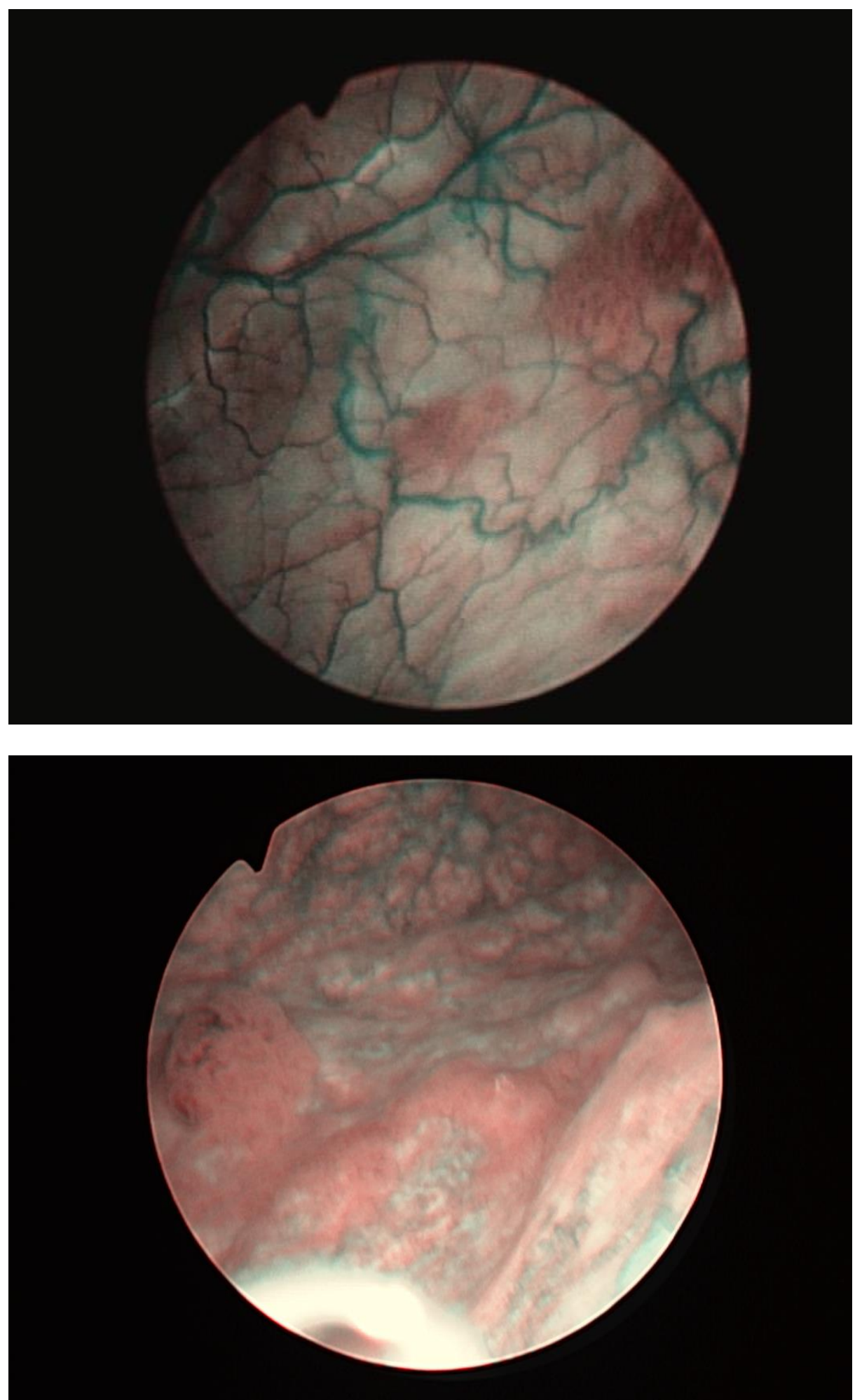

Papillärer, invertierter Tumor im Stadium TaG1. Die Differenzierung zur Urozystitis ist aufgrund des niedrigen Gefäßanteils zuungunsten eines höheren Schleimhautanteils im Tumor schwierig. Der Übergang zwischen benigner Läsion und maligner Läsion ist also sehr fließend. 


\subsubsection{Tumostadium TaG2 low grade}
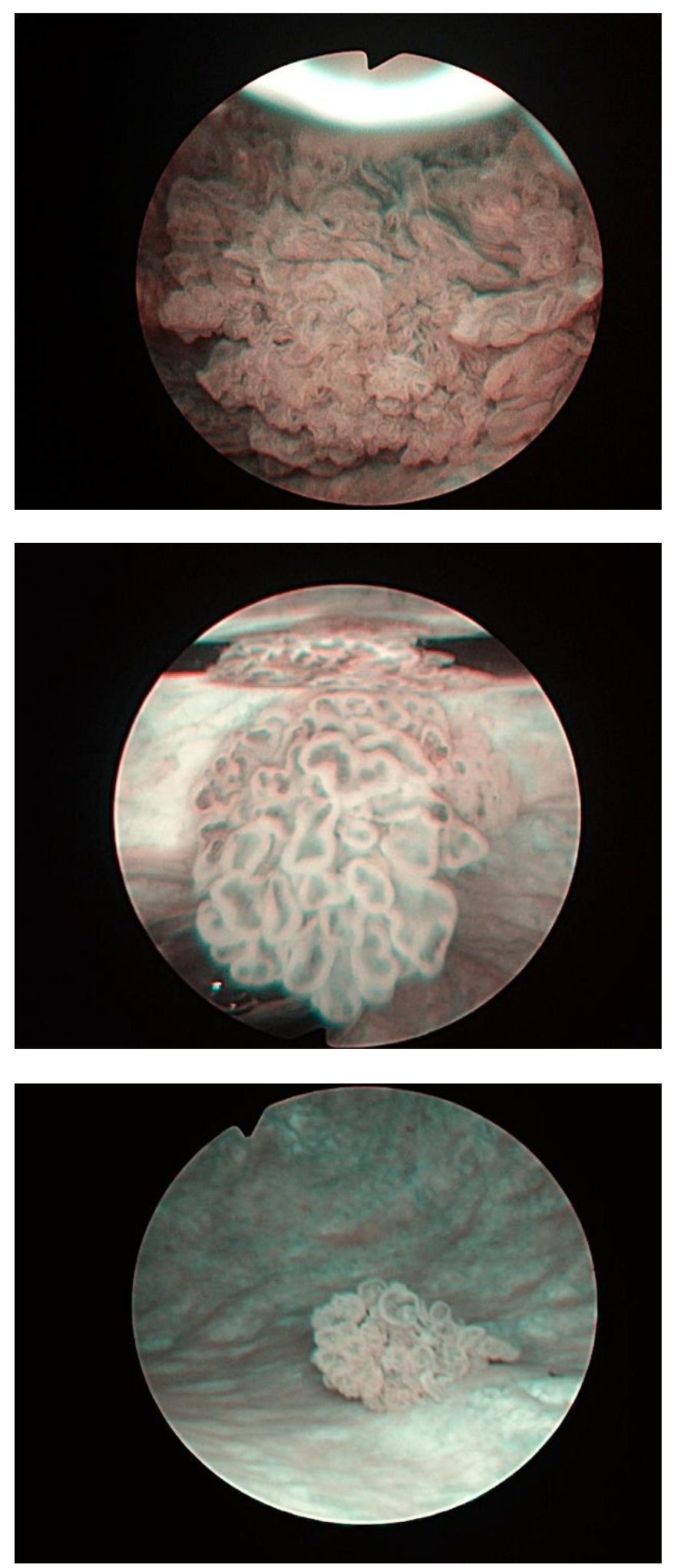

Typische TaG2 Befunde mit papillärem, exophytischem Tumor und den einsprießenden Gefäßen. Insgesamt sind Parallelen zwischen den TaG2-Befunden sichtbar. Mäßig hoher Anteil an Gefäßen im Tumor. 
Tumostadium TaG2 low grade
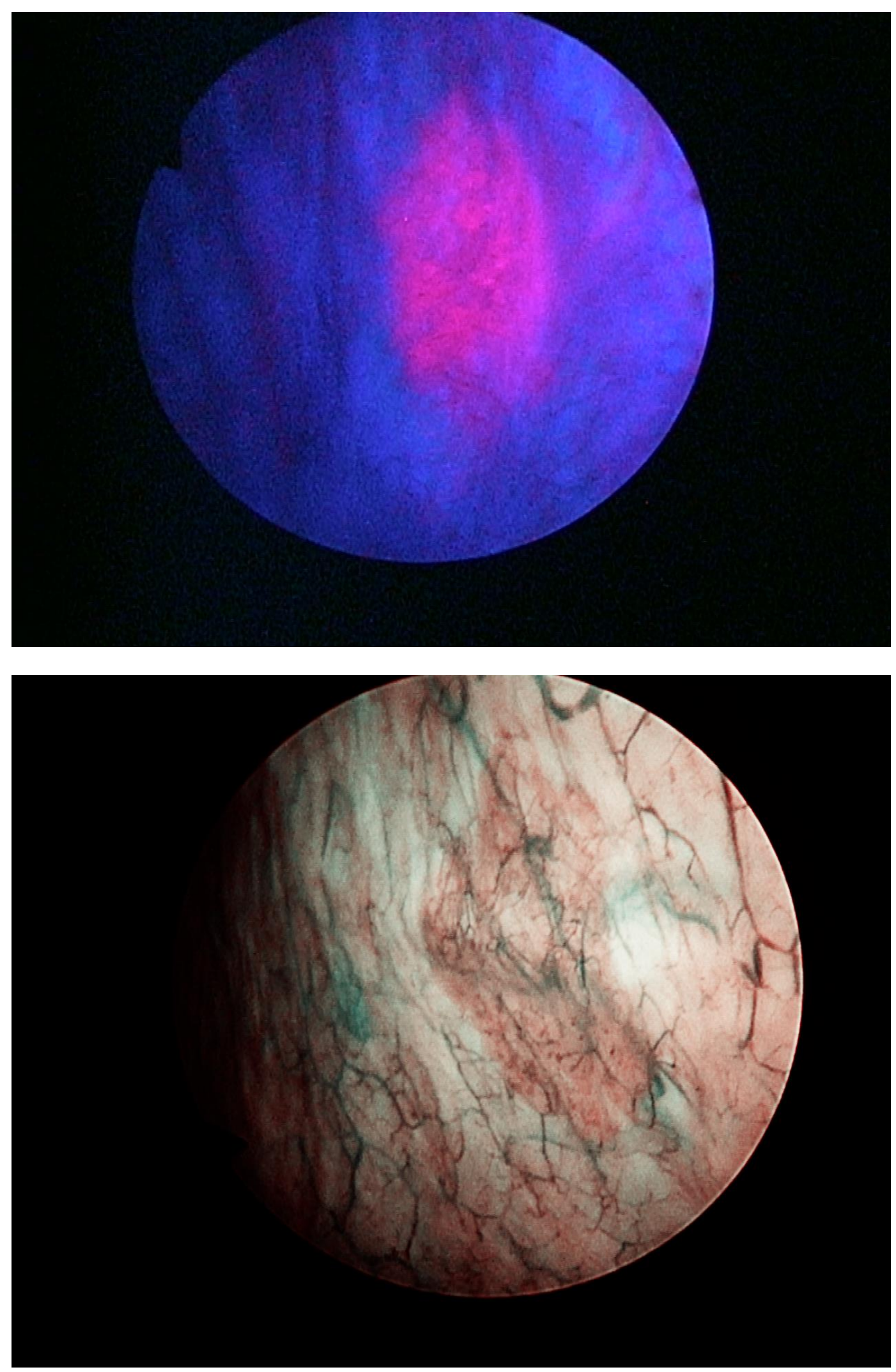

Papillärer Rasen, in PDD deutlich fluoreszierend, in PDD werden die papillären Strukturen diesmal besser erkannt, während die NBI-Technik auch ein Cis vermuten lassen könnte. 


\subsubsection{Tumorstadium TaG3}
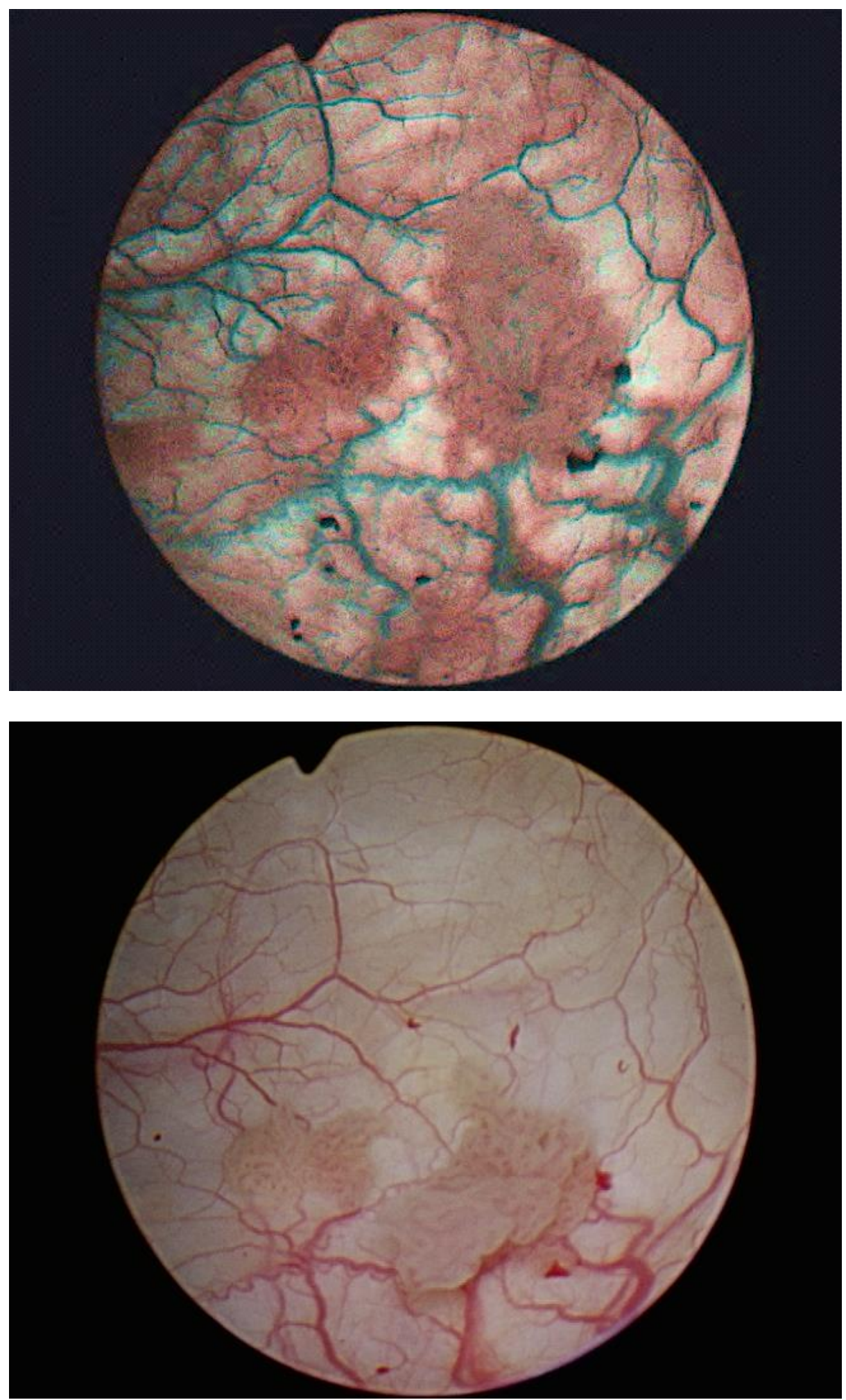

Die Gefäßanteile im Tumor, hier auch in Weißlicht gut zu sehen, und die unregelmäßig konfigurierten, teils kaliberstarken (Kalibersprünge) zuführenden Gefäße lassen hier eine schlechtere Differenzierung vermuten. 


\subsubsection{Tumorstadium T1G2}

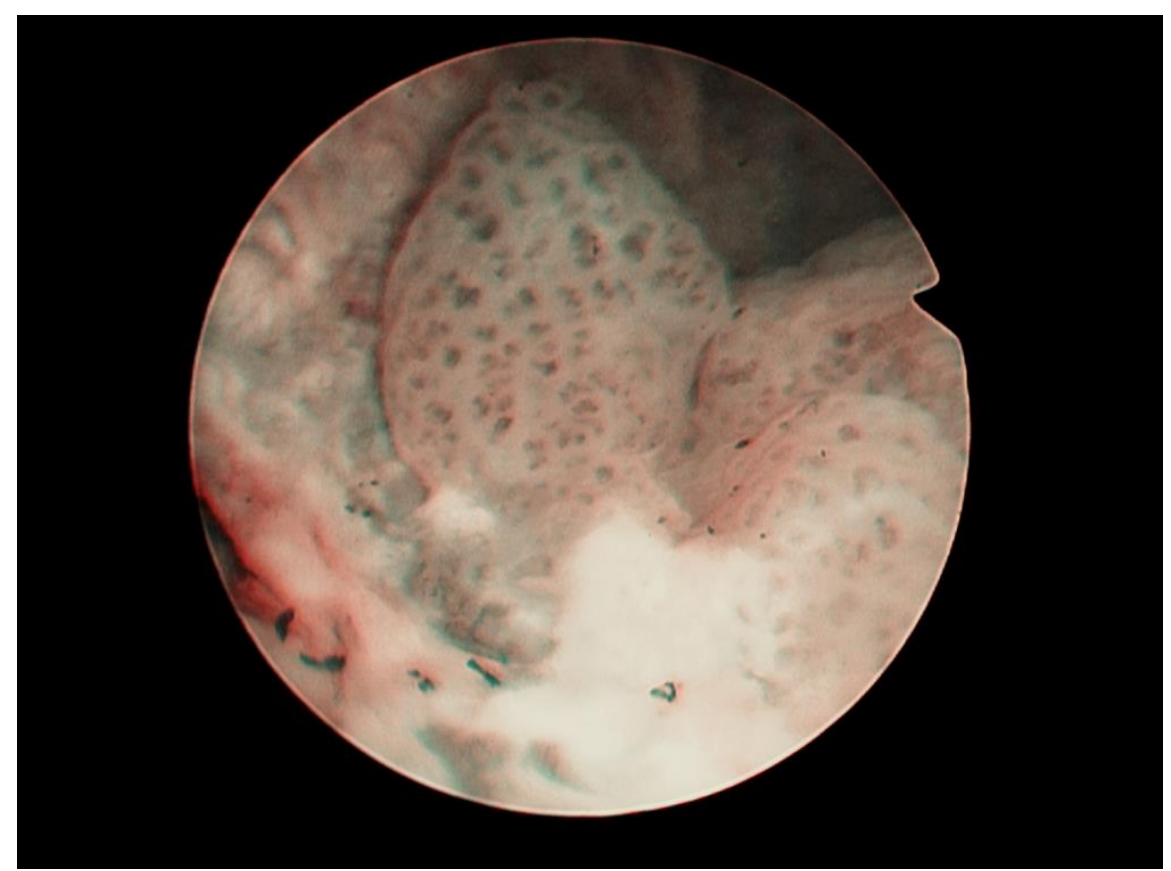

Breitbasiger Tumor, der auch „knollig“ wirkt und basal sehr unregelmäßig erscheint. 


\subsubsection{Tumorstadium T1G3}

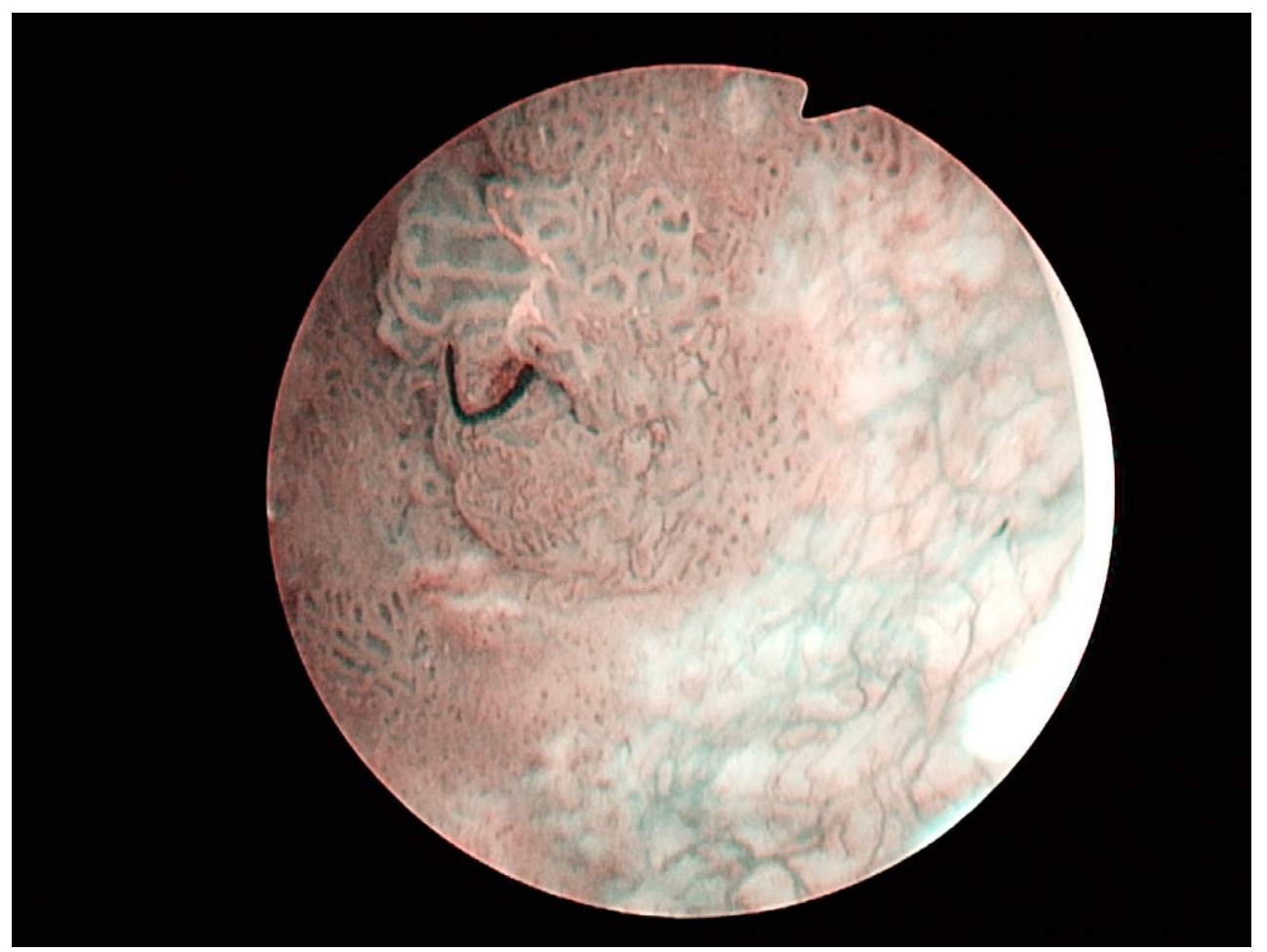

Anmerkung: Hier ist in drei Randproben 3, 5 und $12 \mathrm{Uhr}$ Carcinoma in situ nachgewiesen worden. Die Randproben sind - hier gut zu sehen - vom Übergang des papillär exophytischen Tumoranteils in die Peripherie entnommen worden. Diese Peripherie, in Weißlicht gänzlich unauffällig, erscheint „rötlichfleckiger" als gewöhnlich. Zudem ist dem exophytischen Tumoranteil hier eine grünliche Randumgebung anliegend, die sich von der rosigen Schleimhaut (vermutlich normales Urothel) weiter entfernten Peripherie demarkiert. In den differenzierenden Resektionen werden eben jene auffälligen Randbereiche isoliert entnommen und getrennt eingesandt. So war es im Nachhinein möglich zu sagen, dass der Haupttumor bei subepithelialem Wachstum Anteile einer G2- und einer G3- Differenzierung hat, während der angrenzende Bereich histologisch Cis-Anteile enthält. 


\section{Tumorstadium T1G3}
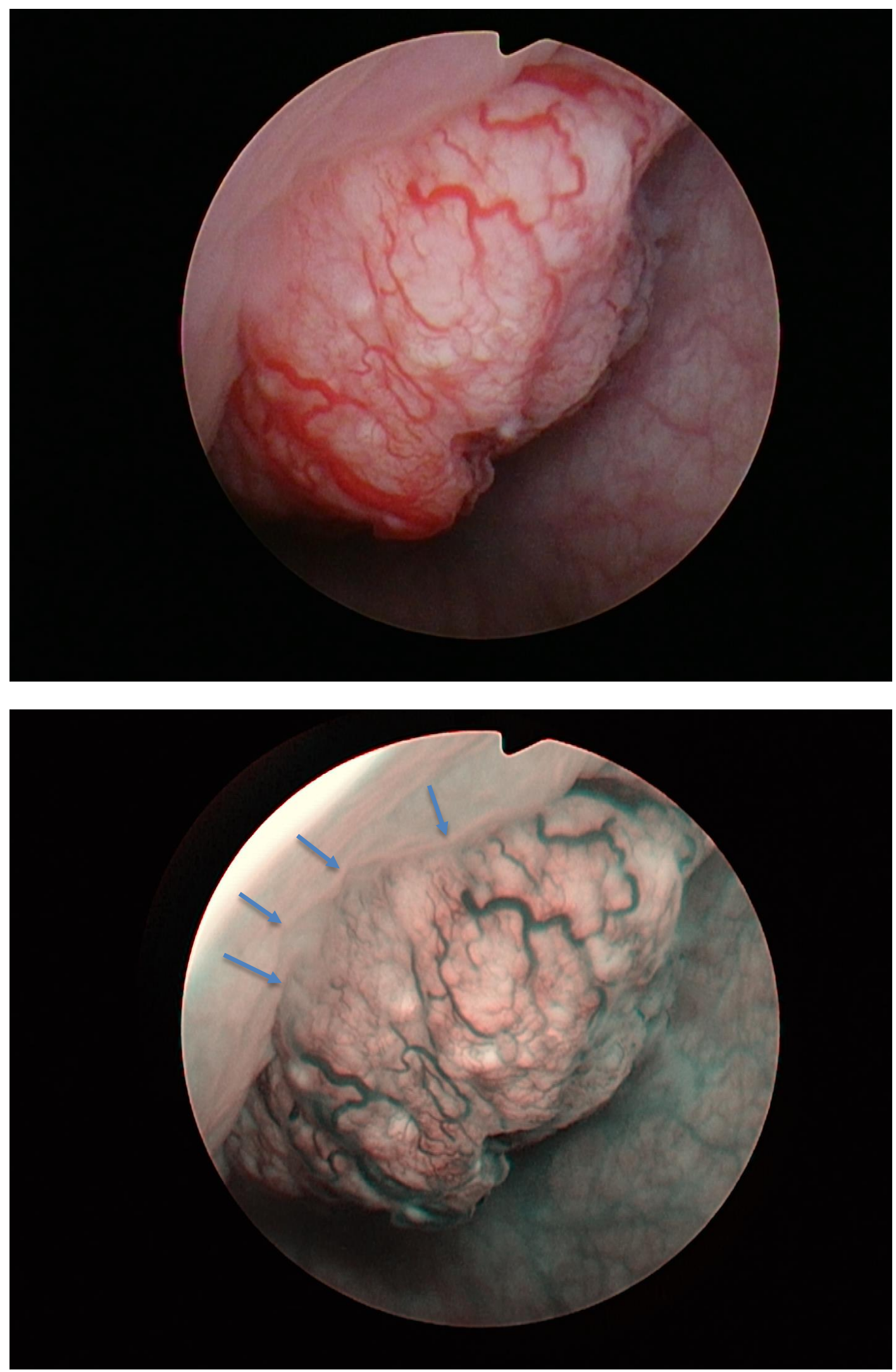

Eindrucksvolle Darstellung der Unterschiede von Weißlicht und Cis. In beiden Bildtechniken ist der Tumor gut zu erkennen. In NBI genaue Festlegung des Tumorrandes (Pfeile). 


\section{Tumorstadium T1G3}


Gleiche Ansicht auf gleiches Tumorareal in allen Bildtechniken. In NBI sieht man, dass die Gefäße sehr unregelmäßig im Verlauf und zudem unterschiedlich im Kaliber sind. Sowohl dieser Aspekt als auch die unterschiedliche Farbintensität sind im NBI eher hinweisgebend auf ein weiter fortgeschrittenes, tieferes Tumorwachstum und ein schlechteres Grading. Die unterschiedliche Farbgebung ist dabei jedoch kein sicheres Zeichen.

Histologisch sind -vor allem in den Randproben- auch Anteile eines Cis gesichert worden. 


\section{Tumorstadium T1G3}
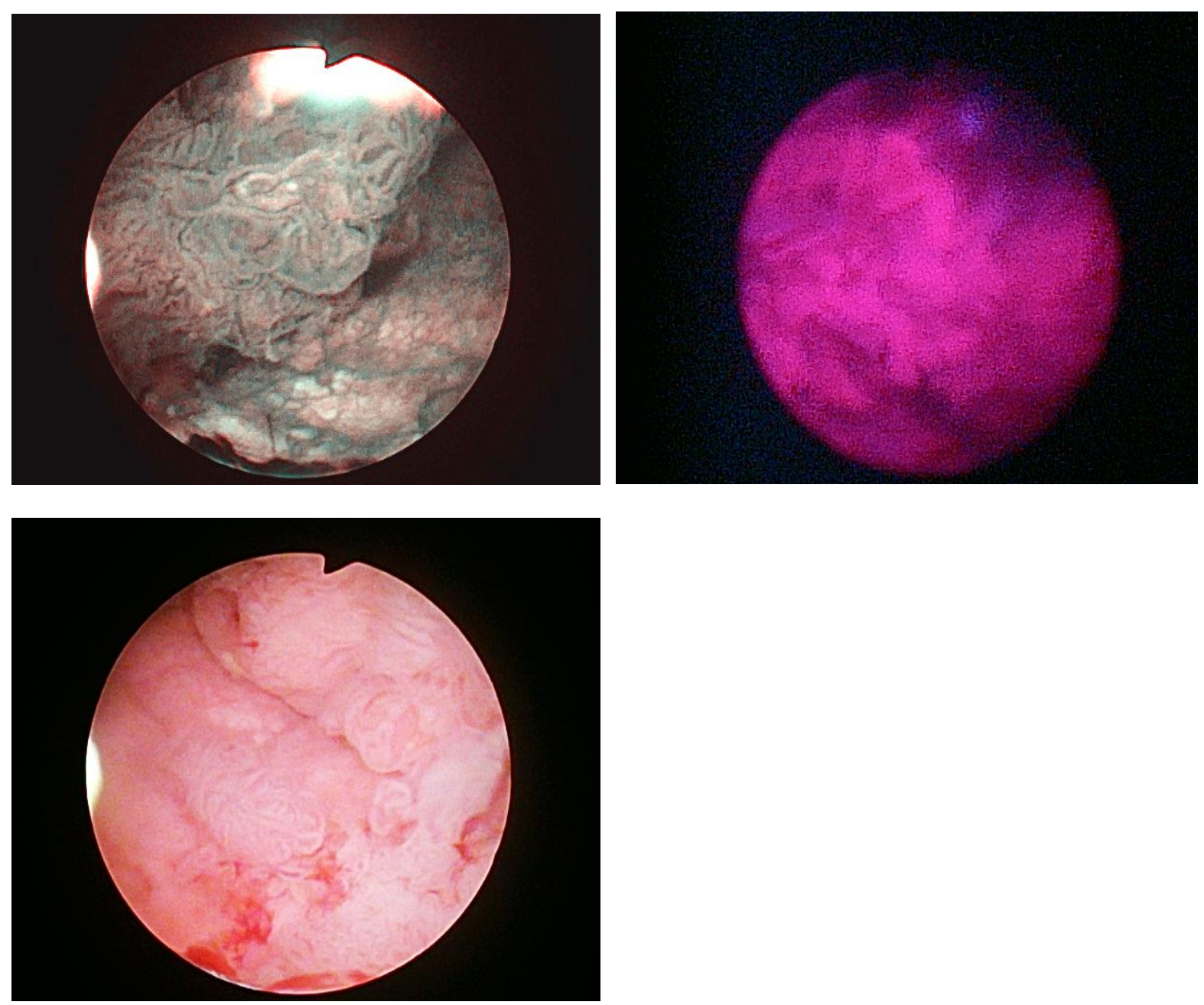

Auch ein T1G3 Tumor. Ein geringer ,glasiger“ Schleimhautanteil zuungunsten einer soliden Formation. Der Übergang zum normalen Blasengewebe ist besonders in NBI scharf abgrenzbar. In PDD fluoresziert der Tumor stark, jedoch sind auch die hypervaskularisierten Areale um den papillären Haupttumor fluoreszierend (falsch positiv). Beide Techniken sind jedoch der alleinigen Weißlichttechnik optisch überlegen. Der Tumor ist in Weißlicht schlechter sichtbar, und der Übergang vom Tumor zum normalen Blasengewebe ist verglichen mit den anderen Bildtechniken am undeutlichsten. 


\subsubsection{Tumorstadium T1G3 (+ Carcinoma in situ)}
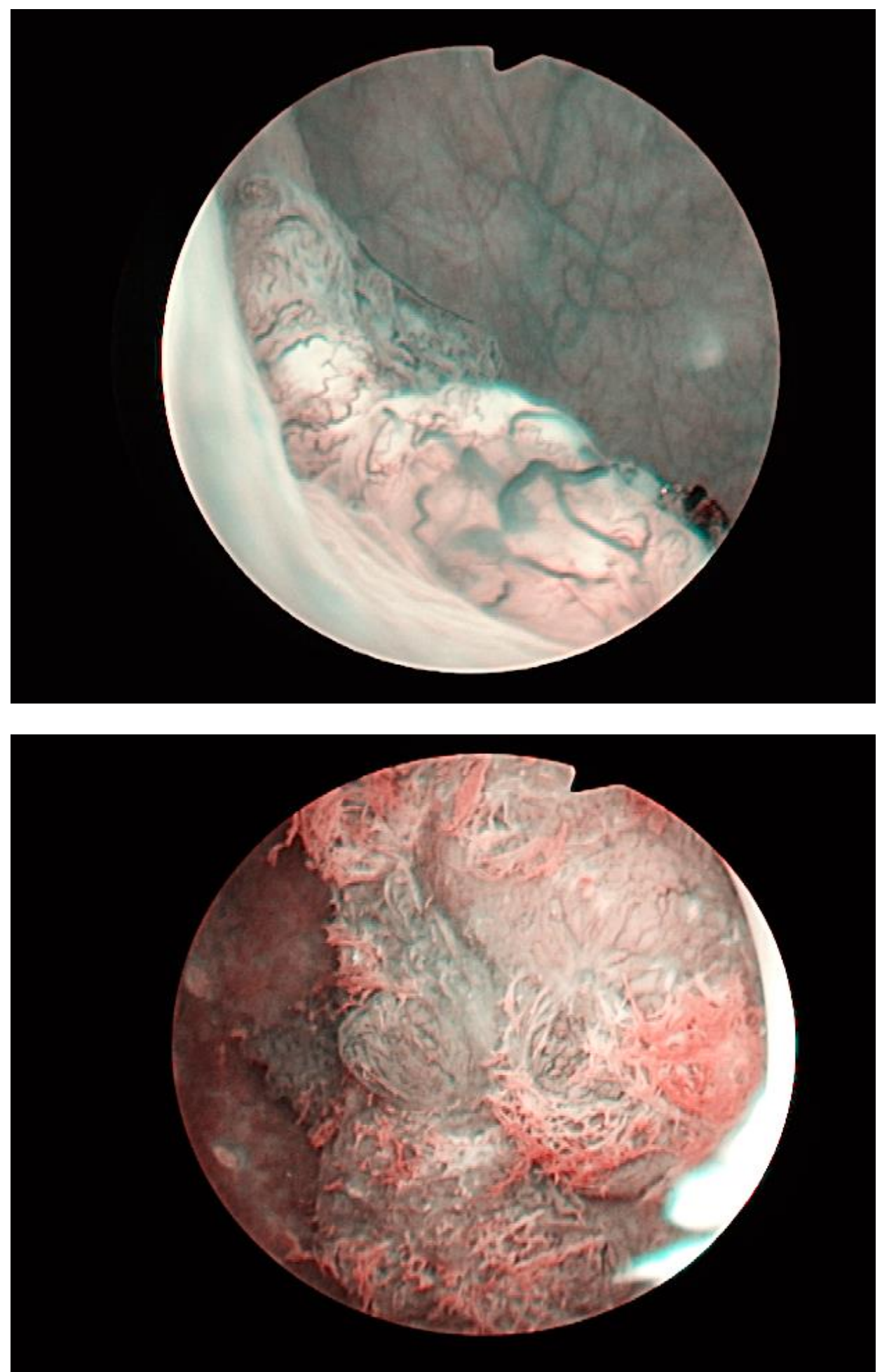

Diese Bilder stammen vom gleichen Patienten. Es sind deutlich die solideren Anteile des als T1G3 klassifizierten Tumors zu sehen. Zusätzlich ergab die Histologie ein Cis (hier nicht dargestellt). 


\section{T1G3+Cis}
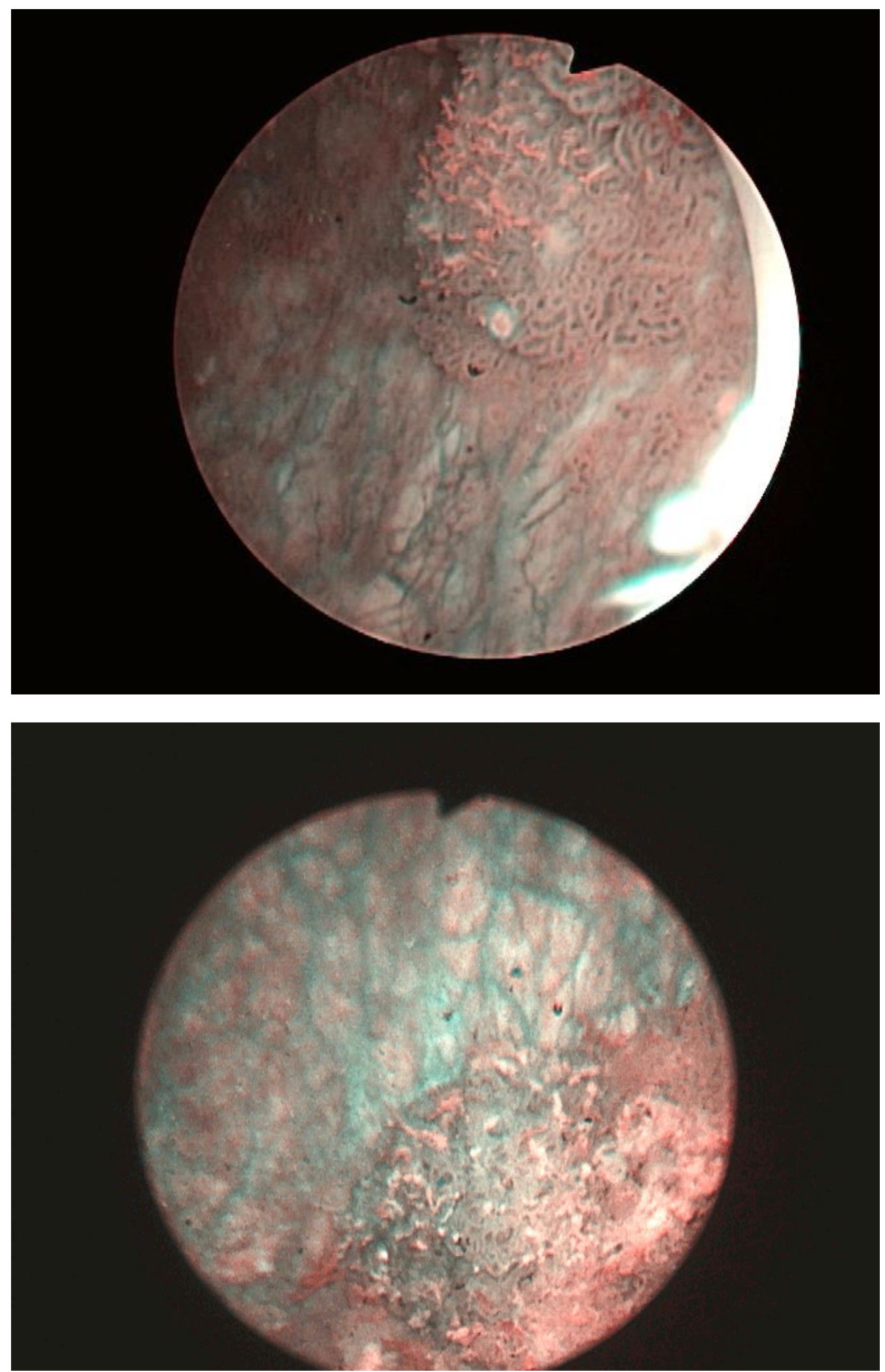

Nochmal der gleiche Patient, hier liegt der Fokus auf den zuführenden Gefäßen. Diese sind im Gegensatz zur Urozystits weniger filiform, sondern deutlich „balkiger“". 


\section{T1G3+Cis}

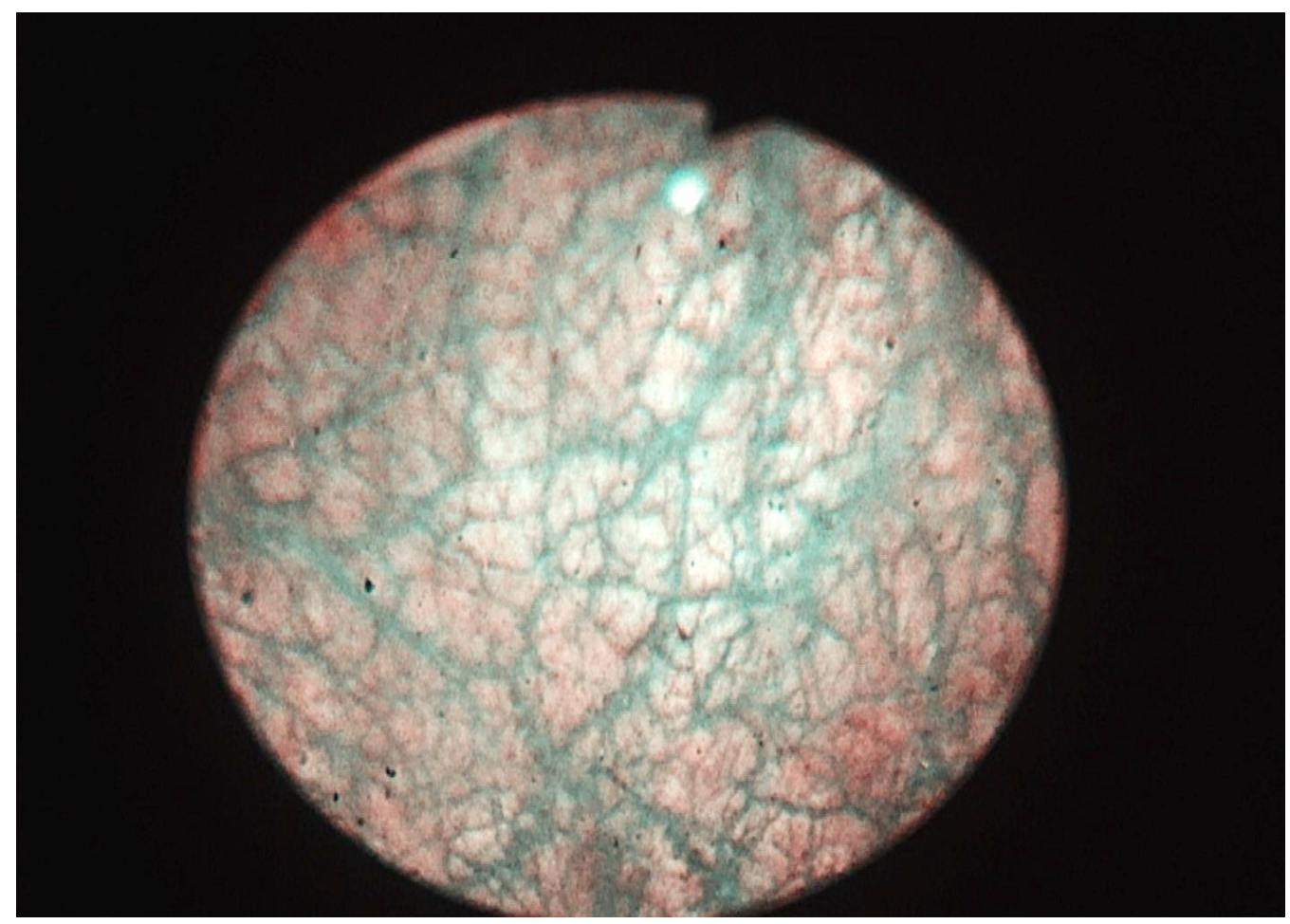

Weiterhin derselbe Patient und Tumor. Der Fokus ist diesmal auf die zuführenden Gefäße gerichtet, am Unterrand des unteren Bildes könnte bereits der histologisch gesicherte Cis Anteil beginnen.



Ein weiterer T1G3-Tumor mit Cis Anteilen. Der Unterschied zu den TaG2-Befunden ist deutlich über den erhöhten Gefäßanteil im verplumpten Haupttumor zu erkennen. 


\subsubsection{Muskelinvasive Tumoren: T2G2 high grade}

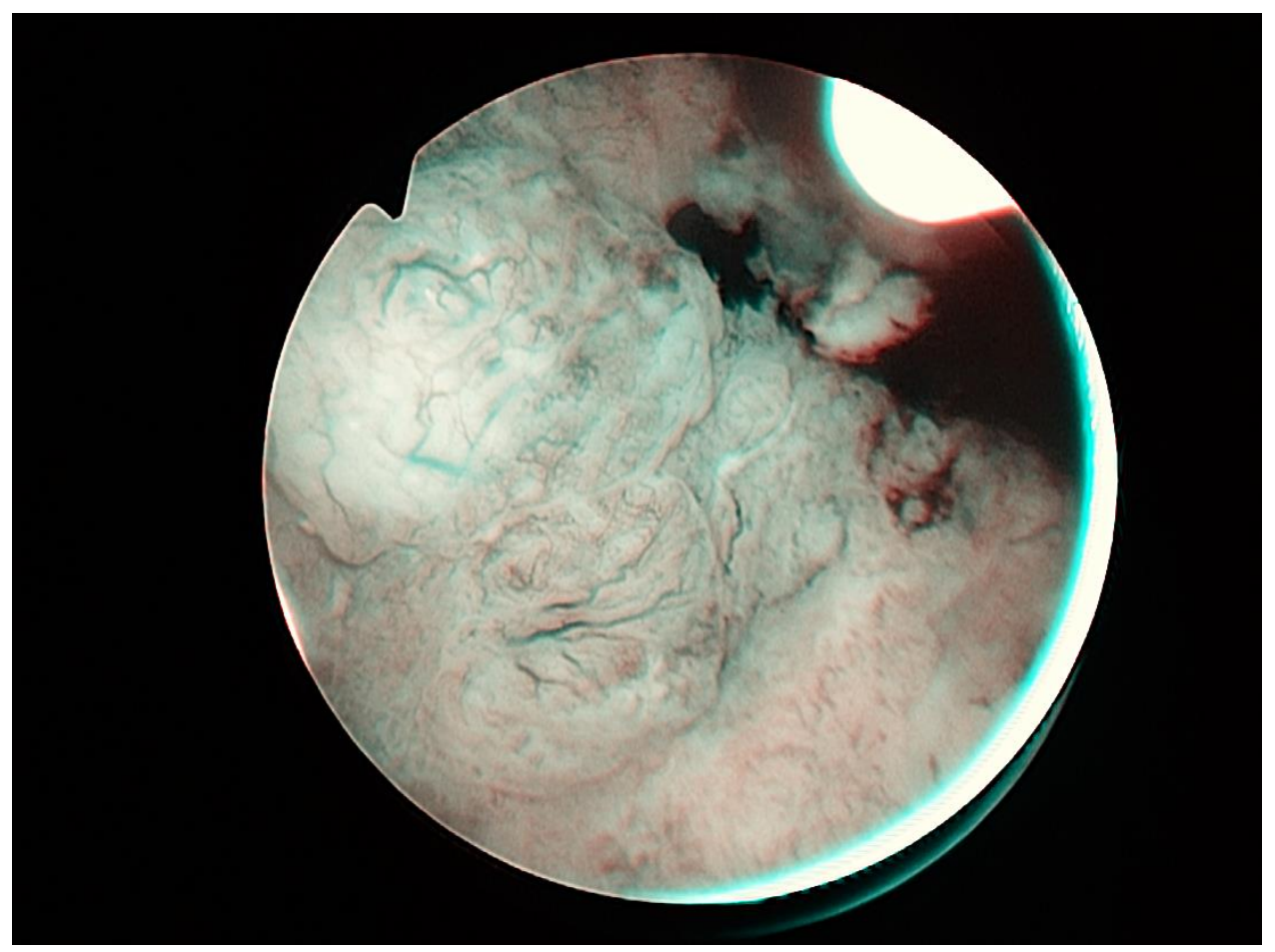

Ein knolliger Tumor im Stadium T2G2. Man erkennt eindeutig die fehlenden Schleimhautanteile, die auf das fortgeschrittene Tumorstadium hindeuten.

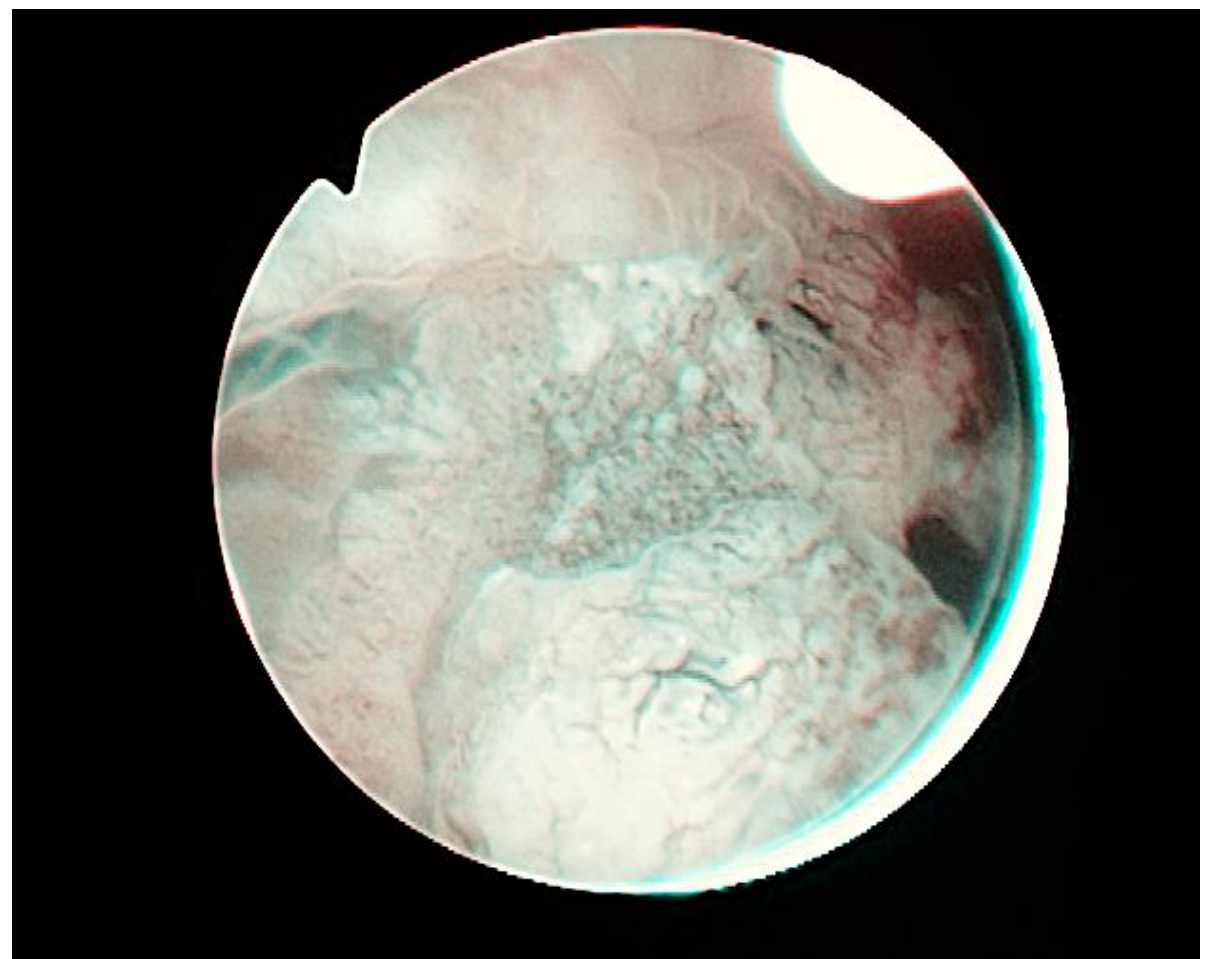

Ein Bild der zuführenden Gefäße des eben gezeigten Tumors. Die balkige, unregelmäßige Struktur passt zum bigh grade-Tumorstadium. 


\subsubsection{Muskelinvasive Tumoren: T2G3}
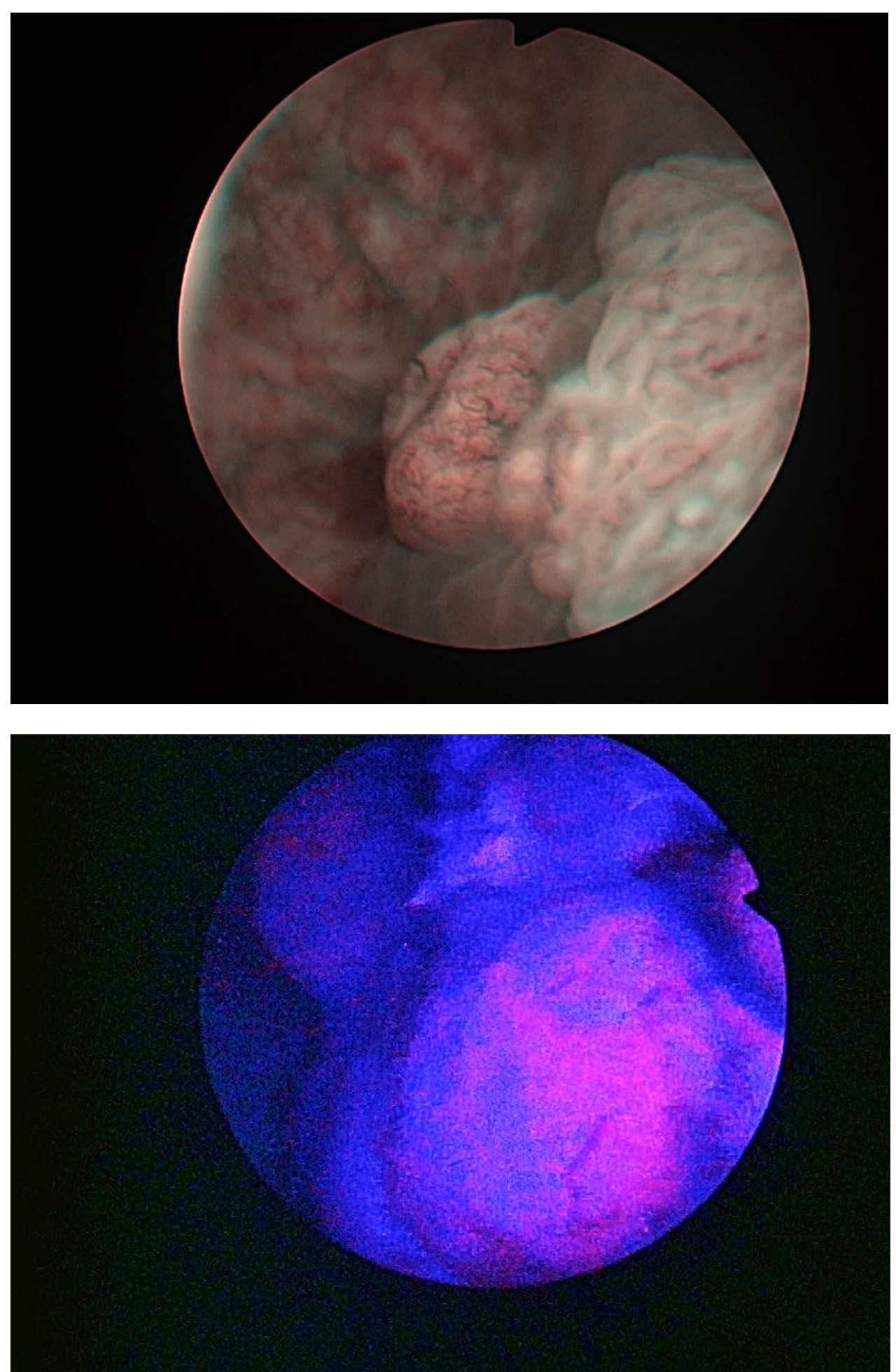

Tumor in Weißlicht (oben) und PDD-Darstellung (unten). Die Fluoreszenz ist nur schwach positiv, aber definitiv pathologisch. Die Hexvix ${ }^{\circledR}$-Lösung hat erfahrungsgemäß Probleme, die gesamte Blase gleichmäßig anzufärben und auszuleuchten. Gerade im Divertikelbereich ist somit eine schwache Anfärbung zu erwarten gewesen. Jedoch könnte auch ein systematischer Fehler vorliegen (siehe 5 Diskussion). Da der Tumor in PDD allein leicht übersehen werden könnte, wird hier deutlich, wie wertvoll eine Kombination der Bildtechniken ist. 
Muskelinvasive Tumoren: T2G3

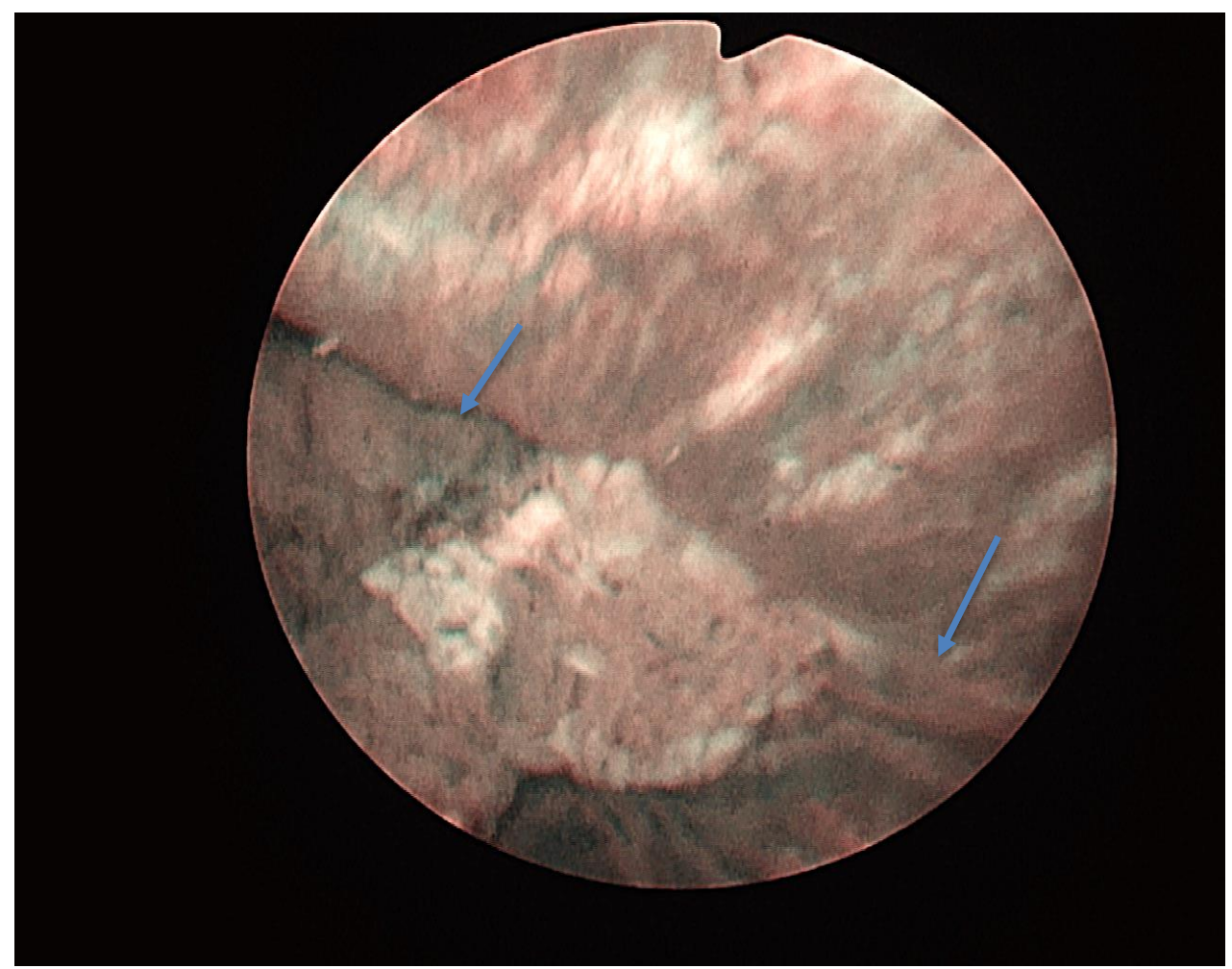

Die Pfeile nach unten kennzeichnen den Divertikelrand, der Tumor liegt tief im Divertikel. Am Bildübergang ist das eigentliche Blasengewebe zu sehen.

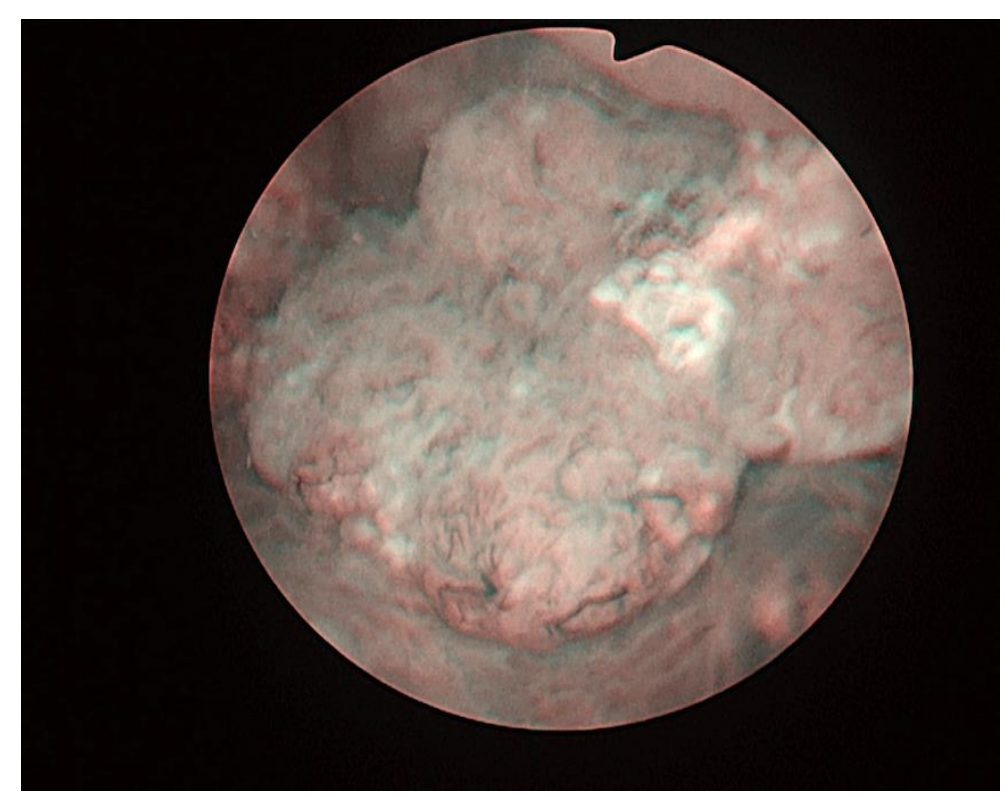

Vier Bilder des gleichen Patienten. Ein in einem Blasendivertikel befindlicher Tumor von schlechter Differenzierung mit Muskelinvasion (T2G3). Man vergleiche hier das Bild gegenüber einem papillären Tumor oder einer Urozystitis. Hier ist der Gefäßanteil im Tumor nicht über $50 \%$, zudem wirkte er knollig und teilweise kalzifizierend (weißliche Struktur). 


\subsubsection{Typische benigne Befunde:}

\subsubsection{Leukoplakie}
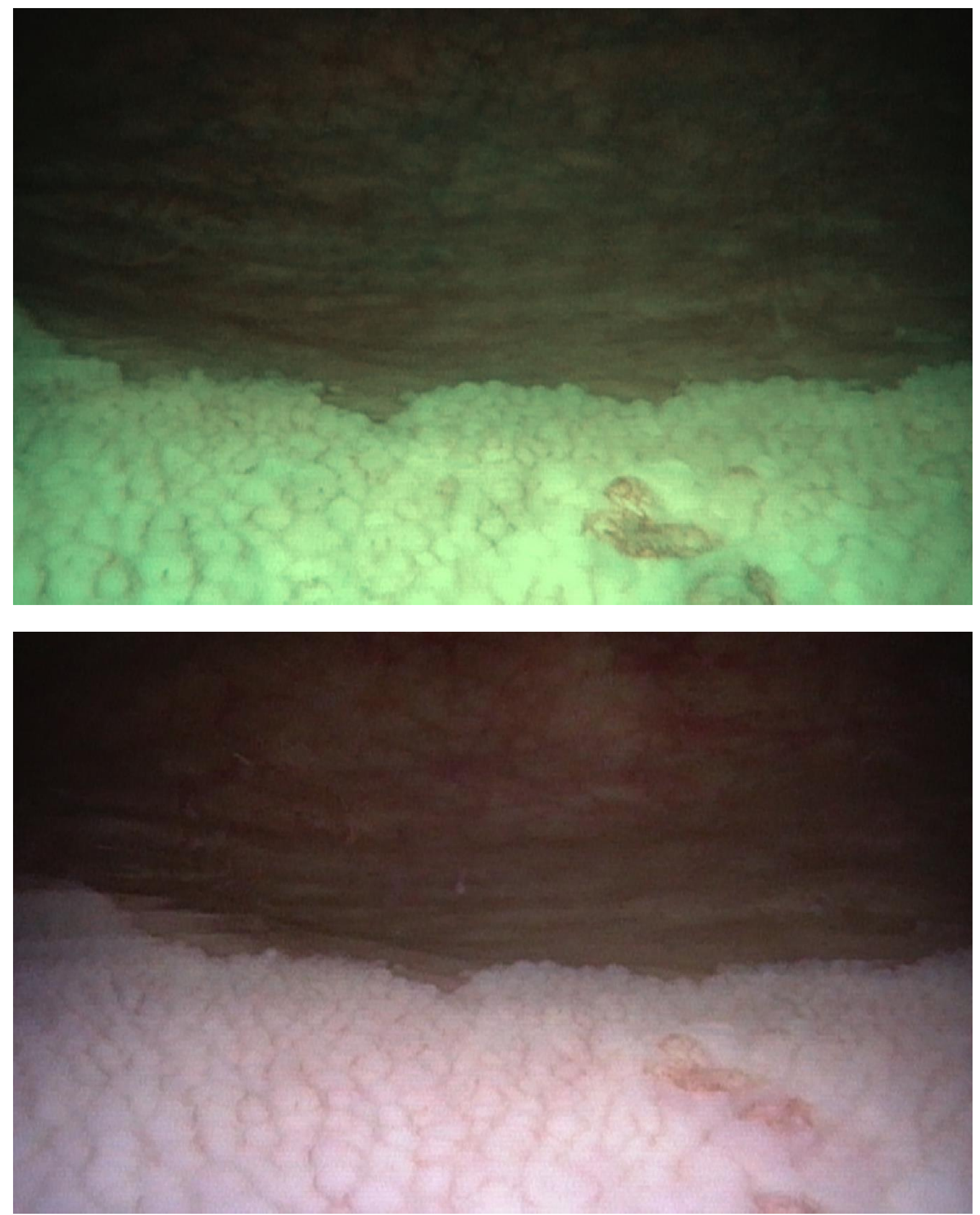

Eine Patientin mit Leukoplakie. Sowohl in Weißlicht als auch in NBI eine zystoskopische Blickdiagnose. 


\subsubsection{Alte Resektionsnarbe}

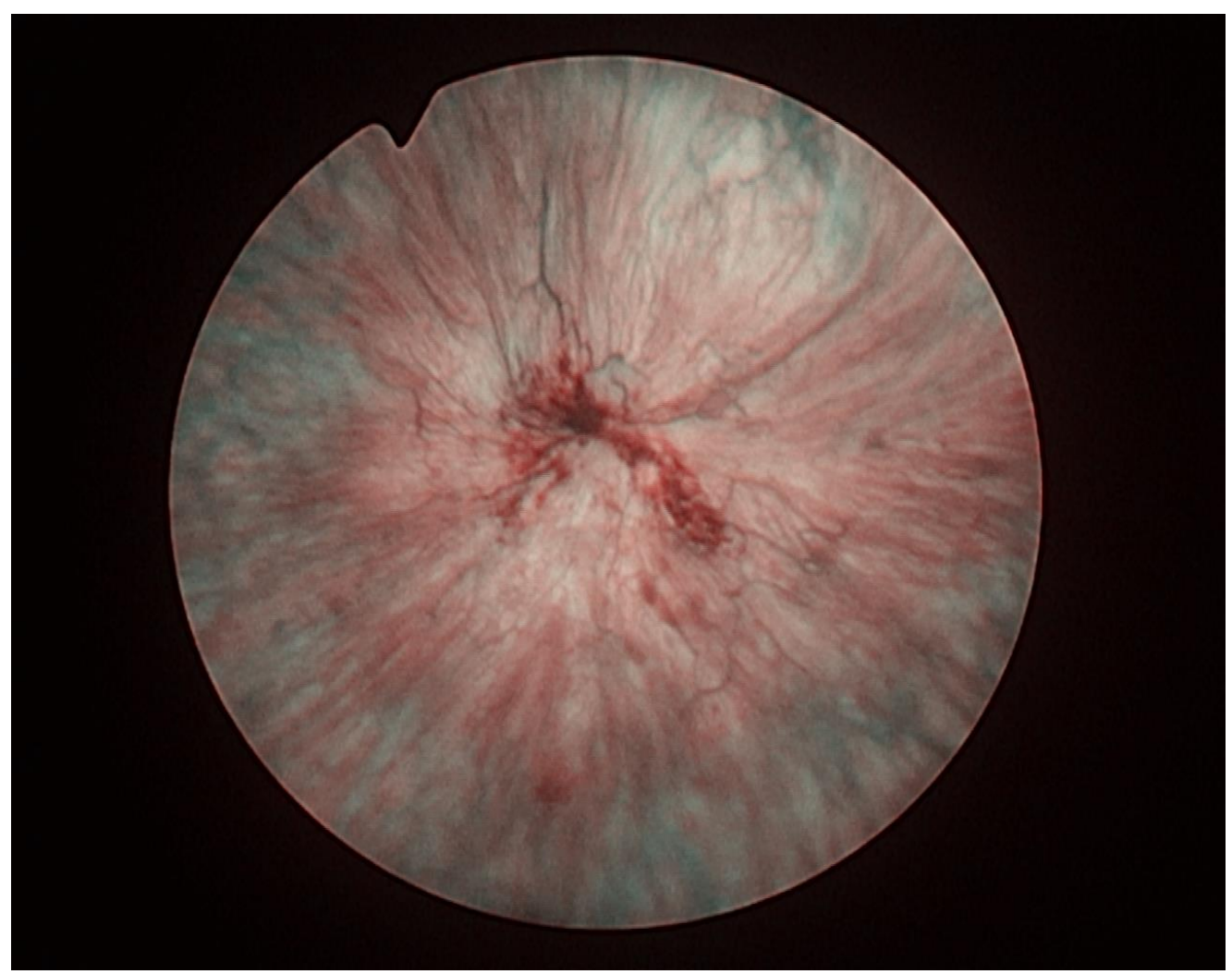

Klinisch eine zusammengezogene Narbe nach vorhergehender TUR-Blase im Sinne einer Resektionsnarbe. In NBI kann man deutlich den fehlenden Gefäßanteil von Narben erkennen. Dies ist zwar auch in Weißlicht möglich, aber von deutlich geringerem Kontrast. Histologisch liegt hier eine mittelgradige rezidivierte Urozystitis mit fokaler Urothelhyperplasie vor. 


\subsubsection{Invertiertes Papillom}



Das invertierte Papillom sieht solide aus, aber im Gegensatz zu soliden Harnblasenkarzinomen wird hier ein noch deutlicherer Übergang zur normalen Schleimhaut erkenntlich, die auch im Gegensatz zu malignen Prozessen eher feinkapillär ist und demnach eher einer Urozystitis ähnelt. Auch die Gefäßstruktur des Tumors ist hierarchisch. Im NBI-Bild findet man trotz der soliden Tumorkonfiguration mehrere Aspekte, die gegen einen wenig differenzierten invasiven Prozess sprechen. 


\section{Diskussion}

\subsection{Allgemeines}

○ Können morphologische Aspekte des Tumors schematisiert werden?

○ Können maligne Befunde sicher von nicht malignen Befunden unterschieden werden?

Ein Teilaspekt dieser Arbeit war es, als Pilotstudie zur Möglichkeit einer Schematisierung zu fungieren. Dies erlaubt keine statistischen Aussagen. Die Ergebnisse müssten in größer angelegten Studien überprüft und validiert werden.

\subsection{NBI-Morphologie von Blasenkarzinomen}

\subsubsection{Gefäßmorphologie der zuführenden Gefäße}

Die Gefäßmorphologie des Tumors und der Tumorperipherie kann nach unseren Ergebnissen mit Erfahrung typisiert werden. Werden die zuführenden Tumorgefäße betrachtet, sind Urozystitiden und teilweise auch Ta-low grade-Tumoren eher feinkapillär und retikulär strukturiert. Low grade-Tumoren zeigten zudem in der Untersuchung relativ häufig Kalibersprünge der zuführenden Gefäße. Dies entspricht den bisherigen Vorstellungen einer VEGF-Expression. Eine balkige Gefäßstruktur der Peripherie und eine nicht hierarchische Gefäßstruktur spricht dagegen für das Vorliegen eines höheren Tumorstadiums (Dennie et al. 1998). Es gelang anhand der erarbeiteten Kriterien, mit geringer Fehlerquote benigne von malignen Prozessen über die Gefäßmorphologie zu unterscheiden.

\subsubsection{Gefäßausprägung}

Die Tumormorphologie kann weiterhin Hinweise auf das Tumorstadium geben. Im low gradeStadium oder bei gutartigen Läsionen wie dem „Kathetereffekt“ liegt der Gefäßanteil unter einem Drittel der Läsion. Sie werden außen durch eine prominente glasige Schicht bedeckt. Auch höhere Tumorstadien zeigen oft eine Reduktion der Gefäßanteile. Dies aber zugunsten einer soliden Struktur. 


\subsubsection{Farbintensität}

Eine weitere typische Unterscheidung kann in der Farbintensität getroffen werden. Tief im Gewebe reflektiertes Licht lässt dortige Gefäße gedämpft bräunlich erscheinen, während Kapillaren und direkt oberflächliche alte Blutauflagerungen tiefschwarz und kontrastreich abgebildet werden. Neue Blutauflagerungen imponieren hingegen scharf rötlich. Die erwartete Reflektion der tieferen Gefäße in Cyan fand nicht statt. Vielmehr als die Farbgebung war die Lichtabsorption und damit die Kontrastschärfe bei der Bestimmung der Gefäßtiefe von Bedeutung. Die Wirkung des gefilterten Lichts auf die intestinale Schleimhaut bei der Koloskopie kann somit nicht unverändert auf das Urothel übertragen werden.

\subsubsection{NBI-Morphologie eines Cis}

\section{- Gibt es morphologische Kriterien zur Bestimmung eines Cis?}

Kriterien zur Morphologiebestimmung eines Cis konnten aufgrund der geringen Fallzahl nicht erarbeitet werden. Eine unterschiedliche Nuancierung von Grün- und Blautönen, wie in der Literatur angegeben, konnte in dieser Arbeit weder bestätigt noch widerlegt werden.

Die monozentrische Untersuchung weist darauf hin, dass eine Nichtunterlegenheit von NBI gegenüber PDD hinsichtlich der Tumordetektion und der Tumorrandbeurteilung besteht. Die Ergebnisse stimmen dabei in etwa mit denen von Jecu et al. (2014) überein, der retrospektiv 253 Patienten untersuchte und eine Detektionsrate von 95,2\% erreichte. Auch Herr und Donat (2008) konnten diese Rate in einem größeren prospektiven Vergleich (103 Patienten) bestätigen. Dies sollte in folgenden multizentrischen Studien anhand größerer Patientenzahlen validiert werden.

Wünschenswert wäre eine anschließende Schematisierung für Cis-Befunde. 


\subsection{Vergleich der drei Zystoskopietechniken: Weißlicht, PDD und NBI- Tumordetektion}

In dieser Untersuchung ergaben sich folgende bemerkenswerte Unterscheidungen je nach Art der Untersuchungsmethode:

- PDD hat eine geringere Spezifität als NBI und Weißlichtzystoskopie

- PDD zeigt eine oftmals optisch schwache Abgrenzung vom Tumor zu normalem Gewebe

- NBI erkennt deutlich Tumorgefäße und tumorversorgende Gefäße

- NBI zeigt eine optisch gute Abgrenzung von Tumoren zu normalem Gewebe

- Die Weißlichtzystoskopie hat aufgrund der routinierten Anwendung eine gute diagnostische Genauigkeit bezüglich Tumordetektion und Tumorgrenzen. Sie dient als gute Referenz für die zusätzlichen Bildgebungen

Dazu finden sich in der Literatur folgende Aussagen. Die Sensitivität der Tumordetektion ist in NBI und PDD in etwa gleich, Vorteile gegenüber einer einfachen Weißlichtzystoskopie sind wahrscheinlich und in mehreren Studien belegt (Daneshmand et al. 2014; Drejer et al. 2017; Lee et al. 2015), dementsprechend erhielten beide Bildtechniken auch Einzug (in unterschiedlichem Empfehlungsgrad) in die vorliegenden internationalen und nationalen Leitlinien. Damit werden die eigenen Ergebnisse bestätigt.

Die Aussagekraft dieser Dissertation hinsichtlich der Tumordetektion bleibt aufgrund der geringen Patientenzahl eingeschränkt. Aber auch Shadpour et al. (2016) haben anhand von 50 Patienten die signifikante Überlegenheit von NBI in der Tumordetektion (98,9\% vs. 89,4\%; $\mathrm{p}=0,001)$ beschrieben. Hier wurde die erhöhte Detektionsrate mit einer - wenn auch nicht signifikanten - Rate an falsch positiven Befunden erkauft (5,8 \% vs. 9,6\%; $\mathrm{p}=0,051)$.

- Können alle tumorsuspekten Areale, die im Weißlicht oder der PDD-Technik detektiert werden auch in der NBI-Technik gefunden werden?

- Ist die NBI-Technik in der Lage, zusätzliche Tumoren zu detektieren?

In dieser Untersuchung konnte gezeigt werden, dass

- NBI genauso viele Tumoren detektiert wie PDD. 
- NBI in der Definition der Tumorgrenzen der PDD überlegen ist

- NBI intraoperativ keine zuverlässige Unterscheidung der Infiltrationstiefe erlaubt

- eine akkurate Unterscheidung zwischen low grade- und high grade-Läsionen anhand des vorstehenden Schemas und der nachfolgenden Ausführungen erfolgen kann

- in der Nachresektion und bei Entzündungen NBI Vorteile gegenüber PDD hat

- eine Unterscheidung zwischen Urozystitis und Tumor NBI in 96,1 \% der Fälle möglich war

- Eine ausgeprägte Urozystitis verschlechtert die Beurteilung des Tumorrandes in allen bildgebenden Techniken

- die Gefäßmorphologie des Tumors und der Tumorperipherie kann nach einer kurzen Lernkurve erkannt werden

- Die Weißlichtzystoskopie als alleiniges Diagnostikum weiter ihre Berechtigung hat

- Die Weißlichtzystoskopie ist der „Goldstandard“ und zeigt ebenfalls eine hohe Sensitivität und Spezifität.

Die Studie von Ma et al. (2015a) ergab bezüglich der Tumordetektion abhängig von der verwendeten Methode eine deutliche Überlegenheit für NBI gegenüber Weißlicht $(94,5$ vs. 75,8$)$.

Ein Kritikpunkt an der Studie von Ma, Wang et al. sowie auch an Kobatake et al. (2015) ist, dass die Patienten, die in NBI reseziert wurden, vorher zusätzlich in Weißlicht zystoskopiert wurden. Die Dauer der Zystoskopie ist dadurch nahezu verdoppelt, was allein schon eine Verbesserung der Tumordetektion bewirkt.

Relevanz für den klinischen Alltag könnten diese Ergebnisse auch unter Betrachtung der Aspekte Invasivität, Komplikationen und Kosten haben. Durch zusätzliche bildgebende Techniken wie NBI konnte eine Verminderung der Rezidivrate der NMIBC, jedoch nicht der Tumorprogressionsrate erreicht werden (Ma et al. 2015b). Dies bedeutet für den Patienten letztendlich die Möglichkeit einer geringeren Traumatisierung über die Reduktion der Zahl der transurethralen Eingriffe, wahrscheinlich aber nicht ein längeres krankheitsspezifisches Überleben.

Die postoperativen, teils immanenten Folgen wie Harnröhrenstrikturen, Perforationen oder Blasenfunktionsstörungen sowie die psychologische Belastung eines Krankenhausaufenthaltes sind ebenfalls nicht zu unterschätzen. Sie treten in ihrer Häufigkeit proportional zu der Anzahl transurethraler Eingriffe auf (Yoshimura et al. 2005). 
PDD lässt keine Unterscheidung von Entzündung und Tumor zu, die Rate an falsch positiven Befunden ist gerade im Bereich von Nachresektionen $(\mathrm{p}=0,01 ; \mathrm{OR}=2,37)$ und vorangegangener intravesicaler Therapie ( $\mathrm{p}=0.03$; OR=1,78) signifikant erhöht (Draga et al. 2009); hierbei ist der Unterschied noch signifikanter, wenn die intravesicale Therapie weniger als sechs Monate zurückliegt. Ein weiterer Nachteil des PDD-Verfahrens bei der Tumordetektion ist die notwendige präoperative Einwirkzeit. Personalunterbesetzung und/oder fehlerhafte organisatorische Abläufe sowie Schmerzangaben des Patienten können gehäuft dazu führen, dass die Einwirkzeit zu kurz und somit die intraoperative Fluoreszenz zu schwach ist.

Zudem ist die Schleimhautbenetzung mit Hexaminolävulinat im Blasendivertikel reduziert, was die Beurteilung von Divertikeltumoren einschränkt.

Einige benigne Befunde sind in NBI demgegenüber mit hoher Sicherheit zu identifizieren, hierzu gehören die kontrastreichen Blutauflagerungen (100\% richtig positive) und die Leukoplakie. Auch die alte Resektionsnarbe ist aufgrund der Avaskularität in NBI sicher zu identifizieren. Eine Unterscheidung gelang aber auch in Weißlicht (100\%), systemimmanent jedoch nicht in PDD.

\subsubsection{Vorhersage der Histologie}

- Ist in der NBI-Technik eine Stadiendifferenzierung (Invasion/Grading) des Blasenkaržnoms möglich?

Die intraoperative Prädiktion des Tumorstadiums gelingt in mäßiger Genauigkeit und Zuverlässigkeit. Eine Unterscheidung der Infiltrationstiefe über eine Farbreflexion in cyan gelang nicht. Vielmehr war ein Unterschied über den Kontrast zu erkennen. Oberflächliche Kapillaren absorbieren dabei stärker und erscheinen deutlich dunkler, während tiefer gelegene Gefäße weniger Licht absorbieren, da die Eindringtiefe der niedrigen Wellenlängen nicht ausreicht (siehe: Physikalische Grundlagen).

Es kann erfahrungsabhängig mithilfe des erarbeiteten Schemas gelingen, low grade von high gradeTumoren zuverlässig zu unterscheiden; es sollte auch möglich sein, Ta-Läsionen von muskelinvasiven Tumoren zu unterscheiden. Die Rolle der T1-Tumoren ist aktuell noch schwer zu beurteilen beziehungsweise intraoperativ vorherzusagen. Dies bestätigen auch die Ergebnisse von (Hess et al. 2011), worin jedoch die perioperative Urinzytologie als nichtinvasive, aber kostspielige Variante, zusätzlich zu Hilfe genommen wurde (Ma et al. 2015a). Da insgesamt nur wenige Daten existieren, sollten sich von Untersucherseite verblindete prospektive hochvolumige 
Studien anschließen, die anhand des erarbeiteten Schemas eine intraoperative Stadieneinteilung vorhersagen. Diese Vorhersage ist mindestens von hoher Bedeutung für die Unterscheidung von benignen zu malignen Befunden. Erst bei Erreichen eines hohen Konfidenzintervalls wäre eine resect and discard-Strategie praktikabel. Der Verzicht der histologischen Befundsicherung wird jedoch auch in der Gastroenterologie kontrovers diskutiert.

Die Muskelinvasion wurde in allen vorkommenden Fällen richtig vorhergesagt. Diese Erkenntnis beruhte aber nicht auf unterschiedlichen Farbaspekten in der NBI-Technik, sondern auf dem soliden Grundaspekt der infiltrativen Tumoren (knolliges Wachstum, größerer Widerstand bei der Resektion). Es war die solide Tumorkonfiguration an sich, die eine Muskelinfiltration erahnen ließ. Somit lässt sich die Infiltrationstiefe anhand von NBI-Kriterien nicht schematisieren. Nach den Vorgaben der aktuellen Leitlinien ist die Resektion von Muskelgewebe unerlässlich. Bei der Vorhersage eines nicht muskelinvasiven Tumors ist dieser dennoch mit Muskelgewebe zu resezieren, was der vorherigen Prädiktion der Invasionstiefe lediglich einen kurzfristigen (bis zur histologischen Sicherung anhaltenden) prognostischen Wert beimisst.

Keine der bildgebenden Techniken lässt eine sichere Aussage über den Tumorgrund und die Invasionstiefe eines Tumors zu. Ein Rückschluss auf die Invasionstiefe könnte nach unserer Meinung nur über die zuführenden Gefäße erfolgen. Aus den gesammelten Erfahrungen könnte jedoch eine Tumorgrundbeurteilung bei Cis-Tumoren gelingen.

Alle anderen Tumoren müssten erst bis auf das Niveau reseziert werden, um eine Aussage zu treffen zu können. Die entstandene Resektionsspur lässt keine Beurteilung des eigentlichen Tumorgrundes zu. Wissenschaftliche Artikel über die Beurteilung des Tumorgrundes existieren nach unseren Recherchen noch nicht.

NBI erlaubte an den untersuchten Patienten eine sehr gute Histologieprädiktion. Jedoch reicht die Patientenzahl für eine statistisch untermauerte Aussage nicht aus. 


\subsection{Präzision der Ausdehnungsbestimmung des Blasenkarzinoms}

\subsubsection{Tumorrand}

- Ist eine genaue Beurteilung des Tumorrandes, also des genauen Übergangs von pathologischem zu gesundem Gewebe möglich?

In der Beurteilung des Tumorrandes ist die NBI-Darstellung der Weißlichtzystoskopie und der PDD nicht unterlegen. In 88,5 \% der Fälle konnte die Kombination der bildgebenden Techniken die genaue Tumorausdehnung korrekt vorhersagen.

Wie auch in dieser Arbeit konnten Ma et al. anhand von 150 Patienten zeigen, dass eine Reduktion der positiven Tumorränder in NBI möglich ist. Die Resektion wurde in deren Arbeit mit einem Holmium-Laser durchgeführt. In der vorliegenden Arbeit hingegen wurde die routinemäßig häufigste Methode zur Resektion, die TUR-B angewendet. Die Aussage über den Tumorrand wurde bei Ma et al. anhand eines Tumornachweises oder einer Tumorfreiheit in der Nachresektion nach drei Monaten getroffen. Besser wäre es, die Tumorränder in der Primärresektion einzusenden, mit dem Ziel, dass diese dann tumorfrei sind. Hier müsste sich eine prospektive, matched pair-Analyse anschließen. 


\subsubsection{Weiteres}

Kritisch zu bemerken ist sicherlich die unterschiedliche Bildqualität bei der PDD-Zystoskopie. Diese ist positiv abhängig von der Lichtquelle, ihrer Leistung mit idealerweise $100 \%$ und den unterschiedlichen Lichtleitkabeln, wobei ein PDD Lichtleitkabel aufgrund des vorzuschaltenden Filters naturgemäß mehr Energie leisten muss als ein „normales“ Zystoskopie-Lichtleitkabel, da der Filter einige Wellenlängen des Lichtes ,schluckt“. Zusätzlich sollte die Blase einen wenig bis mäßig gefüllten Zustand haben, da eine höhere Entfernung zum Blasengewebe bei hohen Füllungsvolumina eine Abschwächung der Lichtintensität bewirkt. Ein Hochstellen der Lichtenergie aus der Lichtquelle würde wiederum das Bild verrauscht und kontrastärmer erscheinen lassen. Die für gewöhnlich nutzungsabhängig nachlassende Qualität der verwendeten Optik über Faserbrüche sowie des Lichtleitkabels führt auch zu einer schwächeren Lichtausbeute und somit einem schlechteren Bild. Demnach wurden alle Zystoskopieoptiken vor dem Einsatz geprüft und regelmäßig gewartet.

Laut der Firma Olympus ergibt sich auch unter den entsprechend angebotenen NBI-fähigen Produkten eine unterschiedliche Qualität. So ist das Bild in der HD-Zystoskopie naturgemäß deutlich schärfer als das der normalen NBI-Zystoskopie. Dies liegt an dem jüngeren Produktionsdatum und der damit einhergehenden Verbesserung der Chips (Chip on the Tip). Insgesamt gilt es, in der Zukunft hausintern und zumindest national eine Basisqualität festzulegen und einen Standard der NBI-Zystoskopie auch über eine einfache Handhabung für ärztliches Personal und Pflegepersonal zu entwickeln.

NBI sollte zudem bei Resektionen mit einer starken Blutungsgefahr nicht verwendet werden, da die Sicht aufgrund der starken Lichtabsorption deutlich eingeschränkt ist.

Weiterhin wurde die OP-Zeit durch die Vielzahl an Kamerakopfwechseln durchaus verlängert. Im Moment ist es nur möglich, die Weißlichtzystoskopie mit einem der Verfahren zu kombinieren. Eine Kombination von PDD und NBI über ein und denselben Kamerakopf ist aufgrund der unterschiedlichen Filtertechniken noch nicht möglich. 


\subsection{Diskussion von Material und Methoden}

Die Studie wurde bewusst in verschiedene aufeinander aufbauende Abschnitte gegliedert. Zur Erfahrungssammlung und Erstellung von Kriterien, sowie auch zur abschließenden Überprüfung der optischen Kriterien genügte deshalb ein ,Convenience-Sample mit großzügigen patientenspezifischen Einschlusskriterien. Das heterogene Patientenkollektiv entspricht somit am ehesten den Gegebenheiten in der klinischen Routineversorgung.

Eine methodische Schwäche ist die monozentrische Studienanlage mit unterschiedlichen Untersuchern und damit dem Risiko von uneinheitlichen Ergebnissen. Durch die operativen Standards im Klinikum Wilhelmshaven sowie den Ausschluss jüngerer Assistenzärzte gehen wir von einem äquivalenten Ausbildungsstand hinsichtlich der operativen Fertigkeiten bei einem endoskopischen Routineeingriff aus. Der Ausschluss von Assistenzärzten in jüngeren Ausbildungsjahren erfolgte, da diese sich zuerst mit dem Handling und der Beurteilung der TURBlase in Weißlicht vertraut machen sollten. Ein Einschluss wäre möglich gewesen, hätte jedoch zu einer deutlich größeren Streuung der Befunde führen können. Das erwähnte Vorgehen dürfte o.g. methodische Schwäche einschränken. Die Erfahrung mit der NBI-Technik mussten sich alle aneignen. Hieraus resultierte selbstverständlich eine Lernkurve. Dies wurde durch die Implementierung einer präliminären Untersuchung berücksichtigt.

Die Studie hat ein hohes Risiko für einen methodisch verursachten Bias, da weder eine Verblindung stattfand noch eine simultane Untersuchung mit allen Techniken möglich ist. So erfolgte sowohl die Tumordetektion als auch die Beurteilung des Tumorrandes bei den zuletzt eingesetzten Bildtechniken unter der Kenntnis der jeweils Vorhergehenden. Ob ein Tumor, der bereits in NBI gesehen worden ist also auch tatsächlich in PDD und Weißlicht gesehen worden wäre, ist nicht $\mathrm{zu}$ sagen. Hierzu ist eine verblindete Studie mit mehreren Armen und verschiedenen festgelegten Sequenzen notwendig (siehe Tabelle 8). Auch die Tumorrandbeurteilung in Weißlicht und PDD unterliegt rein optischen Kriterien und somit einem hohen Bias.

Der Untersuchungszeitraum hätte zu einer statistisch reliabeleren Aussage deutlich verlängert werden müssen. Da es sich hier jedoch nur um eine Pilotstudie handelte, genügte die Begrenzung der Patientenzahl. Die klinischen Erfahrungen mit dem NBI-Verfahren und die sich hieraus ergebenden Tendenzen sollten als Grundlage für die Planung größer angelegter mehrarmiger und verblindeter Studien dienen und dort adaptiert und validiert werden. 
Der Vergleich der Surrogatmarker Tumorrand (R1-Status) und Tumordetektion wurde gewählt, da dies die einzigen operativen, unabhängigen Variablen sind, die simultan stark mit der Rezidivwahrscheinlichkeit korrelieren (Leitlinienprogramm Onkologie (Deutsche Krebsgesellschaft 2016).

Eine Ursache für die Häufung von technischen Problemen (bspw. nicht ausgetauschte Lichtquelle, defektes Kabel) konnte nicht eruiert werden. 


\subsection{Schlussfolgerungen}

Es konnte ein Schema zur Beurteilung der Dignität und in Einschränkungen auch zur Beurteilung der Invasivität von Blasentumoren erarbeitet werden, welches sich eng am Schema von Iwatate im gastroenterologischen Bereich orientiert und erste praktikable Ergebnisse zeigt.

Insgesamt ist das ausgearbeitete Schema leicht anzuwenden. Es kann, je nach den Ergebnissen fortlaufender Studien, modifiziert werden. Die zukünftige Angabe eines Konfidenzintervalls zur sicheren Unterscheidung zwischen benignen und malignen Befunden ist notwendig. Eine Orientierung an der Gastroenterologie mit ihrem Schema ist dabei zulässig und wünschenswert.

Bei einer größer angelegten Studie wäre eine Patientenrandomisierung sowohl der jeweiligen Reihenfolge der drei bekannten bildgebenden Techniken als auch der Operateure anzustreben (siehe Tabelle 8). Nachresektionen sollten unbedingt eingeschlossen und separat beurteilt werden. Bei signifikanten Vorteilen könnte unter Umständen eine optimale Untersuchungssequenz erarbeitet werden. Eine derart umfangreiche, vielarmige Studie ist aufgrund der Häufigkeit der TUR-B in der Klinik dennoch denkbar (vor allem bei multizentrischer Umsetzung). Falls dies gelingt, könnte in größer angelegten Untersuchungen nochmals die Möglichkeit einer Dignitätsund Tumorstadienvorhersage kontrolliert werden.

Tabelle 8: Vorgeschlagenes Schema für eine Anschlussstudie zur Beurteilung der bildgebenden Techniken und ihrer optimalen Sequenz.

\begin{tabular}{|c|c|c|c|c|c|}
\hline Gruppe 1 & Gruppe 2 & Gruppe 3 & & & \\
\hline WL allein & PDD allein & NBI allein & & & \\
\hline Gruppe 4 & Gruppe 5 & Gruppe 6 & Gruppe 7 & Gruppe 8 & Gruppe 9 \\
\hline 1. WL & 1.PDD & 1. WL & 1. NBI & 1. PDD & 1. NBI \\
\hline 2. PDD & 2. WL & 2. $\mathrm{NBI}$ & 2. WL & 2. NBI & 2. PDD \\
\hline Gruppe 10 & Gruppe 11 & Gruppe 12 & Gruppe 13 & Gruppe 14 & Gruppe 15 \\
\hline 1. WL & 1. WL & 1. PDD & 1. PDD & 1. NBI & 1. $\mathrm{NBI}$ \\
\hline 2. PDD & 2. NBI & 2. WL & 2. NBI & 2. WL & 2. PDD \\
\hline 3. NBI & 3. PDD & 3. NBI & 3. WL & 3. PDD & 3. WL \\
\hline
\end{tabular}


Es gelang, ein Schema für die Tumor- und Gefäßmorphologie zu entwickeln. Diese Studie besaß nicht den Anspruch, diese abschließend in ihrer Praktikabilität zu untersuchen.

Der diagnostische Benefit korreliert mit der Expertise des Operateurs, eine Lernkurve ist sehr wahrscheinlich. Mehrere Studien haben gezeigt, dass die NBI-Technik mit einer hohen Genauigkeit (bis zu 90 \% Übereinstimmung mit dem observierenden Experten) beispielsweise auch im Computertraining/Videotraining erlernt werden kann (Higashi et al. 2010; Ignjatovic et al. 2011; Patel et al. 2011; Raghavendra et al. 2010; Rastogi et al. 2014). Um einen nennenswerten Benefit zu haben, sollte das Schema optimiert, auf Praktikabilität geprüft und dann derart digitalisiert werden, sodass ein virtuelles Training möglich wird. Hierdurch entstünde insgesamt eine höhere Patientensicherheit. Eine daraus folgende Auswirkung auf die Anzahl von Nachresektionen ist denkbar. Ein Fortschritt war bereits die Möglichkeit der digitalen Fotodokumentation über die Kamera. Hierdurch kann die Ausbildung verbessert und die Interobservervariabilität womöglich reduziert werden.

Falls dies gelingt, könnte in größer angelegten Untersuchungen nochmals die Möglichkeit einer Dignitäts- und Tumorstadienvorhersage kontrolliert werden.

Eine genaue Bestimmung der Tiefeninfiltration und damit die Entscheidung zur Fortführung oder zum Beenden der Resektion scheint nicht mit genügender Sicherheit möglich zu sein. In der Zukunft muss man sich wahrscheinlich weiter mit der histopathologischen Aussage und einer möglichen Nachresektion begnügen. Hier hätte die Implementierung der OCT (optical coherence tomography) wahrscheinlich mehr Potential. So konnte die Infiltrationstiefe in einer kleineren Serie von Karl et al. (2010) bei 14 von 14 Patienten korrekt vorhergesagt werden.

NBI sollte nicht als konkurrierendes Verfahren zur Weißlichtzystoskopie oder zur fluoreszenzgestützten Zystoskopie gesehen werden, sondern vielmehr als einfaches additives Verfahren mit Verbesserung der Detektionsrate für Blasenkarzinome. Zudem könnte es auch als wichtiges Instrumentarium im ambulanten Bereich dienen, da dann nur die einmaligen Anschaffungskosten anfallen. Hier ist eindeutig der Vorteil der nicht notwendigen Instillation eines Photosensitizers bei vermutlich äquivalenter Detektionsrate im Vergleich zur PDD zu nennen. Ein erhöhter Sterilisationsaufwand und damit ökonomischer Mehraufwand besteht im Gegensatz zur ambulant höchst selten durchgeführten fluoreszenzgestützten Zystoskopie nicht. Hieraus ergibt sich ein Mehrwert in der Praxisanwendung. Im niedergelassenen Sektor sollte sich für NBI ein Stellenwert in der Blasenkarzinomnachsorge als synergistisches Verfahren zur Weißlichtzystoskopie ergeben.

Hierzu wäre es interessant zu wissen, inwieweit die äquivalente Detektionsrate und Tumorrandbeurteilung im ambulanten Sektor reproduzierbar sind. Eine Empfehlung in den 
Leitlinien zur Nachsorge würde eine kurze Lernkurve und hohe Anwenderfreundlichkeit bei gleichzeitig verbesserter Detektion voraussetzen.

Hierzu ist beispielsweise eine verbesserte Praktikabilität mit eventueller Reduktion der Kamerakopfwechsel notwendig. Ziel müsste es sein, auf einfachen Knopfdruck die Bildtechniken umzuschalten, um so die verbesserte Diagnostik nicht auf Kosten einer verlängerten OP-Zeit zu generieren.

Zusätzlich sollten alle optischen Systeme mittlerweile (U)HD-fähig sein. Eine deutliche Verbesserung der Diagnostik ist damit zu erwarten. Hierzu gibt es belastbare Daten von Yokoyama et al. (2012). 


\section{Zusammenfassung}

In dieser Dissertation wurde der prädiktive Wert der Gefäßmorphologie des Blasenkarzinoms in der bildgebenden Technik Narrow Band Imaging im Hinblick auf die Vorhersage der Tumorausdehnung, Infiltrationstiefe und Tumorrandausdehnung untersucht. Schlussfolgernd wurde außerdem beurteilt, ob eine sichere Identifikation zwischen malignen und benignen Prozessen möglich ist. Bei den in NBI untersuchten Patienten konnte zuverlässig eine Unterscheidung zwischen Urozystitis und Blasenkarzinom getroffen werden. Eine klare Vorhersage des Tumorstadiums scheint nicht möglich und bleibt Domäne der histologischen Untersuchung. Es kann jedoch mit zunehmender Expertise weitgehend zwischen low grade- und high grade-Tumoren unterschieden werden. Die Tumordetektion und Festlegung der Tumorgrenze gelingt in NBI zuverlässig.

Insgesamt zeigte sich, dass der sichtbare Gefäßanteil bei infiltrativen Tumoren im Gegensatz zu low grade-Karzinomen und Zystitiden geringer ist. Zudem sind die zuführenden Gefäße mit niedrigerem Tumorstadium feinkapillärer. Hierzu wurde ein Schema zur Differenzierung der Tumormorphologie und der umgebenden Gefäße entwickelt.

In der Definition der Tumorgrenzen ist NBI dem PDD-Verfahren überlegen. Insgesamt scheint aber die Kombination aller bildgebenden Techniken am sinnvollsten. 


\section{Literaturverzeichnis}

Abotaleb AA, Kandeel WS, Elmohamady B, Noureldin YA, El-Shaer W, Sebaey A (2017): Bipolar plasma kinetic enucleation of non-muscle-invasive bladder cancer: Initial experience with a novel technique. Arab J Urol 15, 355-359

Abramsson A, Lindblom P, Betsholtz C (2003): Endothelial and nonendothelial sources of PDGF-B regulate pericyte recruitment and influence vascular pattern formation in tumors. J Clin Invest $\underline{112}, 1142-1151$

Adiyat KT, Katkoori D, Soloway CT, De los Santos R, Manoharan M, Soloway MS (2010): "Complete transurethral resection of bladder tumor": are the guidelines being followed? Urology $\underline{75}, 365-367$

Al Bashir S, Yilmaz A, Gotto G, Trpkov K (2014): Long term outcome of primary urothelial papilloma: a single institution cohort. Pathology $\underline{46}, 37-40$

Apolo AB, Vogelzang NJ, Theodorescu D (2015): New and promising strategies in the management of bladder cancer. Am Soc Clin Oncol Educ Book 2015, 105-112

Avritscher EB, Cooksley CD, Grossman HB, Sabichi AL, Hamblin L, Dinney CP, Elting LS (2006): Clinical model of lifetime cost of treating bladder cancer and associated complications. Urology $\underline{68}, 549-553$

Bichler KH: Einführung. In: Bichler KH, Wilbert DM, Wechsel HW, Strohmaier WL (Hrsg.): Diagnostik und Therapie des Harnblasenkarzinoms. Band 1. Einhorn-Presse-Verlag, Reinbek 1998a, 9-13

Bichler KH, Wilbert DM, Wechsel HW, Strohmaier WL: Die Stellung der transurethralen Elektroresektion in der Behandlung des Harnblasenkarzinoms (differenzierende transurethrale Resektion). In: Bichler KH, Wechsel HW, Lahme S, Wilbert DM, Strohmaier WL (Hrsg.): Diagnostik und Therapie des Harnblasenkarzinoms. Band 1. Einhorn-Presse-Verlag, Reinbek 1998c, 82-91

Botteman MF, Pashos CL, Redaelli A, Laskin B, Hauser R (2003): The health economics of bladder cancer: a comprehensive review of the published literature. Pharmacoeconomics 21 , $1315-1330$

Brandau S, Bohle A (2001): Bladder cancer. I. Molecular and genetic basis of carcinogenesis. Eur Urol $\underline{39}$, 491-497 
Brauers A, Buettner R, Jakse G (2001): Second resection and prognosis of primary high risk superficial bladder cancer: is cystectomy often too early? J Urol $\underline{165}, 808-810$

Burger M, Stief CG, Zaak D, Stenzl A, Wieland WF, Jocham D, Otto W, Denzinger S (2009): Hexaminolevulinate is equal to 5-aminolevulinic acid concerning residual tumor and recurrence rate following photodynamic diagnostic assisted transurethral resection of bladder tumors.

Urology $\underline{74}, 1282-1286$

Burger M, Grossman HB, Droller M, Schmidbauer J, Hermann G, Dragoescu O, Ray E, Fradet Y, Karl A, Burgues JP, et al. (2013): Photodynamic diagnosis of non-muscle-invasive bladder cancer with hexaminolevulinate cystoscopy: a meta-analysis of detection and recurrence based on raw data. Eur Urol $\underline{64}$, 846-854

Campbell PA, Conrad RJ, Campbell CM, Nicol DL, Mac'Taggart P (2004): Papillary urothelial neoplasm of low malignant potential: reliability of diagnosis and outcome. BJU Int $\underline{93}, 1228-1231$

Cao Y, DePinho RA, Ernst M, Vousden K (2011): Cancer research: past, present and future. Nat Rev Cancer 11, 749-754

Cauberg EC, Kloen S, Visser M, de la Rosette JJ, Babjuk M, Soukup V, Pesl M, Duskova J, de Reijke TM (2010): Narrow band imaging cystoscopy improves the detection of non-muscleinvasive bladder cancer. Urology $\underline{76}, 658-663$

Chamie K, Litwin MS, Bassett JC, Daskivich TJ, Lai J, Hanley JM, Konety BR, Saigal CS, Project UDiA (2013): Recurrence of high-risk bladder cancer: a population-based analysis. Cancer 119 , 3219-3227

Chiu HM, Chang CY, Chen CC, Lee YC, Wu MS, Lin JT, Shun CT, Wang HP (2007): A prospective comparative study of narrow-band imaging, chromoendoscopy, and conventional colonoscopy in the diagnosis of colorectal neoplasia. Gut $\underline{56}$, 373-379

Cina SJ, Epstein JI, Endrizzi JM, Harmon WJ, Seay TM, Schoenberg MP (2001): Correlation of cystoscopic impression with histologic diagnosis of biopsy specimens of the bladder. Hum Pathol $\underline{32}, 630-637$

Daneshmand S, Schuckman AK, Bochner BH, Cookson MS, Downs TM, Gomella LG, Grossman HB, Kamat AM, Konety BR, Lee CT, et al. (2014): Hexaminolevulinate blue-light cystoscopy in non-muscle-invasive bladder cancer: review of the clinical evidence and consensus statement on appropriate use in the USA. Nat Rev Urol 11, 589-596

Dekker E, van den Broek FJ, Reitsma JB, Hardwick JC, Offerhaus GJ, van Deventer SJ, Hommes DW, Fockens P (2007): Narrow-band imaging compared with conventional colonoscopy for the detection of dysplasia in patients with longstanding ulcerative colitis. Endoscopy 39, 216-221 
Denholm SW, Conn IG, Newsam JE, Chisholm GD (1990): Morbidity following cystoscopy: comparison of flexible and rigid techniques. Br J Urol $\underline{66}, 152-154$

Dennie J, Mandeville JB, Boxerman JL, Packard SD, Rosen BR, Weisskoff RM (1998): NMR imaging of changes in vascular morphology due to tumor angiogenesis. Magn Reson Med $\underline{40}$, 793-799

Deppert W (1999): [The tumor suppressor p53: "guardian of our genomes"]. Med Monatsschr Pharm 22, 166-173

Donmez G, Sullu Y, Baris S, Yildiz L, Aydin O, Karagoz F, Kandemir B (2009): Vascular endothelial growth factor (VEGF), matrix metalloproteinase-9 (MMP-9), and thrombospondin-1 (TSP-1) expression in urothelial carcinomas. Pathol Res Pract 205, 854-857

Draga RO, Grimbergen MC, Kok ET, Jonges TN, Bosch JL (2009): Predictors of false positives in 5-aminolevulinic acid-induced photodynamic diagnosis of bladder carcinoma: identification of patient groups that may benefit most from highly specific optical diagnostics. Urology $\underline{74}, 851-$ 856

Drejer D, Beji S, Oezeke R, Nielsen AM, Hoyer S, Bjerklund Johansen TE, Lam GW, Jensen JB (2017): Comparison of White Light, Photodynamic Diagnosis, and Narrow-band Imaging in Detection of Carcinoma In Situ or Flat Dysplasia at Transurethral Resection of the Bladder: the DaBlaCa-8 Study. Urology 102, 138-142

Droller MJ (1998): Bladder cancer: state-of-the-art care. CA Cancer J Clin $\underline{48}$, 269-284

EAU, Babjuk M, Burger M, Compérat E, Gontero P, Mostofi FK, Palou J, van Rhijn B, Roupret M, Shariat SF, et al.: Non-muscle-invasive Bladder Cancer, https://uroweb.org/guideline/nonmuscle-invasive-bladder-cancer/; Zugriff am 12.10.2017

Eble JN, Sauter G, Epstein JI, Sesterhenn I: Tumours of the Urinary System. In: Eble JN, Sauter G, Epstein JI, Sesterhenn I (Hrsg.): Pathology and Genetics of Tumours of the Urinary System and Male Genital Organs. IARC Press, Lyon 2004, 89-158

Folberg R, Rummelt V, Parys-Van Ginderdeuren R, Hwang T, Woolson RF, Pe'er J, Gruman LM (1993): The prognostic value of tumor blood vessel morphology in primary uveal melanoma. Ophthalmology 100, 1389-1398

Fritsch C, Ruzicka T (2006): Fluorescence diagnosis and photodynamic therapy in dermatology from experimental state to clinic standard methods. J Environ Pathol Toxicol Oncol 25, 425-439 
Gakis G, Schilling D, Perner S, Schwentner C, Sievert KD, Stenzl A (2011): Sequential resection of malignant ureteral margins at radical cystectomy: a critical assessment of the value of frozen section analysis. World J Urol 29, 451-456

Gakis G, Efstathiou J, Lerner SP, Cookson MS, Keegan KA, Guru KA, Shipley WU, Heidenreich A, Schoenberg MP, Sagaloswky AI, et al. (2013): ICUD-EAU International Consultation on Bladder Cancer 2012: Radical cystectomy and bladder preservation for muscleinvasive urothelial carcinoma of the bladder. Eur Urol 63, 45-57

GBE-Bund.de: Gesundheitsberichterstattung des Bundes, http://www.gbe-bund.de/oowa921install/servlet/oowa/aw92/WS0100/ XWD PROC? XWD 328/7/XWD CUBE.DRILL/ X WD 356/D.946/14519; Zugriff am 01.02.2018

Gekid.de: Inzidenz des Blasenkarzinoms, http://atlas.gekid.de/Tabellen/Tabellen D.php?Method=INCIDENCE EU\&ICD10=C67\%2B D09.0\%2BD41.4\&Year from $=2011 \&$ Year to $=2014 \&$ Men $=$ on $\& W o m e n=$ on $\&$ Rates $=$ on $\&$ Cases on; Zugriff am 07.01.2018

Goessl C, Knispel HH, Miller K, Klan R (1997): Is routine excretory urography necessary at first diagnosis of bladder cancer? J Urol $\underline{157}, 480-481$

Hattori S, Iwatate M, Sano W, Hasuike N, Kosaka H, Ikumoto T, Kotaka M, Ichiyanagi A, Ebisutani C, Hisano Y, et al. (2014): Narrow-band imaging observation of colorectal lesions using NICE classification to avoid discarding significant lesions. World J Gastrointest Endosc $\underline{6}$, 600-605

Hautmann R, Huland H: Harnblasenkarzinom. In: Hautmann R, Huland H (Hrsg.): Urologie. 3., überarb. Auflage. Springer, Berlin Heidelberg 2006, 202-212

Helpap B, Kollermann J (2000): Neuerungen in der histologischen WHO-Klassifikation urothelialer Harnblasentumoren und abnormer flacher Urothellasionen. Pathologe 21, 211-217

Herr HW (2015): Randomized trial of narrow-band versus white-light cystoscopy for restaging (second-look) transurethral resection of bladder tumors. Eur Urol 67, 605-608

Herr HW, Donat SM (2008): A comparison of white-light cystoscopy and narrow-band imaging cystoscopy to detect bladder tumour recurrences. BJU Int 102, 1111-1114

Herr HW, Donat SM (2011): Reduced bladder tumour recurrence rate associated with narrowband imaging surveillance cystoscopy. BJU Int 107, 396-398 
Herranz-Amo F, Diez-Cordero JM, Verdu-Tartajo F, Bueno-Chomon G, Leal-Hernandez F, Bielsa-Carrillo A (1999): Need for intravenous urography in patients with primary transitional carcinoma of the bladder? Eur Urol $\underline{36}, 221-224$

Herrmann TR, Wolters M, Kramer MW (2017): Transurethral en bloc resection of nonmuscle invasive bladder cancer: trend or hype. Curr Opin Urol 27, 182-190

Hess J, Tschirdewahn S, Szarvas T, Rossi R, Rubben H, Vom Dorp F (2011): Urothelkarzinom der Harnblase. Kombinierte endoskopische und urinzytologische Beurteilung: Lässt es sich sicher einschätzen? Urologe A $\underline{50}, 702-705$

Higashi R, Uraoka T, Kato J, Kuwaki K, Ishikawa S, Saito Y, Matsuda T, Ikematsu H, Sano Y, Suzuki S, et al. (2010): Diagnostic accuracy of narrow-band imaging and pit pattern analysis significantly improved for less-experienced endoscopists after an expanded training program. Gastrointest Endosc 2ㅡ, 127-135

Ignjatovic A, Thomas-Gibson S, East JE, Haycock A, Bassett P, Bhandari P, Man R, Suzuki N, Saunders BP (2011): Development and validation of a training module on the use of narrowband imaging in differentiation of small adenomas from hyperplastic colorectal polyps. Gastrointest Endosc 73, 128-133

Iwatate M, Ikumoto T, Hattori S, Sano W, Sano Y, Fujimori T (2012): NBI and NBI Combined with Magnifying Colonoscopy. Diagn Ther Endosc 2012, 173269

Iwatate M, Sano Y, Hattori S, Sano W, Hasuike N, Ikumoto T, Kotaka M, Murakami Y, Hewett DG, Soetikno R, et al. (2015): The addition of high magnifying endoscopy improves rates of high confidence optical diagnosis of colorectal polyps. Endosc Int Open $\underline{3}$, E140-145

Jecu M, Geavlete B, Mulțescu R, Stănescu F, Moldoveanu C, Adou L, Ene C, Bulai C, Geavlete P (2014): NBI cystoscopy in routine urological practice - from better vision to improve therapeutic management. J Med Life 7, 282-286

Jocham D, Stepp H, Waidelich R (2008): Photodynamic diagnosis in urology: state-of-the-art. Eur Urol $\underline{53}, 1138-1148$

Karl A, Stepp H, Willmann E, Buchner A, Hocaoglu Y, Stief C, Tritschler S (2010): Optical coherence tomography for bladder cancer -- ready as a surrogate for optical biopsy? Results of a prospective mono-centre study. Eur J Med Res $\underline{15}, 131-134$

Khadra MH, Pickard RS, Charlton M, Powell PH, Neal DE (2000): A prospective analysis of 1,930 patients with hematuria to evaluate current diagnostic practice. J Urol $\underline{163}, 524-527$ 
Kobatake K, Mita K, Ohara S, Kato M (2015): Advantage of transurethral resection with narrow band imaging for non-muscle invasive bladder cancer. Oncol Lett 10, 1097-1102

Kramer MW, Rassweiler JJ, Klein J, Martov A, Baykov N, Lusuardi L, Janetschek G, Hurle R, Wolters M, Abbas M, et al. (2015): En bloc resection of urothelium carcinoma of the bladder (EBRUC): a European multicenter study to compare safety, efficacy, and outcome of laser and electrical en bloc transurethral resection of bladder tumor. World J Urol 33, 1937-1943

Kriegmair M, Baumgartner R, Stepp H, Knuechel R, Hofstetter A: Die in vivo Fluoreszenzmarkierung urothelialer Neoplasien der Harnblase. In: Bichler KH, Wilbert DM, Wechsel HW, Strohmaier WL (Hrsg.): Diagnostik und Therapie des Harnblasenkarzinoms. Band 1. Einhorn-Presse-Verlag, Reinbek 1998b, 56-70

Lamm D, Herr H, Jakse G, Kuroda M, Mostofi FK, Okajima E, Sakamoto A, Sesterhenn I, da Silva FC (1998): Updated concepts and treatment of carcinoma in situ. Urol Oncol 4, 130-138

Leal J, Luengo-Fernandez R, Sullivan R, Witjes JA (2016): Economic Burden of Bladder Cancer Across the European Union. Eur Urol 69, 438-447

Lee JY, Cho KS, Kang DH, Jung HD, Kwon JK, Oh CK, Ham WS, Choi YD (2015): A network meta-analysis of therapeutic outcomes after new image technology-assisted transurethral resection for non-muscle invasive bladder cancer: 5-aminolaevulinic acid fluorescence vs hexylaminolevulinate fluorescence vs narrow band imaging. BMC Cancer 15, 566

Leitlinienprogramm Onkologie (Deutsche Krebsgesellschaft DK, AWMF): S3-Leitlinie: Früherkennung, Diagnose, Therapie und Nachsorge des Harnblasenkarzinoms. Langversion 1.1, 2016, AWMF-Registrierungsnummer 032/038OL, http://leitlinienprogrammonkologie.de/Harnblasenkarzinom.92.0.html Zugriff am 12.10.2017

Li K, Lin T, Fan X, Duan Y, Huang J (2013): Diagnosis of narrow-band imaging in non-muscleinvasive bladder cancer: a systematic review and meta-analysis. Int J Urol $\underline{20}, 602-609$

Ma T, Li J, Jiang Z, Wang W, Shao G, Zhang L, Liu Y (2015a): [Narrow band imaging-assisted holmium laser resection reduces the residual tumor rate of primary non-muscle invasive bladder cancer: a comparison with the standard approach]. Zhonghua Yi Xue Za Zhi 모, 2775-2778

Ma T, Wang W, Jiang Z, Shao G, Guo L, Li J, Zhang L, Liu Y (2015b): [Narrow band imagingassisted holmium laser resection reduced the recurrence rate of non-muscle invasive bladder cancer: a prospective, randomized controlled study]. Zhonghua Yi Xue Za Zhi 95, 3032-3035

Manski D: Urologielehrbuch.de. ohne Verlag, Stadtbergen 2017 
Michaud DS, Spiegelman D, Clinton SK, Rimm EB, Curhan GC, Willett WC, Giovannucci EL (1999): Fluid intake and the risk of bladder cancer in men. N Engl J Med $\underline{340}$, 1390-1397

Millan-Rodriguez F, Chechile-Toniolo G, Salvador-Bayarri J, Palou J, Vicente-Rodriguez J (2000): Multivariate analysis of the prognostic factors of primary superficial bladder cancer. J Urol $\underline{163}$, 73-78

Moch H, Cubilla AL, Humphrey PA, Reuter VE, Ulbright TM (2016): The 2016 WHO Classification of Tumours of the Urinary System and Male Genital Organs-Part A: Renal, Penile, and Testicular Tumours. Eur Urol 70, 93-105

Nakai Y, Tatsumi Y, Miyake M, Anai S, Kuwada M, Onishi S, Chihara Y, Tanaka N, Hirao Y, Fujimoto K (2016): Expression of ferrochelatase has a strong correlation in protoporphyrin IX accumulation with photodynamic detection of bladder cancer. Photodiagnosis Photodyn Ther $\underline{13}$, $225-232$

Naselli A, Introini C, Timossi L, Spina B, Fontana V, Pezzi R, Germinale F, Bertolotto F, Puppo $\mathrm{P}$ (2012): A randomized prospective trial to assess the impact of transurethral resection in narrow band imaging modality on non-muscle-invasive bladder cancer recurrence. Eur Urol $\underline{61}$, 908-913

Nicolau C, Bunesch L, Peri L, Salvador R, Corral JM, Mallofre C, Sebastia C (2011): Accuracy of contrast-enhanced ultrasound in the detection of bladder cancer. Br J Radiol $\underline{84}$, 1091-1099

O'Brien T, Ray E, Chatterton K, Khan MS, Chandra A, Thomas K (2013): Prospective randomized trial of hexylaminolevulinate photodynamic-assisted transurethral resection of bladder tumour (TURBT) plus single-shot intravesical mitomycin $\mathrm{C}$ vs conventional white-light TURBT plus mitomycin $\mathrm{C}$ in newly presenting non-muscle-invasive bladder cancer. BJU Int 112 , 1096-1104

Palou J, Rodriguez-Rubio F, Huguet J, Segarra J, Ribal MJ, Alcaraz A, Villavicencio H (2005): Multivariate analysis of clinical parameters of synchronous primary superficial bladder cancer and upper urinary tract tumor. J Urol $\underline{174}, 859-861$; discussion 861

Patel P, Bryan RT, Wallace DM (2011): Emerging endoscopic and photodynamic techniques for bladder cancer detection and surveillance. ScientificWorldJournal 11, 2550-2558

Ploeg M, Aben KK, Kiemeney LA (2009): The present and future burden of urinary bladder cancer in the world. World J Urol 27, 289-293

Racioppi M, Volpe A, Falabella R, Pinto F, Sacco E, Gulino G, D'Agostino D, Bassi PF (2007): The cost of treatment and follow-up of bladder cancer in Italy. Arch Ital Urol Androl 79, 111 117 
Raghavendra M, Hewett DG, Rex DK (2010): Differentiating adenomas from hyperplastic colorectal polyps: narrow-band imaging can be learned in 20 minutes. Gastrointest Endosc $\underline{72}$, $572-576$

Rastogi A, Rao DS, Gupta N, Grisolano SW, Buckles DC, Sidorenko E, Bonino J, Matsuda T, Dekker E, Kaltenbach T, et al. (2014): Impact of a computer-based teaching module on characterization of diminutive colon polyps by using narrow-band imaging by non-experts in academic and community practice: a video-based study. Gastrointest Endosc 79, 390-398

Rink M, Babjuk M, Catto JW, Jichlinski P, Shariat SF, Stenzl A, Stepp H, Zaak D, Witjes JA (2013): Hexyl aminolevulinate-guided fluorescence cystoscopy in the diagnosis and follow-up of patients with non-muscle-invasive bladder cancer: a critical review of the current literature. Eur Urol 64, 624-638

Sarkis AS, Dalbagni G, Cordon-Cardo C, Melamed J, Zhang ZF, Sheinfeld J, Fair WR, Herr HW, Reuter VE (1994): Association of P53 nuclear overexpression and tumor progression in carcinoma in situ of the bladder. J Urol 152, 388-392

Simon J, Gschwend J: Urothelkarzinom der Harnblase. In: Schmelz H, Sparwasser C, Weidner W (Hrsg.): Facharztwissen Urologie. 2. Auflage. Springer Verlag, Heidelberg 2007, 175-202

Schultheiss D, Machtens SA, Jonas U (1999): Air cystoscopy: the history of an endoscopic technique from the late 19th century. BJU Int $\underline{83}, 571-577$

Schwaibold HE, Sivalingam S, May F, Hartung R (2006): The value of a second transurethral resection for T1 bladder cancer. BJU Int 97, 1199-1201

Shadpour P, Emami M, Haghdani S (2016): A Comparison of the Progression and Recurrence Risk Index in Non-Muscle-Invasive Bladder Tumors Detected by Narrow-Band Imaging Versus White Light Cystoscopy, Based on the EORTC Scoring System. Nephrourol Mon $\underline{8}$, e33240

Shariat SF, Karakiewicz PI, Palapattu GS, Lotan Y, Rogers CG, Amiel GE, Vazina A, Gupta A, Bastian PJ, Sagalowsky AI, et al. (2006): Outcomes of radical cystectomy for transitional cell carcinoma of the bladder: a contemporary series from the Bladder Cancer Research Consortium. J Urol 176, 2414-2422; discussion 2422

Stanisic TH, Donovan JM, Lebouton J, Graham AR (1987): 5-year experience with intravesical therapy of carcinoma in situ: an inquiry into the risks of "conservative" management. J Urol $\underline{138}$, $1158-1161$

Stein JP, Lieskovsky G, Cote R, Groshen S, Feng AC, Boyd S, Skinner E, Bochner B, Thangathurai D, Mikhail M, et al. (2001): Radical cystectomy in the treatment of invasive bladder cancer: long-term results in 1,054 patients. J Clin Oncol 19, 666-675 
Stenzl A, Burger M, Fradet Y, Mynderse LA, Soloway MS, Witjes JA, Kriegmair M, Karl A, Shen Y, Grossman HB (2010): Hexaminolevulinate guided fluorescence cystoscopy reduces recurrence in patients with nonmuscle invasive bladder cancer. J Urol 184, 1907-1913

Struck JP, Kramer MW, Merseburger AS, Hartmann A, Herrmann TRW (2017): Die En blocResektion von Blasentumoren (ERBT) - eine Bestandsaufnahme. Aktuelle Urol $\underline{48}$, 306-313

Su MY, Hsu CM, Ho YP, Chen PC, Lin CJ, Chiu CT (2006): Comparative study of conventional colonoscopy, chromoendoscopy, and narrow-band imaging systems in differential diagnosis of neoplastic and nonneoplastic colonic polyps. Am J Gastroenterol 101, 2711-2716

Sureka SK, Agarwal V, Agnihotri S, Kapoor R, Srivastava A, Mandhani A (2014): Is en-bloc transurethral resection of bladder tumor for non-muscle invasive bladder carcinoma better than conventional technique in terms of recurrence and progression?: A prospective study. Indian J Urol $\underline{30}, 144-149$

Sylvester RJ, van der Meijden AP, Oosterlinck W, Witjes JA, Bouffioux C, Denis L, Newling DW, Kurth K (2006): Predicting recurrence and progression in individual patients with stage Ta T1 bladder cancer using EORTC risk tables: a combined analysis of 2596 patients from seven EORTC trials. Eur Urol 49, 466-465; discussion 475-467

Turco P, Houssami N, Bulgaresi P, Troni GM, Galanti L, Cariaggi MP, Cifarelli P, Crocetti E, Ciatto S (2011): Is conventional urinary cytology still reliable for diagnosis of primary bladder carcinoma? Accuracy based on data linkage of a consecutive clinical series and cancer registry. Acta Cytol 푸, 193-196

Utsumi T, Iwatate M, Sano W, Sunakawa H, Hattori S, Hasuike N, Sano Y (2015): Polyp Detection, Characterization, and Management Using Narrow-Band Imaging with/without Magnification. Clin Endosc 48, 491-497

Utz DC, Hanash KA, Farrow GM (1970): The plight of the patient with carcinoma in situ of the bladder. J Urol 103, 160-164

Walker L, Liston TG, RW LL-D (1993): Does flexible cystoscopy miss more tumours than rodlens examination? Br J Urol $\underline{72}$, 449-450

Whitmore WF, Jr., Bush IM (1965): Ultraviolet cystoscopy in patients with bladder cancer. Trans Am Assoc Genitourin Surg 57, 149-155

Witjes JA, Moonen PM, van der Heijden AG (2005): Comparison of hexaminolevulinate based flexible and rigid fluorescence cystoscopy with rigid white light cystoscopy in bladder cancer: results of a prospective Phase II study. Eur Urol 47, 319-322 
Wittekind C, Meyer H: TNM Klassifikation maligner Tumoren. 7. Auflage. Willey-VCH Verlag, Weinheim 2010

Wu YP, Lin T'T, Chen SH, Xu N, Wei Y, Huang JB, Sun XL, Zheng QS, Xue XY, Li XD (2016): Comparison of the efficacy and feasibility of en bloc transurethral resection of bladder tumor versus conventional transurethral resection of bladder tumor: A meta-analysis. Medicine (Baltimore) 25, e5372

Yang LP (2014): Hexaminolevulinate blue light cystoscopy: a review of its use in the diagnosis of bladder cancer. Mol Diagn Ther $\underline{18}, 105-116$

Yeung C, Dinh T, Lee J (2014): The health economics of bladder cancer: an updated review of the published literature. Pharmacoeconomics $\underline{32}, 1093-1104$

Yokoyama A, Ichimasa K, Ishiguro T, Mori Y, Ikeda H, Hayashi T, Minami H, Hayashi S, Watanabe G, Inoue H, et al. (2012): Is it proper to use non-magnified narrow-band imaging for esophageal neoplasia screening? Japanese single-center, prospective study. Dig Endosc 24, 412418

Yoshimura K, Utsunomiya N, Ichioka K, Matsui Y, Terai A, Arai Y (2005): Impact of superficial bladder cancer and transurethral resection on general health-related quality of life: an SF-36 survey. Urology $\underline{65}, 290-294$

Yuan H, Qiu J, Liu L, Zheng S, Yang L, Liu Z, Pu C, Li J, Wei Q, Han P (2013): Therapeutic outcome of fluorescence cystoscopy guided transurethral resection in patients with non-muscle invasive bladder cancer: a meta-analysis of randomized controlled trials. PLoS One $\underline{8}$, e 74142

Yuh B, Wilson T, Bochner B, Chan K, Palou J, Stenzl A, Montorsi F, Thalmann G, Guru K, Catto JW, et al. (2015): Systematic review and cumulative analysis of oncologic and functional outcomes after robot-assisted radical cystectomy. Eur Urol 67, 402-422

Zheng C, Lv Y, Zhong Q, Wang R, Jiang Q (2012): Narrow band imaging diagnosis of bladder cancer: systematic review and meta-analysis. BJU Int $\underline{110}$, E680-687 


\section{Danksagung}

Während der Arbeit an meiner Promotion haben mich viele Menschen über einen langen Zeitraum hinweg unterstützt. Dabei möchte ich mich ganz besonders bei meinem Betreuer und Doktorvater Professor Dr. med. Hans Wechsel sowie meinem Co-Betreuer Dr. med. Dr. phil. Oliver Patschan bedanken. Trotz Zeitknappheit im klinischen Alltag unterstützen sie mich stets mit Rat und Tat, sowie viel Engagement.

Des Weiteren bedanke ich mich bei meinen Kollegen, die in den Operationen über die Bilddokumentation erst eine zufriedenstellende Anzahl an Bildmaterial bereitgestellt haben. Alle genannten Personen haben mir stets geholfen und mir mit ihrem Wissen zur Seite gestanden. 


\section{Curriculum Vitae}

Am 06. November 1986 wurde ich als dritter Sohn meines Vaters Jörg-Michael Zerrenner, Veterinäringenieur und als erster Sohn meiner Mutter Marina Zerrenner, geborene Gombert, Angestellte im öffentlichen Dienst, in Crivitz geboren. Ich wuchs mit drei Halbgeschwistern in Pampow bei Schwerin auf. Dort besuchte ich den Kindergarten und ging ab 1993 in die Grundschule. Ab 1997 besuchte ich das Gymnasium Pampow. Als Austauschschüler in den USA besuchte ich von August bis November 2003 die Central High School, Lawton, Oklahoma und von November 2003 bis Mai 2004 die Duncan High School in Duncan, Oklahoma. An der Duncan High School schloss ich 2004 mit einem Honorary Diploma ab. Anschließend kehrte ich an das Gymnasium Pampow in die Oberstufe zurück. Meine schulische Ausbildung schloss ich dort mit dem Abitur 2006 ab.

Zum Wintersemester 2006 begann ich mit dem Studium der Humanmedizin an der Universität Rostock. Im Praktischen Jahr war ich von August 2011 bis Dezember 2011 in der Inneren Medizin in den Helios Kliniken Schwerin und von Dezember 2011 bis März 2012 in der Urologie desselben Krankenhauses. Von März 2012 bis Juli 2012 war ich für das Chirurgische Tertial im Kantonsspital Winterthur, Schweiz. Im November 2012 schloss ich mein Studium an der Universität Rostock mit der Approbation ab.

Zum Januar 2013 begann ich meine Weiterbildung zum Facharzt für Urologie in Wilhelmshaven. Ab August 2018 war ich als Facharzt für Urologie in Wilhelmshaven tätig. Im Oktober 2018 wechselte ich als Facharzt an das Klinikum Lüneburg, wo ich seitdem tätig bin. Von April 2019 bis September 2019 arbeitete ich dort als Funktionsoberarzt. Hiernach ließ ich mich zuerst in einer Praxis für Urologie in Rostock anstellen. Im Januar 2020 übernahm ich dann den dortigen Kassensitz und arbeite seitdem als niedergelassener Urologe. 